\title{
Black holes and general Freudenthal transformations
}

L. Borsten, ${ }^{a}$ M.J. Duff,,$^{b, c, d}$ J.J. Fernández-Melgarejo, ${ }^{e}$ A. Marrani ${ }^{f, g}$ and

E. Torrente-Lujan ${ }^{e}$

${ }^{a}$ School of Theoretical Physics, Dublin Institute for Advanced Studies, 10 Burlington Road, Dublin 4, Ireland

${ }^{b}$ Theoretical Physics, Blackett Laboratory, Imperial College London, London SW7 2AZ, U.K.

${ }^{c}$ Mathematical Institute, University of Oxford, Andrew Wiles Building, Woodstock Road, Radcliffe Observatory Quarter, Oxford, OX2 6GG, U.K.

${ }^{d}$ Institute for Quantum Science and Engineering and Hagler Institute for Advanced Study, Texas A\&M University, College Station, TX, 7r840, U.S.A.

${ }^{e}$ Fisica Teorica, Departamento de Fúsica, Universidad de Murcia, Campus de Espinardo, E-30100 Murcia, Spain

${ }^{f}$ Museo Storico della Fisica e Centro Studi e Ricerche "Enrico Fermi", via Panisperna 89A, I-00184, Roma, Italy

${ }^{g}$ Dipartimento di Fisica e Astronomia "Galileo Galilei", Università di Padova, and INFN, sezione di Padova, via Marzolo 8, I-35131 Padova, Italy

E-mail: leron@stp.dias.ie, m.duff@imperial.ac.uk, jj.fernandezmelgarejo@um.es, alessio.marrani@pd.infn.it, torrente@cern.ch 
ABstract: We study General Freudenthal Transformations (GFT) on black hole solutions in Einstein-Maxwell-Scalar (super)gravity theories with global symmetry of type $E_{7}$. GFT can be considered as a 2-parameter, $a, b \in \mathbb{R}$, generalisation of Freudenthal duality: $x \mapsto$ $x_{F}=a x+b \tilde{x}$, where $x$ is the vector of the electromagnetic charges, an element of a Freudenthal triple system (FTS), carried by a large black hole and $\tilde{x}$ is its Freudenthal dual. These transformations leave the Bekenstein-Hawking entropy invariant up to a scalar factor given by $a^{2} \pm b^{2}$. For any $x$ there exists a one parameter subset of GFT that leave the entropy invariant, $a^{2} \pm b^{2}=1$, defining the subgroup of Freudenthal rotations. The Freudenthal plane defined by $\operatorname{span}_{\mathbb{R}}\{x, \tilde{x}\}$ is closed under GFT and is foliated by the orbits of the Freudenthal rotations. Having introduced the basic definitions and presented their properties in detail, we consider the relation of GFT to the global symmetries or U-dualities in the context of supergravity. We consider explicit examples in pure supergravity, axiondilaton theories and $N=2, D=4$ supergravities obtained from $D=5$ by dimensional reductions associated to (non-degenerate) reduced FTS's descending from cubic Jordan Algebras.

KEYwords: Supergravity Models, Black Holes, Supersymmetry and Duality

ArXiv EPrint: 1905.00038 


\section{Contents}

1 Introduction 1

2 Freudenthal triple systems: definitions and properties 4

$\begin{array}{lll}3 & \text { Freudenthal dualities and planes } & 7\end{array}$

4 The orthogonal space $\mathfrak{F}_{x}^{\perp}$ and the orthogonal plane $\mathfrak{F}_{y \perp x} \quad 10$

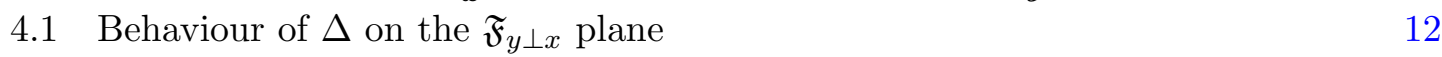

5 Freudenthal rotations: the $\Upsilon$ map and its exponential 13

$\begin{array}{lll}5.1 & \text { The exponential map on the } \mathfrak{F}_{x} \text {-plane } & 14\end{array}$

6 Pure $\mathcal{N}=2, D=4$ supergravity and degenerate FTS 16

7 The axion-dilaton $\mathcal{N}=2, D=4$ supergravity $\quad 19$

$8 \mathcal{N}=2, D=4$ supergravities from $D=5$ : the reduced $\mathfrak{F}$ case $\quad 21$

8.1 The $T^{3}$ supergravity model and $\mathfrak{F}(\mathbb{R}) \quad 23$

8.2 General $D 0-D 6 / D 2-D 4$ sectors $\quad 27$

8.3 The general $D 0-D 4$ sector $\quad 31$

8.4 F-duality preserves the $\Delta>0 \operatorname{Aut}(\mathfrak{F})$-orbits: the $S T U$ model 34

9 Linear realisations of general Freudenthal transformations $\quad 36$

$\begin{array}{lll}9.1 & \text { Anti-symplectic realisation: } \Delta<0 & 37\end{array}$

9.1.1 Anti-symplectic symmetries and parity transformations 38

9.2 Gauged symplectic realisation: $\Delta<0 \quad 38$

$\begin{array}{ll}9.3 \text { Gauged Aut }(\mathfrak{F}) \text { realisation: } \Delta<0 & 39\end{array}$

9.4 Gauged Aut $(\mathfrak{F})$ realisation: $\Delta>0 \quad 43$

9.5 Non-trivial orbit stabilizers 46

10 Summary, concluding and further remarks $\quad 47$

$\begin{array}{ll}\text { A Freudenthal triples: assorted properties } & 50\end{array}$

B More T-plane properties $\quad \mathbf{5 1}$

B.1 Complexification of real $\mathfrak{F}$ 's and $\mathfrak{T}$-planes $\quad 51$

B.2 Maximal rank $\mathfrak{T}$-planes are disjoint 53

B.3 Rank on the $\mathfrak{T} / \mathfrak{F}$-plane 54

B.4 The $\mathfrak{T}$ - or $\mathfrak{F}$-plane as a (quadratic) sub-FTS system: Euclidean and hyperbolic planes $\quad 54$

B.5 Coordinates in the $\mathfrak{T}$-plane. Orbits 56

B.6 "Light-cone" coordinates in the T-plane 57 


\section{Introduction}

Recent observations, consistent with expectations for the shadow of a Kerr black hole $(\mathrm{BH})$ as predicted by general relativity, have been, for the first time, presented $[1,2]$. This result demonstrates yet again the effectiveness of general relativity, but also serves to emphasise the need to address the long-standing puzzles presented by its $\mathrm{BH}$ solutions. A classical stationary $\mathrm{BH}$ solution is characterised by its mass $M$, angular momentum $J$ and charge $Q$ alone. In particular, its horizon area is a simple function of these three quantities. Identifying the horizon area as an entropy (determined up to a numerical constant of proportionality), the classical mechanics of BHs obeys a set of laws are directly analogous to those of thermodynamics [3-5]. Hawking's prediction [6, 7] that BHs quantum mechanically emit thermal radiation at the semiclassical level fixes the Bekenstein-Hawking area/entropy relation to be precisely (where the usual constants $c=\hbar=G=1$ ),

$$
S_{\mathrm{BH}}=\frac{A_{\text {horizon }}}{4}
$$

and suggests that the thermodynamic interpretation of $\mathrm{BH}$ mechanics is more than a mere analogy. However, it also presents an immediate question. A large BH carries a huge entropy, yet is classically characterised entirely by $M, J$ and $Q$. Where, then, are the microscopic degrees of freedom underpinning the entropy?.

Any complete theory of quantum gravity should address this challenge in some way or at least advance in this direction. String/M-theory provides an answer for a very special class of extremal dyonic BHs, where the calculations are made tractable by the presence of some preserved supersymmetries [8]. This result and its generalisations depend on a range of mathematical and theoretical insights. In particular, symmetries, duality transformations and the mathematical structures upon which they are realised, constitute important tools in the study of black hole solutions in general relativity and its supersymmetric extension, supergravity, which provides the low-energy effective field theory limit of string/M-theory. For instance, the non-compact global symmetries of supergravity theories [9], or U-dualities in the context of M-theory [10, 11], have played a particularly crucial role, starting with the work of [12]. For a large class of $\mathcal{N} \geq 2$ Poincaré supergravity theories with symmetric scalar manifolds ${ }^{1}$ the U-duality groups are of "type $E_{7}$ ", a class of groups sharing the same algebraic structure as the second largest exceptional Lie group $E_{7}$ [14]. Groups of type $E_{7}$ are axiomatically characterised by Freudenthal triple systems

\footnotetext{
${ }^{1}$ For a survey of symmetric spaces in supergravity see [13].
} 
(FTS) [14-16]. An FTS is a vector space $\mathfrak{F}$ with, in particular, a symmetric four-linear form $\Delta(x, y, z, w)$ (see section 2 for full details). The automorphism group Aut $(\mathfrak{F})$ of the FTS is the U-duality group $G_{4}$ of the associated 4 d supergravity. The electromagnetic charges carried by the static extremal black hole solutions in such theories correspond to elements $x \in \mathfrak{F}$ and fall into linear representations of the associated U-duality groups. For such theories the leading-order Bekenstein-Hawking black hole entropy is given by

$$
S_{\mathrm{BH}}=\pi \sqrt{|\Delta(x)|},
$$

where $\Delta(x):=\Delta(x, x, x, x)$ is the unique $\mathrm{U}$-duality invariant quartic polynomial of the $\mathrm{BH}$ charges.

In [17] it was shown that when the U-duality group is of type $E_{7}[14,18]$, these black hole solutions enjoy a nonlinear symmetry, named Freudenthal duality, acting on their associated charge vectors $x$. This holds for instance in all $\mathcal{N}>2$-extended, $D=4$ supergravities, as well as in all $\mathcal{N}=2$ supergravities coupled to vector multiplets with symmetric scalar manifolds. However, supersymmetry is not a necessary ingredient (e.g. in the case of $\mathfrak{F}\left(J_{3}^{\mathbb{C}_{s}}\right)$ and $\mathfrak{F}\left(J_{3}^{\mathbb{H}_{s}}\right)$; cfr. table 1$)$.

In [19] Freudenthal duality was then generalised to a symmetry not only of the Bekenstein-Hawking black hole entropy $S_{\mathrm{BH}}$, but also of the critical points of the black hole effective potential $V_{\mathrm{BH}}$ : regardless of supersymmetry, such a formulation of Freudenthal duality actually holds for any Maxwell-Einstein system coupled to a non-linear sigma model of scalar fields, in four dimensions.

The role of Freudenthal duality in the structure of extremal black hole solutions was investigated in [20], in the framework of ungauged $\mathcal{N}=8, D=4$ maximal supergravity. In particular, the most general solution to the supersymmetric stabilisation equations where shown to be given by the F-dual of a suitably defined real 56-dimensional vector, whose components are real harmonic functions in $\mathbb{R}^{3}$ transverse space. Then, in [21] Freudenthal duality was also shown to be an on-shell symmetry of the effective, one-dimensional action describing the dynamics of scalar fields in the background of a static, spherically symmetric and asymptotically flat black hole in $\mathcal{N}=2, D=4$ supergravity. In [22] it was shown that the generalised, scalar-dependent Freudenthal duality introduced in [19] actually is a symmetry of the equations of motion of the full theory, and is not restricted to the extremal black hole solutions or their effective action. Remarkably, in [22] Freudenthal duality was also applied to world-sheet actions, such as the Nambu-Goto world-sheet action in any $(t, s)$-signature spacetime, then allowing for an F-dual formulation of Gaillard-Zumino duality [23-25] on the world-sheet.

It is also here worth remarking that, in recent years, groups of type $E_{7}$, Freudenthal triple systems, and Freudenthal duality have also appeared in several indirectly related contexts, such as the relation to minimal coupling of vectors and scalars in cosmology and supergravity [26, 27], Freudenthal gauge theory (in which the scalar fields are $\mathfrak{F}$-valued) [28], multi-centered BPS black holes [29], conformal isometries [30], Hitchin functionals and entanglement in quantum information theory [31-35]. ${ }^{2}$

\footnotetext{
${ }^{2}$ Freudenthal duality in the context of entanglement and Hitchin functionals can actually be related back to its application to black holes via the black-hole/qubit correspondence [34, 36-39].
} 
Our focus here is on the notion of general Freudenthal transformations (GFT), introduced in [29]. In this work it was shown

that F-duality can be generalised to an Abelian group of transformations

$$
x \mapsto x_{F}=a x+b \tilde{x} .
$$

The GFT leave the quartic form invariant up to a scalar factor $\lambda^{2}=a^{2} \pm b^{2}$,

$$
\Delta\left(x_{F}\right)=\lambda^{4} \Delta(x)
$$

The entropy, ADM mass and, for multicenter solutions in some specific models, the intercentre distances scale as

$$
S_{B H} \rightarrow \lambda^{2} S_{B H}, \quad M_{A D M} \rightarrow \lambda M_{A D M}, \quad r_{a b} \rightarrow \lambda r_{a b},
$$

while the scalars on the horizon and at infinity are left invariant.

The properties of GFT, in particular the properties of the quartic FTS invariant (or the Bekenstein-Hawking entropy in physical terms), can be traced back to the existence and properties of Freudenthal planes in $\mathfrak{F}$. This notion first appears in the mathematical literature in [14]. Given an F-dual pair $x$ and $\tilde{x}$ we define the Freudenthal plane $\mathfrak{F}_{x} \subset \mathfrak{F}$ as the set of all elements

$$
y_{x}=a x+b \tilde{x}, \quad a, b \in \mathbb{R} .
$$

The $\mathfrak{F}_{x}$-plane is closed under GFT. From (1.4) we see that the quartic form and, thus, the Bekenstein-Hawking entropy, is invariant under the special set of GFT with $\lambda= \pm 1$. In particular, for any $x, \Delta(x) \neq 0$ there exists a one-parameter subgroup of $\operatorname{Aut}(\mathfrak{F})$ that preserves the $\mathfrak{F}_{x}$-plane and the Bekenstein-Hawking entropy. These will be referred to as Freudenthal rotations. Although GFT are non-linear, there always exists a linearly acting "gauged" U-duality ${ }^{3}$ transformation that sends $x$ to $x_{F}$.

In the present work we introduce in detail these constructions and develop their applications to black holes in supergravity, as summarised here. An extended treatment of GFT is presented in sections $2-5$. In the following sections these mathematical tools will be applied to the physics of black holes solutions in supergravity. First, in section 6 we will study the entropy properties of $\mathcal{N}=2, D=4$ pure supergravity from the point of view of the FTS formalism. This provides an example of a degenerate FTS, where the quartic invariant is a positive definite perfect square. As a consequence the Freudenthal plane in this case coincides with the entire FTS and the GFT are transitive on the space of charges. The Freudenthal rotations correspond precisely to familiar electromagnetic duality. To go beyond electromagnetic duality we consider in section 7 the axion-dilaton model, an $\mathcal{N}=2, d=4$ supergravity minimally coupled to one vector multiplet, which can be considered a consistent truncation of $\mathcal{N}=4$ supergravity. Again, this model is degenerate and cannot be uplifted to $D=5$. This is reflected in the non-reduced character of the FTS; it is not built from an underlying cubic Jordan algebra. In section 8 we proceed to the analysis of $\mathcal{N}=2, D=4$ supergravities admitting a $D=5$ origin. The

\footnotetext{
${ }^{3}$ Note, here we are considering continuous charges; when they are quantised this no longer holds.
} 
mathematical structure of these models is that of a reduced FTS, which may be derived from a cubic Jordan Algebra, $J_{3}$, so that $\mathfrak{F} \cong \mathfrak{F}\left(J_{3}\right)$. In first place we study the $T^{3}$ model, or in Freudenthal terminology $\mathfrak{F}(\mathbb{R})$.

In subsection 8.4 we study the question of orbit stratification of the $\Delta>0$ locus of $\mathfrak{F}(\mathbb{R})$ and its preservation by GFT. In section 9 we show, in different examples, how the action of GFT, and, in particular, Freudenthal duality can be realised by U-duality transformations that are "gauged" in the sense that they depend on the element of $\mathfrak{F}$ to which they are applied. Finally in section 10 we present some further physical discussion, summary and conclusions. We study the properties of asymptotically small interacting black holes. In the different appendices we present a summary of formulae used throughout the work and further technical details.

\section{Freudenthal triple systems: definitions and properties}

In 1954 Freudenthal $[15,16]$ constructed the exceptional Lie group $E_{7}$ (of dimension 133) as the automorphism group of a structure based on the smallest, non-trivial $E_{7}$ irrepr. 56, in turn related to the exceptional Jordan algebra $J_{3}^{\mathrm{O}}$ of $3 \times 3$ Hermitian octonionic matrices (also referred to as the Albert algebra) [40]. Freudenthal's aforementioned construction is often referred to as a Freudenthal triple system (FTS) for reasons that shall become clear shortly.

At the end of 60's, Meyberg [41] and Brown [14] elaborated the axioms on which the, completely symmetric, ternary structure underlying an FTS is based; in fact, the $E_{7}$ irrepr. 56 is just an example of a class of modules, characterising certain Lie groups as of groups "of type $E_{7}$ ". The role of the FTS's in $D=4$ Maxwell-Einstein (super)gravity theories was discovered later [42-44] to be related to the representation of the electric-magnetic (dyonic) charges of black hole solutions.

A FTS is defined [14] as a finite dimensional vector space $\mathfrak{F}$ over a field $\mathbb{F}^{4}$ (not of characteristic 2 or 3 ), such that:

1. $\mathfrak{F}$ possesses a non-degenerate antisymmetric bilinear form $\{x, y\}$.

2. $\mathfrak{F}$ possesses a completely symmetric four-linear form $\Delta(x, y, z, w)$ which is not identically zero. This quartic linear form induces a ternary product $T(x, y, z)$ defined on $\mathfrak{F}$ by

$$
\{T(x, y, z), w\}=2 \Delta(x, y, z, w) .
$$

3. For the ternary product $T(x, y, z)$ it is required that

$$
3\{T(x, x, y), T(y, y, y)\}=2\{x, y\} \Delta(x, y, y, y) .
$$

In our case of interest, the semi-classical supergravity limit, the physical vector of charges $x$ is to be regarded as continuous and the associated FTS is taken to be over $\mathbb{R}$ or $\mathbb{C}$.

\footnotetext{
${ }^{4}$ In the following treatment, we will consider $\mathbb{F}=\mathbb{R}$ (classical/(super)gravity level). The (quantum/Dirac-Schwinger-Zwanzinger-quantized) case (and further extensions thereof) will be investigated elsewhere. The complex case $\mathbb{F}=\mathbb{C}$ is relevant for quantum qubit entanglement applications.
} 
The automorphism group of an FTS is defined as the set of invertible $\mathbb{F}$-linear transformations preserving the quartic and quadratic forms:

$$
\operatorname{Aut}(\mathfrak{F}) \equiv\left\{\sigma \in \operatorname{Iso}_{\mathbb{F}}(\mathfrak{F}) \mid\{\sigma x, \sigma y\}=\{x, y\}, \Delta(\sigma x)=\Delta(x)\right\} .
$$

An important operation in what follows is the $\mathfrak{T}$-dual, ${ }^{5}{ }^{\prime}: \mathfrak{F} \rightarrow \mathfrak{F}$, defined by

$$
x \mapsto x^{\prime}:=T(x, x, x) \equiv T(x) .
$$

Note that, the conditions $\{\sigma x, \sigma y\}=\{x, y\}$ and $\Delta(\sigma x)=\Delta(x)$ immediately imply the homogeneity of the $\mathfrak{T}$-map

$$
T(\sigma x)=\sigma T(x) .
$$

Hence $\operatorname{Aut}(\mathfrak{F})$ is the set of automorphisms in the conventional sense.

The Lie algebra $\mathfrak{a u t}(\mathfrak{F})$ of $\operatorname{Aut}(\mathfrak{F})$ is given by

$$
\mathfrak{a u t}(\mathfrak{F})=\left\{\phi \in \operatorname{Hom}_{\mathbb{F}}(\mathfrak{F}) \mid \Delta(\phi x, x, x, x)=0,\{\phi x, y\}+\{x, \phi y\}=0, \forall x, y \in \mathfrak{F}\right\},
$$

as is easily verified [45]. The first of the conditions can be restated as $\left\{\phi x, x^{\prime}\right\}=0$.

The $\mathbb{F}$-linear map $\Upsilon_{x}: \mathfrak{F} \rightarrow \mathfrak{F}$ defined by

$$
\Upsilon_{x}(y)=3 T(x, x, y)+\{x, y\} x
$$

is in $\mathfrak{a u t}(\mathfrak{F})$. This is a direct consequence of axiom III eq. (2.6). In fact note that eq. (2.1) can be reexpressed as

$$
\left\{\Upsilon_{x}(y), y^{\prime}\right\}=0
$$

Note that, in particular,

$$
\Upsilon_{x}(x)=3 x^{\prime}
$$

but' is not in $\mathfrak{a u t}(\mathfrak{F})$.

The linear map $\Upsilon_{x}$ was introduced in this $\operatorname{Aut}(\mathfrak{F})$-covariant form in [46]. For $\Delta(x) \neq 0$ we may also define the normalized map

$$
\bar{\Upsilon}_{x} \equiv \frac{1}{3 \sqrt{|\Delta(x)|}} \Upsilon_{x}
$$

Linearizing eq. (2.6) with respect to $x$ implies that $\Upsilon_{x, y}: \mathfrak{F} \rightarrow \mathfrak{F}$ defined by

$$
\Upsilon_{x, y}(z)=6 T(x, y, z)+\{x, z\} y+\{y, z\} x
$$

is also in $\mathfrak{a u t}(\mathfrak{F})$ [47] (see also eq.11.b in [14]). In particular the following relation holds $\left\{\Upsilon_{x, y}(z), z^{\prime}\right\}=0$. We have also $\Upsilon_{x, x}(z)=2 \Upsilon_{x}(z)$ and, (see [14]), for any $y, z, \Upsilon_{y, y^{\prime}}(z)=0$

Following [46] an FTS element may be assigned a manifestly Aut(F) invariant rank, an integer function between 1 and 4 defined by the relations:

$$
\begin{aligned}
& \operatorname{Rank}(x)=1 \Leftrightarrow \Upsilon_{x}(y)=0 \forall y, x \neq 0 ; \\
& \operatorname{Rank}(x)=2 \Leftrightarrow T(x)=0, \exists y \text { s.t. } \Upsilon_{x}(y) \neq 0 ; \\
& \operatorname{Rank}(x)=3 \Leftrightarrow \Delta(x)=0, T(x) \neq 0 ; \\
& \operatorname{Rank}(x)=4 \Leftrightarrow \Delta(x) \neq 0 .
\end{aligned}
$$

\footnotetext{
${ }^{5}$ Not to be confused with T-duality in string theory.
} 
The Rank 1 conditions appeared before in [15]. We define the sets of elements of a given rank $\mathfrak{F}_{(k)} \equiv\{x \in \mathfrak{F} \mid \operatorname{Rank}(x)=k\}$. The rank of a element can be related to the degree of supersymmetry preserved by the solution (see [48] and references therein).

Supergravity and the classification of FTS: an outline. An FTS is said to be reduced if it contains a strictly regular element: $\exists u \in \mathfrak{F}$ such that $T(u)=0$ and $u \in$ Range $L_{u, u}$ where $L_{x, y}: \mathfrak{F} \rightarrow \mathfrak{F} ; \quad L_{x, y}(z) \equiv T(x, y, z)$. It can be proved $[14,49]$ that every simple reduced FTS $\mathfrak{F}$ is isomorphic to an FTS $\mathfrak{F}\left(J_{3}\right)$, where

$$
\mathfrak{F}\left(J_{3}\right) \equiv \mathbb{F} \oplus \mathbb{F} \oplus J_{3} \oplus \bar{J}_{3},
$$

with $J_{3}$ denoting a rank-3 Jordan algebra. All algebraic structures in $\mathfrak{F}\left(J_{3}\right)$ can be defined in terms of the basic Jordan algebra operations [14, 49] (also cfr. [50] and refs. therein). In a Maxwell-Einstein physical framework, the presence of an underlying Jordan algebra $J_{3}$ corresponds to the fact that the $D=4$ Maxwell-Einstein (super)gravity theories can be obtained by dimensional reduction of a $D=5$ theory, whose electric-magnetic (U-)duality ${ }^{6}$ is nothing but the reduced structure group of $J_{3}$ itself.

For $\mathfrak{F}\left(J_{3}^{\mathbb{A}}\right)$, the automorphism group has a two element centre, and its quotient yields the simple groups listed e.g. in table 1 of [22], whereas for $\mathfrak{F}\left(\mathbb{R} \oplus \Gamma_{m, n}\right)$ one obtains the semi-simple groups $\mathrm{SL}(2, \mathbb{R}) \times \mathrm{SO}(m+1, n+1)[14,46,54]$. In all cases, $\mathfrak{F}$ fits into a symplectic representation of $\operatorname{Aut}(\mathfrak{F})$, with dimensions listed e.g. in the rightmost column of table 1 of [22].

By confining ourselves to reduced FTS's $\mathfrak{F}\left(J_{3}\right)$ related to simple or semi-simple rank-3 Jordan algebras $J_{3}$, one can exploit the Jordan-Von Neumann-Wigner classification [40], and enumerate the possible FTS's, depending on their dimension $\operatorname{dim} \mathfrak{F}=2 N .^{7}$ A summary of this classification is presented in table 1.

Various $D=4$ supergravities are listed in table 1: the semi-simple cases $\mathfrak{F}\left(\mathbb{R} \oplus \Gamma_{1, n-1}\right)$ and $\mathfrak{F}\left(\mathbb{R} \oplus \Gamma_{5, n-1}\right)$ correspond to $\mathcal{N}=2$ resp. 4 Maxwell-Einstein supergravity, while $\mathfrak{F}\left(J_{3}^{\mathbb{A}}\right) \equiv \mathfrak{F}^{\mathbb{A}}$ correspond to the so-called $\mathcal{N}=2$ "magic" Maxwell-Einstein supergravities $^{8}[42]$. Moreover, $\mathfrak{F}^{\mathbb{O}_{s}} \equiv \mathfrak{F}\left(J_{3}^{\mathrm{O}_{s}}\right)$ pertains to maximal $\mathcal{N}=8$ supergravity, and the simplest reduced FTS is $\mathfrak{F}(\mathbb{R})$, related to the so-called $T^{3}$ model of $\mathcal{N}=2, D=4$ supergravity (treated in subsection 8.1).

As evident from table 1 , there are two (for $\mathbb{A}=\mathbb{R}$ ) or three (for $\mathbb{A}=\mathbb{C}, \mathbb{H}, \mathbb{O}$ ) possible FTS structures for $N=3 q+4$, where $q=\operatorname{dim}(\mathbb{R}, \mathbb{C}, \mathbb{H}, \mathbb{O})=1,2,4,8$, corresponding to $\mathfrak{F}^{\mathbb{A}}[42,43]$ and $\mathfrak{F}^{\mathbb{A}_{s}}[51,52,57]$.

Finally, an FTS is said to be degenerate if its quartic form is identically proportional to the square of a quadratic polynomial. Note that FTS on "degenerate" groups of type $E_{7}$ (as

\footnotetext{
${ }^{6}$ Here U-duality is referred to as the "continuous" symmetries of [51, 52]. Their discrete versions are the U-duality non-perturbative string theory symmetries introduced by Hull and Townsend [53].

${ }^{7}$ Reduced FTS's have at least dimension $2 N=4$, namely they contain at least $N=2$ Abelian vectors in $D=4$. Within the $\mathcal{N}=2$ interpretation, they are the $5 D \rightarrow 4 D$ Kaluza-Klein $(\mathrm{KK})$ vector (aka the $D=4$ graviphoton) and the $D=5$ graviphoton (which becomes a matter photon in $D=4$ ).

${ }^{8}$ The theories based on Lorentzian cubic Jordan algebras $J_{2,1}^{\mathrm{A}}$ and $J_{2,1}^{\mathrm{A}_{s}}$ correspond to certain classes of $\mathcal{N}=2$ supergravities with non-homogeneous vector multiplets' scalar manifolds (cfr. e.g. [55, 56]).
} 


\begin{tabular}{|c|c|c|}
\hline$N$ & $J_{3}$ & $D=4$ Maxwell-Einstein theory \\
\hline 2 & $\mathbb{R}$ & $\mathcal{N}=2 T^{3}$ \\
\hline 3 & $\mathbb{R} \oplus \mathbb{R}$ & $\mathcal{N}=2, S T^{2}$ \\
\hline 4 & $\mathbb{R} \oplus \mathbb{R} \oplus \mathbb{R}$ & $\mathcal{N}=2, S T U$ \\
\hline $5-6$ & $\mathbb{R} \oplus \Gamma_{n, 3-n}, \quad \mathbb{R} \oplus \Gamma_{n, 4-n}$ & $n=1: \mathcal{N}=2, n_{V}=4,5$ \\
\hline 7 & $\left\{\begin{array}{l}\mathbb{R} \oplus \Gamma_{n, 5-n} \\
J_{3}^{\mathbb{R}}\end{array}\right.$ & $\left\{\begin{array}{l}n=1: \mathcal{N}=2, n_{V}=5, n=5: \mathcal{N}=4, n_{V}=1 \\
\mathcal{N}=2 \text { magic } \mathbb{R}\end{array}\right.$ \\
\hline 8-9 & $\begin{array}{l}\mathbb{R} \oplus \Gamma_{n, 6-n}, \mathbb{R} \oplus \Gamma_{n, 7-n} \\
\left\{\begin{array}{l}\mathbb{R} \oplus \Gamma_{n, 8-n} \\
J_{3}^{\mathbb{C}}, \quad J_{3}^{\mathrm{C}_{s}}\end{array}\right.\end{array}$ & $\begin{array}{l}n=1: \mathcal{N}=2, n_{V}=7,8, n=5: \mathcal{N}=4, n_{V}=2,3 \\
\left\{\begin{array}{l}n=1: \mathcal{N}=2, n_{V}=9, n=5: \mathcal{N}=4, n_{V}=3 \\
\mathcal{N}=2 \text { magic } \mathbb{C}, \mathcal{N}=0 \text { magic } \mathbb{C}_{s}\end{array}\right.\end{array}$ \\
\hline $11-15$ & $\begin{array}{l}\mathbb{R} \oplus \Gamma_{n, 9-n}, \ldots, \mathbb{R} \oplus \Gamma_{n, 13-n} \\
\left\{\begin{array}{l}\mathbb{R} \oplus \Gamma_{n, 14-n} \\
J_{3}^{\mathbb{H}}, \quad J_{3}^{\mathbb{H}_{s}}\end{array}\right.\end{array}$ & $\begin{array}{l}n=1: \mathcal{N}=2, n_{V}=10-14, n=5: \mathcal{N}=4, n_{V}=5-9 \\
\left\{\begin{array}{l}n=1: \mathcal{N}=2, n_{V}=15, n=5: \mathcal{N}=4, n_{V}=10 \\
\mathcal{N}=2 \text { magic } \mathbb{H}, \quad \mathcal{N}=0 \text { magic } \mathbb{H}_{s}\end{array}\right.\end{array}$ \\
\hline $17-27$ & $\mathbb{R} \oplus \Gamma_{n, 15-n}, \ldots, \quad \mathbb{R} \oplus \Gamma_{n, 25-n}$ & $n=1: \mathcal{N}=2, n_{V}=16-26, n=5: \mathcal{N}=4, n_{V}=11-21$ \\
\hline 28 & $\left\{\begin{array}{l}\mathbb{R} \oplus \Gamma_{n, 26-n} \\
J_{3}^{\mathrm{O}}, \quad J_{3}^{\mathrm{O}_{s}}\end{array}\right.$ & $\left\{\begin{array}{l}n=1: \mathcal{N}=2, n_{V}=27, n=5: \mathcal{N}=4, n_{V}=22 \\
\mathcal{N}=2 \text { magic } \mathbb{O}, \quad \mathcal{N}=8\end{array}\right.$ \\
\hline$>28$ & $\mathbb{R} \oplus \Gamma_{n, N-2-n}$ & $n=1: \mathcal{N}=2, n_{V}>27, n=5: \mathcal{N}=4, n_{V}>22$ \\
\hline
\end{tabular}

Table 1. Classification of Freudenthal triple systems (see text for explanation).

defined in [27], and refs. therein) are not reduced and hence cannot be written as $\mathfrak{F}\left(J_{3}\right)$; they correspond to theories which cannot be uplifted to $D=5$ dimensions consistently reflecting the lack of an underlying rank-3 Jordan algebra $J_{3}$.

\section{$3 \quad$ Freudenthal dualities and planes}

F-duality. We have defined already the transformation $x^{\prime}=T(x)$, valid for a vector of any rank (see eq. (2.3)). For rank-4 charge vectors $x \in \mathfrak{F}_{(4)}$, the black hole charge Freudenthal duality is defined by $(\epsilon \equiv \epsilon(x) \equiv \operatorname{sgn} \Delta(x))$

$$
\sim: \mathfrak{F}_{(4)} \rightarrow \mathfrak{F}_{(4)}, \quad x \mapsto \tilde{x} \equiv \epsilon(x) \frac{T(x)}{\sqrt{|\Delta(x)|}}
$$

The Freudenthal duality has the following elementary properties [48]:

- It preserves the quartic norm $\Delta(\tilde{x})=\Delta(x)$; obviously $\Delta(\tilde{x}, x, x, x)=\left\{\tilde{x}, x^{\prime}\right\}=0$.

- It is an anti-involution: $\tilde{\tilde{x}}=-x$;

- It is not a U-duality, since it is non-linear and generically $\{\tilde{x}, \tilde{y}\} \neq\{x, y\}$. Also, in general, $\{\tilde{x}, y\}+\{x, \tilde{y}\} \neq 0$.

Note that, although the map $x \rightarrow \tilde{x}$ is not a U-duality, the map $\Upsilon_{x}$ (or $\bar{\Upsilon}$ ), for $x$ fixed, is indeed: 

a) linear,
b) $\Upsilon_{x} \in \mathfrak{a u t}(\mathfrak{F})$ and, finally
c) $\bar{\Upsilon}_{x}(x)=\epsilon(x) \tilde{x}$.

It follows from (c), that for $\Delta(x)>0$ the Freudenthal map $x \mapsto \tilde{x}$ can be considered as an " $x$-dependent" U-duality.

The $\mathfrak{T}$ - and $\mathfrak{F}$-planes: definition and general properties. For a general element in $\mathfrak{F}$, respectively an element $x \in \mathfrak{F}_{(4)}$, we define the associated $\mathfrak{T}$ - and $\mathfrak{F}$-planes, respectively denoted $\mathfrak{T}_{x}, \mathfrak{F}_{x}$, as the $\mathbb{R}$-linear spans of $x, x^{\prime}$ or $x, \tilde{x}$. In each case:

$$
\begin{aligned}
& \mathfrak{T}_{x} \equiv\left\{y \in \mathfrak{F} \mid y=a x+b x^{\prime}, a, b \in \mathbb{R}\right\}, \\
& \mathfrak{F}_{x} \equiv\left\{y \in \mathfrak{F}_{(4)} \mid y=a x+b \tilde{x}, a, b \in \mathbb{R}\right\} .
\end{aligned}
$$

Naturally the $\mathfrak{F}$-plane $\mathfrak{F}_{x}$ is only defined as long as $\Delta(x) \neq 0$ (maximal rank elements), while $\mathfrak{T}_{x}$ is defined for any $x$, although it degenerates to a $\mathfrak{T}$-line for rank $x<3$ elements. If they both exist, $\mathfrak{T}_{x}$ and $\mathfrak{F}_{x}$ are the same space. It is advantageous to study the properties of the $\mathfrak{T}$-planes, and when needed, to specialise to $\mathfrak{F}$-planes. We will follow this strategy in what follows.

Linearity of $\mathfrak{T}$-transformations on the $\mathfrak{T}$-plane. We first show the linearity of the $\mathfrak{T}$-dual on the $\mathfrak{T}$-plane: $\mathfrak{T}$-planes are closed under $\mathfrak{T}$-transformations. For any linear combination, one has, because of the multi-linearity of $T$, ( $a, b$ constants, $\Delta_{x}=\Delta(x)$ ),

$$
\begin{aligned}
T\left(a x+b x^{\prime}\right) & =a^{3} T(x)+b^{3} T\left(x^{\prime}\right)+3 a^{2} b T\left(x, x, x^{\prime}\right)+3 a b^{2} T\left(x, x^{\prime}, x^{\prime}\right) \\
& =a^{3} x^{\prime}+a b^{2} \Delta_{x} x^{\prime}-b^{3} \Delta_{x}^{2} x-a^{2} b \Delta_{x} x \\
& =\left(a^{2}+b^{2} \Delta_{x}\right)\left(-b \Delta_{x} x+a x^{\prime}\right)
\end{aligned}
$$

Where we have used the properties ([14], lemma 11.(abcf)):

$$
\begin{aligned}
T\left(x, x, x^{\prime}\right) & =-1 / 3 \Delta_{x} x, \\
T\left(x, x^{\prime}, x^{\prime}\right) & =1 / 3 \Delta_{x} x^{\prime}, \\
T\left(x^{\prime}, x^{\prime}, x^{\prime}\right) & =-\Delta_{x}^{2} x .
\end{aligned}
$$

We can see that eq. (3.4) is equivalent to, or simply summarizes, the relations eqs. (3.5)-(3.7).

Using eq. (3.4) we can compute the map $\Delta$ for any element on the T-plane. After a short explicit computation we have (using $2 \Delta(x)=\left\{x^{\prime}, x\right\}$ )

$$
\begin{aligned}
\Delta\left(a x+b x^{\prime}\right) & =\frac{1}{2}\left\{T\left(a x+b x^{\prime}\right), a x+b x^{\prime}\right\} \\
& =\left(a^{2}+b^{2} \Delta(x)\right)^{2} \Delta(x) .
\end{aligned}
$$

The sign of $\Delta$ on the $\mathfrak{T}$-plane is constant. Hence, in any $\mathfrak{F}$-plane, there is an element $y \in \mathfrak{F}_{x}$ such $\Delta(y)=0$ if and only if $\Delta(x)$ is negative. 
Similarly, we have the following expressions describing the behaviour of the map $\Upsilon$ on the T-plane:

$$
\begin{aligned}
\Upsilon_{x}\left(x^{\prime}\right) & =3 T\left(x, x, x^{\prime}\right)+\left\{x, x^{\prime}\right\} x=-\Delta_{x} x-2 \Delta_{x} x=-3 \Delta_{x} x, \\
\Upsilon_{x}\left(a x+b x^{\prime}\right) & =-3 b \Delta(x) x+3 a x^{\prime}=3 T\left(a x+b x^{\prime}\right) /\left(a^{2}+b^{2} \Delta_{x}\right) .
\end{aligned}
$$

Further mathematical properties of the $\mathfrak{T}$-planes are shown in section A and section B.

$\mathfrak{T}$ - and $\mathfrak{F}$-transformations on the planes. For $\tilde{x}$ well-defined, we rewrite eq. (3.4), eq. (3.9), by a redefinition of the parameters $a, b$ in terms of the F-dual eq. (3.1) as $(\epsilon=$ $\operatorname{sgn} \Delta(x))$

$$
\begin{aligned}
T(a x+b \tilde{x}) & =\epsilon\left(a^{2}+\epsilon b^{2}\right) \sqrt{|\Delta(x)|}(-\epsilon b x+a \tilde{x}), \\
\Delta(a x+b \tilde{x}) & =\left(a^{2}+\epsilon b^{2}\right)^{2} \Delta(x) .
\end{aligned}
$$

It is clear from this last expression that $a^{2}+\epsilon b^{2}= \pm 1$ defines a subset of elements in $\mathfrak{F}_{x}$ with fixed entropy: an $\mathrm{SO}(2)$ or $\mathrm{SO}(1,1)$ symmetry. Moreover, $\operatorname{sgn} \Delta(x)=\operatorname{sgn} \Delta(a x+b \tilde{x})=\epsilon$, unless $a^{2}+\epsilon b^{2}=0$. In particular

$$
\Delta(x \pm \tilde{x})=(1+\epsilon)^{2} \Delta(x) .
$$

As consequence of eq. (3.12) and eq. (3.13), the F-dual of a linear combination is given by $(\Delta(x) \neq 0, \Delta(a x+b \tilde{x}) \neq 0, \operatorname{sgn} \Delta(x)=\operatorname{sgn} \Delta(a x+b \tilde{x})=\epsilon)$

$$
\widetilde{a x+b \tilde{x}}=\frac{\epsilon T(a x+b \tilde{x})}{\sqrt{|\Delta(a x+b \tilde{x})|}}
$$

and, finally,

$$
\widetilde{a x+b \tilde{x}}=\eta(-\epsilon b x+a \tilde{x})= \begin{cases}-b x+a \tilde{x}, & (\Delta(x)>0), \\ \eta(b x+a \tilde{x}), & (\Delta(x)<0),\end{cases}
$$

where $\epsilon=\operatorname{sgn} \Delta(x), \eta=\operatorname{sgn}\left(a^{2}+b^{2} \epsilon\right)$. Clearly, if $\epsilon=1$ then $\eta=1$

As discussed in appendix subsection B.4 the $\mathfrak{F}$-plane is a, quadratic, two dimensional, sub-FTS system with suitably restricted operations $\Delta_{\mathfrak{F}}, T_{\mathfrak{T}},\{,\}_{\mathfrak{T}}$ and $I_{2}$.

On the full FTS, for maximal rank elements, one can define an (Aut( $\mathfrak{F})$-invariant) "metric" by the (non quadratic) expression

$$
(u, v) \equiv \frac{1}{4}[\{\tilde{u}, v\}+\{\tilde{v}, u\}] .
$$

and a "pseudo-norm" by

$$
\|u\|=(u, u)=\frac{1}{2}\{\tilde{u}, u\}=\epsilon \sqrt{|\Delta(u)|} .
$$


If we fix $x$, and restrict ourselves to the $\mathfrak{F}_{x}$ plane we can use the expressions in subsection B.4 (see also further properties in ref. [58]) and connect $\|u\|$ with $I_{2}(u)$ :

$$
\begin{aligned}
(u, v) & \equiv \frac{1}{4}[\{\tilde{u}, v\}+\{\tilde{v}, u\}] \\
& =\frac{1}{4}[\eta(u)\{\hat{S} u, v\}+\eta(v)\{\hat{S} v, u\}] \\
& =\frac{1}{4}(\eta(u)+\eta(v))\{\hat{S} v, u\} \\
& =\frac{1}{4} \epsilon(\eta(u)+\eta(v)) I_{2}(v, u)
\end{aligned}
$$

where $\epsilon=\operatorname{sgn} \Delta(x), \eta(x)=\operatorname{sgn} I_{2}(x)$ and $\hat{S}$ is a linear map given in section B. In particular

$$
\|u\|=\frac{1}{2} \epsilon \eta(u) I_{2}(u)=\frac{1}{2} \epsilon\left|I_{2}(u)\right|
$$

We arrive at the same conclusions as for the $\mathfrak{T}$-plane. For $\epsilon=1$, the pseudo-norm $(\cdot, \cdot)$ (or $I_{2}(u)$ ) is positive definite and the norm-preserving group is $\mathrm{SO}(2)$; thus, the $\mathfrak{F}$-plane $\mathfrak{F}_{x}$ undergoes a "spherical foliation". On the other hand, for $\epsilon=-1$, the norm is positive semi-definite and the norm-preserving group is $\mathrm{SO}(1,1)$; thus, the $\mathfrak{F}$-plane $\mathfrak{F}_{x}$ undergoes an "hyperboloid-like foliation". While the norm is timelike or null, the vector $u$ can be timelike, spacelike and null according to $\eta(x)$, the sign of $I_{2}(x)$.

As for the T-operation, the F-duality $x \rightarrow \tilde{x}$, change the character of the vector. The vectors $x, \tilde{x}$ are " $I_{2}$-orthogonal", by eq. (B.29), $(x, \tilde{x})=I_{2}(x, \tilde{x})=0 . \tilde{x}$ is timelike (resp. spacelike) if $x$ is spacelike (resp. timelike):

$$
\begin{aligned}
x \text { :lightlike } & \longleftrightarrow \tilde{x}: \text { lightlike }, \quad \tilde{x}= \pm x, \\
x: \text { timelike }(\text { spacelike }) & \longleftrightarrow \tilde{x}: \text { spacelike (timelike) }
\end{aligned}
$$

It is noted that, although the metric $I_{2}(x, y)$ is defined only inside a concrete $\mathfrak{F}$-plane, the character null, time or spacelike of a vector is an intrinsic property, as any given element belongs to one and only one $\mathfrak{F}$-plane, "its" plane, from the disjointness of the $\mathfrak{F}$-planes (see subsection B.2).

\section{The orthogonal space $\mathfrak{F}_{x}^{\perp}$ and the orthogonal plane $\mathfrak{F}_{y \perp x}$}

In general $\{x, \tilde{x}\}=-2 \sqrt{|\Delta(x)|} \neq 0$. The bilinear form $\{\cdot, \cdot\}$ is non-degenerate on $\mathfrak{F}_{x}$ by construction, since $x$ is neccesarily of maximal rank $(\Delta(x) \neq 0)$. Consequently, for a given $x \in \mathfrak{F}_{(4)}$, the FTS $\mathfrak{F}$ may be decomposed as

$$
\mathfrak{F}=\mathfrak{F}_{x} \oplus \mathfrak{F}_{x}^{\perp},
$$

where $\mathfrak{F}_{x}$ is the 2 -dimensional $\mathfrak{F}$-plane and $\mathfrak{F}_{x}^{\perp}$ is its $\left(\operatorname{dim}_{\mathbb{F}} \mathfrak{F}-2\right)$-dimensional orthogonal complement w.r.t. the bilinear form $\{\cdot, \cdot\}$ :

$$
\mathfrak{F}_{x}^{\perp}=\left\{y \in \mathfrak{F}:\{x, y\}=\left\{x^{\prime}, y\right\}=0\right\}
$$


Hence, for a given $x \in \mathfrak{F}$, any element $y \in \mathfrak{F}$ enjoys the decomposition

$$
y=y_{\| x}+y_{\perp x},
$$

where $y_{\| x} \in \mathfrak{F}_{x}$ and $y_{\perp x} \in \mathfrak{F}_{x}^{\perp}$. Note that $y_{\| x}, y_{\perp x}$ (also denoted $y_{\|}, y_{\perp}$ if there is no risk of confusion) are uniquely determined by $x$. The coordinates of $y_{\| x}=a x+b \tilde{x}$ are uniquely determined by the expressions,

$$
\begin{aligned}
& \left\{x, y_{\perp}\right\}=0 \Leftrightarrow b=\frac{-1}{2 \sqrt{|\Delta(x)|}}\{x, y\} \\
& \left\{\tilde{x}, y_{\perp}\right\}=0 \Leftrightarrow a=\frac{1}{2 \sqrt{|\Delta(x)|}}\{\tilde{x}, y\} .
\end{aligned}
$$

Or, in compact notation (with respect a fixed element $x$ ),

$$
\begin{aligned}
y_{\|} & =\frac{1}{2 \sqrt{ }|\Delta(x)|}\left|\begin{array}{cc}
x & \tilde{x} \\
\{x, y\} & \{\tilde{x}, y\}
\end{array}\right|, \\
& =\frac{1}{\left\{x^{\prime}, x\right\}}\left|\begin{array}{cc}
x & x^{\prime} \\
\{x, y\} & \left\{x^{\prime}, y\right\}
\end{array}\right| \\
y_{\perp} & =y-y_{\|} .
\end{aligned}
$$

The following properties hold (for the parallel component with respect a fixed $x$ ):

$$
\begin{aligned}
\Delta\left(y_{\|}\right) & =\frac{1}{16 \Delta(x)}\left(\epsilon\{\tilde{x}, y\}^{2}+\{x, y\}^{2}\right)^{2} \\
(a y+b \tilde{y})_{\|} & =a y_{\|}+b(\tilde{y})_{\|} \\
\widetilde{\left(y_{\|}\right)} & =\frac{\eta \epsilon}{2 \sqrt{ }|\Delta(x)|}(\epsilon\{\tilde{x}, y\} \tilde{x}+\{x, y\} x) .
\end{aligned}
$$

with $\eta=\operatorname{sgn}\left(\epsilon\{\tilde{x}, y\}^{2}+\{x, y\}^{2}\right)$. In particular, note the distributivity of $\|$ (second expression).

Note, however that

$$
\widetilde{y}_{\|} \neq(\tilde{y})_{\|}=\frac{(\{\tilde{x}, \tilde{y}\} x-\{x, \tilde{y}\} \tilde{x})}{2 \sqrt{\left|\Delta_{x}\right|}} .
$$

Obviously, a similar construction can be performed for the $\mathfrak{T}$-plane (see [14], from pg. 89 on, where such a space is used to build a Jordan Algebra for reduced FTSs).

This decomposition into "parallel" and "orthogonal" spaces and the further decomposition of the orthogonal space in orthogonal planes (to be defined in the next section) will be important in what follows.

The $\mathfrak{F}_{\boldsymbol{y} \perp \boldsymbol{x}}$ plane. Consider an arbitrary reference vector $x$ (of maximal rank for simplicity) and a perpendicular vector $y \in \mathfrak{F}_{x}^{\perp}$. We define the space, $\mathfrak{F}_{y \perp x}:=\operatorname{span}\left\{y, \Upsilon_{x}(y)\right\}$. That is

$$
\mathfrak{F}_{y \perp x}:=\left\{a y+b \Upsilon_{x}(y) \mid y \in \mathfrak{F}_{x}^{\perp} \quad a, b \in \mathbb{R}\right\}
$$


We will show that the "planes" $\mathfrak{F}_{x}$ and $\mathfrak{F}_{y \perp x}$ are $\{$,$\} -orthogonal:$

$$
\mathfrak{F}_{y \perp x} \subseteq \mathfrak{F}_{x}^{\perp}
$$

For any $y \in \mathfrak{T}_{x}^{\perp}$, we can show that also $\Upsilon_{x}(y) \in \mathfrak{T}_{x}^{\perp}$. We have indeed (using Equation (11c) in $[14]$ in the first line and axiom 3 in the second line)

$$
\begin{aligned}
& \left\{x^{\prime}, \Upsilon_{x}(y)\right\}=3\{T(x, x, x), T(x, x, y)\}=-\left\{y, x^{\prime}\right\} \Delta(x)=0, \\
& \left\{x, \Upsilon_{x}(y)\right\}=\{x, 3 T(x, x, y)\}=3\{y, T(x, x, x)\}=3\left\{y, x^{\prime}\right\}=0 .
\end{aligned}
$$

which implies $\Upsilon_{x}(y) \in \mathfrak{F}_{x}^{\perp}$. Obviously, the equality $\mathfrak{F}_{y \perp x}=\mathfrak{F}_{x}^{\perp}$ is only possible for $\operatorname{dim}(\mathfrak{F})=$ 4 , as $\operatorname{dim}\left(\mathfrak{F}_{x}^{\perp}\right)=\operatorname{dim}(\mathfrak{F})-2$.

We show next that, in the same case, successive powers of $\Upsilon_{x}$ acting on $y$ belong to the orthogonal plane. In fact, $\Upsilon_{x}^{n}(y)$ is proportional to $y$ or $\Upsilon_{x}(y)$. We have for example (as for any $y \in \mathfrak{F}_{x}^{\perp}$ )

$$
\begin{aligned}
\Upsilon_{x}^{2}(y) \equiv \Upsilon_{x}\left(\Upsilon_{x}(y)\right) & =9 T(x, x, T(x, x, y)) \\
& =-2 \Delta(x, x, x, y) x-\Delta(x) y-\{y, x\} x^{\prime}=-\left\{x^{\prime}, y\right\}-\Delta(x) y \\
& =-\Delta(x) y
\end{aligned}
$$

In the second line we have used the Lemma 1(11e) in [14]. In general for any $n$, we have, by using induction (for any $y \in \mathfrak{F}_{x}^{\perp}$ ), for $n \geq 1$,

$$
\begin{aligned}
\Upsilon_{x}^{2 n}(y) & =(-1)^{n} \Delta(x)^{n} y \\
\Upsilon_{x}^{2 n+1}(y) & =(-1)^{n} \Delta(x)^{n} \Upsilon_{x}(y)
\end{aligned}
$$

Let us remark that $\mathfrak{F}_{y \perp x}$ is not closed in general under $\mathfrak{T}$-transformations, it is not a sub-FTS with the operations inherited from the parent FTS. The plane $\mathfrak{F}_{y \perp x}$ is however closed under the $\Upsilon_{x}$ map. For any element belonging to it $\left(u \in \mathfrak{F}_{y \perp x}, u=a y+b \Upsilon_{x}(y)\right)$,

$$
\begin{aligned}
\Upsilon_{x}(u) & =3 T\left(x, x, a y+b \Upsilon_{x}(y)\right)+\left\{x, a y+b \Upsilon_{x}(y)\right\} x \\
& =3 a T(x, x, y)+3 b T\left(x, x, \Upsilon_{x}(y)\right) \\
& =-b \Delta(x) y+a \Upsilon_{x}(y)
\end{aligned}
$$

where in the second line we have used the equality expressed by eq. (4.16) (see also [14]). According to this $\Upsilon_{x}(u) \in \mathfrak{F}_{x}^{\perp}$.

\subsection{Behaviour of $\Delta$ on the $\mathfrak{F}_{y \perp x}$ plane}

We are interested in the behaviour of $\Delta$ on the $\mathfrak{F}_{y \perp x}$ plane. For any $u \in \mathfrak{F}_{y \perp x}, u=$ $a y+b \Upsilon_{x}(y)$, we have, by combining eq. (B.10) with eq. (B.11)

$$
\begin{aligned}
\Delta(u) & =\left(a^{2}+b^{2} \Delta(x)\right)^{2} \Delta(y), \\
\Delta\left(\Upsilon_{x}(y)\right) & =\Delta(x)^{2} \Delta(y) .
\end{aligned}
$$


Or, in normalized terms

$$
\Delta\left(\tilde{\Upsilon}_{x}(y)\right) \equiv \Delta\left(\frac{\Upsilon_{x}(y)}{\sqrt{|\Delta(x)|}}\right)=\Delta(y) .
$$

The $\tilde{\Upsilon}_{x}$ map thus preserves both the bilinear and quartic invariants in each of the $\mathfrak{F}_{y \perp x}$ planes. Applying twice eq. (4.25) we arrive to

$$
\Delta\left(\tilde{\Upsilon}_{x}^{2}(y)\right)=\Delta(y)
$$

and in general

$$
\Delta\left(\tilde{\Upsilon}_{x}^{n}(y)\right)=\Delta(y)
$$

Combining eqs. (4.23), (4.24) with (3.9) we arrive to an expression

$$
\Delta\left(a y+b \Upsilon_{x}(y)\right) \Delta(x)=\Delta\left(a+b x^{\prime}\right) \Delta(y) .
$$

which relates the behaviour of the quartic invariant $\Delta$ on the $\mathfrak{T}_{x}$ and $\mathfrak{F}_{y \perp x}$ planes.

The behaviour of $\Delta$ on the $\mathfrak{F}_{x}$ or $\mathfrak{F}_{y \perp x}$ planes is similar but with some important differences. In the $\mathfrak{F}_{y \perp x}$ case it depends on the signs of both $\Delta(x)$ and $\Delta(y)$. The overall sign of all the elements of the $\mathfrak{F}_{y \perp x}$ plane is the same as $\Delta(y)$ excluding the null elements such that

$$
a^{2}+b^{2} \Delta(x)=0
$$

For example, any element of the form

$$
z_{ \pm} \propto \sqrt{|\Delta(x)|} y \pm \Upsilon_{x}(y)
$$

is null, $\Delta\left(z_{ \pm}\right)=0$ (for $\Delta(x)<0, y \in \mathfrak{F}_{x}^{\perp}$ ). We observe that the null elements of $\mathfrak{F}_{x}$ and any $\mathfrak{F}_{y \perp x}$ are aligned, they are given by the same eq. (4.29) which it is independent of $y$.

\section{Freudenthal rotations: the $\Upsilon$ map and its exponential}

The closure of $\mathfrak{F}_{x}$ under F-duality implies the existence of a one-parameter family of Uduality transformations stabilising $\mathfrak{F}_{x}$ as it will be shown in this section.

Recall, for any fixed $x, \Upsilon_{x}$ is in $\mathfrak{a u t}(\mathfrak{F})$. In particular, the normalised version, $\bar{\Upsilon}_{x}$, given in eq. (2.7), acting on $x$ itself maps $x$ into its F-dual,

$$
\bar{\Upsilon}_{x}(x)=\epsilon \tilde{x} .
$$

Note, we also have the relation $(\epsilon=\operatorname{sgn} \Delta(x))$

$$
\begin{aligned}
\Upsilon_{\tilde{x}}(x) & =3 T(\tilde{x}, \tilde{x}, x)+\{\tilde{x}, x\} \tilde{x} \\
& =\frac{1}{|\Delta(x)|}\left(3 T\left(x^{\prime}, x^{\prime}, x\right)+\left\{x^{\prime}, x\right\} x^{\prime}\right), \\
& =\frac{1}{|\Delta(x)|}\left(\Delta(x) x^{\prime}+2 \Delta(x) x^{\prime}\right)=3 \epsilon x^{\prime},
\end{aligned}
$$


then

$$
\bar{\Upsilon}_{\tilde{x}}(x)=\frac{\epsilon}{\sqrt{\Delta(\tilde{x}) \mid}} x^{\prime}=\tilde{x} .
$$

It is obvious that $\Upsilon_{x}$ (as well as $\Upsilon_{a x+b \tilde{x}}$ ) is a $\mathfrak{F}_{x} \rightarrow \mathfrak{F}_{x}$ map. Furthermore, the set of maps $\left\{\Upsilon_{a x+b \tilde{x}}\right\}_{a, b \in \mathbb{R}}$ for a fixed $x$ forms an two-parametric automorphism subalgebra.

We are interested here in the action of the $\Upsilon_{x}$ map and the computation of its exponential. For this purpose, it is convenient to distinguish the action of any $\Upsilon_{x}$ on its particular associated $\mathfrak{F}_{x}$ plane and on the respective orthogonal complement $\mathfrak{F}_{x}^{\perp} .{ }^{9}$

\subsection{The exponential map on the $\mathfrak{F}_{x}$-plane}

The action of the exponential of the (normalized) map $\bar{\Upsilon}_{x}$ reads as follows. For any rank-4, fixed, $x \in \mathfrak{F},(\theta \in \mathbb{F}, \epsilon(x)=\operatorname{sgn} \Delta(x))$

$$
\begin{aligned}
& \exp \left(\theta \bar{\Upsilon}_{x}\right)(x)=\cos (\sqrt{\varepsilon} \theta) x+\sqrt{\varepsilon} \sin (\sqrt{\varepsilon} \theta) \tilde{x}, \\
& \exp \left(\theta \bar{\Upsilon}_{x}\right)(\tilde{x})=-\varepsilon \sqrt{\varepsilon} \sin (\sqrt{\varepsilon} \theta) x+\cos (\sqrt{\varepsilon} \theta) \tilde{x}, \\
& \exp \left(\theta \bar{\Upsilon}_{\tilde{x}}\right)(x)=\cos (\sqrt{\varepsilon} \theta) x+\varepsilon \sqrt{\varepsilon} \sin (\sqrt{\varepsilon} \theta) \tilde{x}, \\
& \exp \left(\theta \bar{\Upsilon}_{\tilde{x}}\right)(\tilde{x})=-\sqrt{\varepsilon} \sin (\sqrt{\varepsilon} \theta) x+\cos (\sqrt{\varepsilon} \theta) \tilde{x},
\end{aligned}
$$

where $x \in \mathfrak{F}$ and exp is defined by the usual infinite series.

The proof of eqs. (5.6)-(5.9) is based in the following properties:

$$
\begin{aligned}
\Upsilon_{x}^{2 n}(x) & =(-1)^{n}(3)^{2 n} \Delta(x)^{n} x, \\
\Upsilon_{x}^{2 n+1}(x) & =(-1)^{n}(3)^{2 n+1} \Delta(x)^{n} x^{\prime}, \\
\Upsilon_{\tilde{x}}^{2 n}(x) & =\Upsilon_{x}^{2 n}(x), \\
\Upsilon_{\tilde{x}}^{2 n+1}(x) & =\varepsilon \Upsilon_{x}^{2 n+1}(x),
\end{aligned}
$$

which are obtained by induction starting on with $\Upsilon_{x}(x)=3 x^{\prime}, \Upsilon_{x}^{2}(x)=9 T\left(x, x, x^{\prime}\right)=$ $-3 \Delta(x) x$. By linearity $\exp \theta \Upsilon_{x}$ can be extended to the full $\mathfrak{F}_{x}$ plane.

Explicitly, for $\Delta_{x}=\Delta_{\tilde{x}}>0 \Leftrightarrow \varepsilon=1$, it holds that

$$
\begin{aligned}
& \exp \left(\theta \bar{\Upsilon}_{x}\right)(x)=\cos (\theta) x+\sin (\theta) \tilde{x}, \\
& \exp \left(\theta \bar{\Upsilon}_{x}\right)(\tilde{x})=-\sin (\theta) x+\cos (\theta) \tilde{x}, \\
& \exp \left(\theta \bar{\Upsilon}_{\tilde{x}}\right)(x)=\cos (\theta) x+\sin (\theta) \tilde{x}=\exp \left(\theta \bar{\Upsilon}_{x}\right)(x), \\
& \exp \left(\theta \bar{\Upsilon}_{\tilde{x}}\right)(\tilde{x})=-\sin (\theta) x+\cos (\theta) \tilde{x}=\exp \left(\theta \bar{\Upsilon}_{x}\right)(\tilde{x}),
\end{aligned}
$$

whereas for $\Delta_{x}=\Delta_{\tilde{x}}<0 \Leftrightarrow \varepsilon=-1$, it holds that

$$
\begin{aligned}
& \exp \left(\theta \bar{\Upsilon}_{x}\right)(x)=\cosh (\theta) x-\sinh (\theta) \tilde{x}, \\
& \exp \left(\theta \bar{\Upsilon}_{x}\right)(\tilde{x})=-\sinh (\theta) x+\cosh (\theta) \tilde{x}, \\
& \exp \left(\theta \bar{\Upsilon}_{\tilde{x}}\right)(x)=\cosh (\theta) x+\sinh (\theta) \tilde{x}, \\
& \exp \left(\theta \bar{\Upsilon}_{\tilde{x}}\right)(\tilde{x})=\sinh (\theta) x+\cosh (\theta) \tilde{x} .
\end{aligned}
$$

\footnotetext{
${ }^{9}$ For convenience, we work indistinctly on the $\mathfrak{F}_{x}, \mathfrak{F}_{x}^{\perp}$ or on $\mathfrak{T}_{x}, \mathfrak{T}_{x}^{\perp}$. They are equivalent as long $\Delta(x) \neq 0$.
} 
Hence, the set of transformations $\exp \theta \Upsilon_{x}$ form an automorphism subgroup $\operatorname{Aut}\left(\mathfrak{F}_{x}\right) \subseteq$ $\operatorname{Aut}(\mathfrak{F})$ preserving the $\mathfrak{F}_{x}$ plane.

To summarise, as a consequence of eqs. (5.6) and (5.7), for any rank-4 $x \in \mathfrak{F}$, there exists a monoparametric subgroup $\sigma_{x}(\theta) \in \operatorname{Aut}\left(\mathfrak{F}_{x}\right)$ which is made of "rotations" in $\mathfrak{F}_{x}$ and whose generator is $\Upsilon_{x}$ :

$$
\sigma_{x}(\theta) \equiv e^{\theta \bar{\Upsilon}_{x}}
$$

Let us study the details of the automorphism subgroup $\sigma_{x}(\theta)$ depending on the sign of $\Delta(x)$. For $\Delta(x)>0,(\epsilon=1)$ the subgroup $\sigma_{x}(\theta)$ is $\mathrm{SO}(2)$. The Freudenthal rotation with $\theta=\pi / 2$ is the U-duality transformation relating $x$ to its F-dual. For $\mathcal{N}=8$ black holes with $G_{4}=E_{7(7)}$ the existence of a U-duality connecting $x$ and $\tilde{x}$ was guaranteed since all $x$ with the same $\Delta(x)>0$ belong to the same $E_{7(7)}$ orbit. For $\mathcal{N}<8$ not all $x$ with the same $\Delta(x)>0$ necessarily lie in the same U-duality orbit; the orbits are split by further U-duality invariant conditions. See [45, 59] and the references therein. Nonetheless, for $\epsilon=1$ the Freudenthal rotation given by eq. (5.6) with $\theta=\pi / 2$ implies that $x$ and $\tilde{x}$ are in the same U-duality orbits for all FTS.

On the other hand, for $\Delta(x)<0,(\epsilon=-1)$ the subgroup $\sigma_{x}(\theta)$ is $S O_{0}(1,1)$ which has three different kinds of orbits: the origin (a group fixed point), the four rays $\{( \pm t, \pm t), t>$ $0\}$, and the hyperbolae $a^{2}-b^{2}= \pm r^{2}$. The Freudenthal rotation cannot relate $x$ to its F-dual (by inspection of eq. (5.7), the orbits of the exponential of the $\Upsilon$ are hyperbolic). Therefore $x, \tilde{x}$ lie in different branches. However, for any FTS, all $x$ with the same $\Delta(x)<0$ lie in the same U-duality orbit $[45,59]$. Hence, there exists a U-duality transformation, which is determined by $x$, connecting $x$ and $\tilde{x}$ for $\Delta(x)<0$. But, this U-duality transformation is not represented by any member of $\sigma_{x}(\theta)$. In fact, as we shall see there is a one-parameter family of U-dualities which connects $x$ and $\tilde{x}$ but does not preserve the $\mathfrak{F}_{x}$-plane. We will return to this question in the next sections.

In summary, putting together the previous comments, we arrive to the conclusion that

a) For all supergravities with $E_{7}$-type duality group of any $\mathcal{N}$, large $\mathrm{BH}$ have charges $x$ and $\tilde{x}$ in the same U-duality orbit, irrespective of the sign of $\Delta(x)$.

b) For $\Delta(x)>0$ the orbit of $\sigma_{x}(\theta)$, which relates the F-dual BHs, is contained in the $\mathfrak{F}_{x}$-plane. For $\Delta(x)<0$, the orbit of of the one-parameter subgroup, introduced later, connecting $x$ and $\tilde{x}$ does not preserve the $\mathfrak{F}_{x}$-plane. It would perhaps be "natural" to conjecture that this orbit only intersects the $\mathfrak{F}_{x}$-plane only at $x$ and $\tilde{x}$. We will come back to this point later on.

Note, a similar treatment can be performed for the case of small BHs, $\Delta(x)=0$. In this case the group generated by $\Upsilon_{x}$ has orbits corresponding to null rays. 


\section{Pure $\mathcal{N}=2, D=4$ supergravity and degenerate FTS}

The simplest example of a FTS (which is, being two-dimensional, a Freudenthal plane with $\Delta(x)>0$ ) in supergravity is provided by the one associated to "pure" $\mathcal{N}=2, D=4$ supergravity, whose purely bosonic sector is the simplest (scalarless) instance of MaxwellEinstein gravity. In such a theory, the asymptotically flat, spherically symmetric, dyonic extremal Reissner-Nordström (RN) black hole (BH) solution has Bekenstein-Hawking entropy

$$
\frac{S_{R N}}{\pi}=\frac{1}{2}\left(p^{2}+q^{2}\right)
$$

where $p$ and $q$ are the magnetic resp. electric fluxes associated to the unique Abelian vector field (which, in the $\mathcal{N}=2$ supersymmetric interpretation, is the so-called graviphoton).

In this case, the associated FTS $\mathfrak{F}_{\mathcal{N}=2}$ "pure" has $\operatorname{dim}=2$ (i.e., it has $N=1$, within the previous treatment); it is immediate to realize that this cannot be a reduced FTS, because $^{10}$ defining

$$
x=(p, q)^{T}
$$

then the associated quartic invariant $\Delta(x)$ is defined by

$$
\Delta(x)=\frac{1}{4}\left(p^{2}+q^{2}\right)^{2}=\frac{S_{R N}^{2}}{\pi^{2}} \geqslant 0
$$

for any choice of $p$ and $q$.

This system can be considered a BPS $(\Delta(x)>0)$ prototype. Let us start by doing some, simple, explicit computations. For this purpose let us choose (without any loss of generality) a vector given by

$$
x=(p, 0)^{T},
$$

which corresponds to a purely magnetic extremal RN BH. For this configuration,

$$
\begin{aligned}
\Delta(x) & =\frac{1}{4} p^{4}, \\
\frac{S_{R N}(x)}{\pi} & =\frac{1}{2} p^{2} .
\end{aligned}
$$

Introducing a basis ${ }^{11}\left\{e_{M}\right\}_{\mathcal{M}=1}^{\operatorname{dim} \mathfrak{F}}$, the Freudenthal dual $\widetilde{x}$ of $x$ can be computed $[17,19]$ by using

$$
\widetilde{x}^{M}=\Omega^{M N} \frac{1}{\pi} \frac{\partial S(x)}{\partial x^{N}}=\Omega^{M N} \frac{\partial \sqrt{|\Delta(x)|}}{\partial x^{N}}=\frac{\epsilon}{2 \sqrt{\epsilon \Delta(x)}} \Omega^{M N} \frac{\partial \Delta(x)}{\partial x^{N}},
$$

where we recall that $\epsilon \equiv \operatorname{sgn} \Delta(x)$. Note, we have introduce here the $\operatorname{dim} \mathfrak{F} \times \operatorname{dim} \mathfrak{F}=$ $2 N \times 2 N$ symplectic matrix $\Omega$, defined by

$$
\{x, y\} \equiv x^{T} \Omega y
$$

\footnotetext{
${ }^{10}$ The case $\Delta=0$ corresponds to the uncharged limit $p=0=q$.

${ }^{11}$ For $\operatorname{dim} \mathfrak{F}=2 N$, Latin capital indices are symplectic, and take values over $0,1, \ldots, N-1$ contravariant and $0,1, \ldots, N-1$ covariant indices.
} 
For a generic FTS, we can choose a basis such that $\Omega$ is realized as follows:

$$
\Omega=\left(\Omega^{M N}\right)=\left(\begin{array}{cc}
\mathbf{0} & -\mathbb{1} \\
\mathbb{1} & \mathbf{0}
\end{array}\right),
$$

where $\mathbf{0}$ and $\mathbb{1}$ denote the $N \times N$ zero and identity matrices, respectively.

In the present case

$$
x^{T} \Omega y=q p^{\prime}-p q^{\prime}, \quad x=(p, q)^{T}, y=\left(p^{\prime}, q^{\prime}\right)^{T}
$$

and from eq. (6.3) we find

$$
\widetilde{x} \equiv(\tilde{p}, \tilde{q})^{T}=(0, p)^{T} .
$$

A purely electric extremal RN BH is nothing else as the Freudenthal dual of purely magnetic extremal RN BH. The whole FTS $\mathfrak{F}_{\mathcal{N}=2}$ "pure" coincides with the Freudenthal plane $\mathfrak{F}_{x}$ associated to $x$ :

$$
\mathfrak{F}_{\mathcal{N}=2} \text { "pure" }=\mathfrak{F}_{x}=\{a x+b \widetilde{x}, a, b \in \mathbb{R}\}
$$

and transverse space is obviously empty $\mathfrak{F}_{x}^{\perp}=\varnothing$. A general Freudenthal transformation (GFT) depending on the real parameters $a, b$ is given by

$$
x \rightarrow x_{F}=a x+b \widetilde{x}
$$

or, in this case

$$
x_{F}=\left(q_{F}, p_{F}\right)^{T}=(a p, b p)^{T},
$$

with

$$
\begin{aligned}
\Delta\left(x_{F}\right) \equiv \Delta(a x+b \widetilde{x}) & =\frac{1}{4}\left(a^{2} p^{2}+b^{2} q^{2}\right)^{2} \\
& =\frac{1}{4}\left(a^{2}+b^{2}\right) p^{4} \geqslant 0 .
\end{aligned}
$$

The corresponding extremal RN BH is supersymmetric and $\frac{1}{2}$-BPS (in absence of scalar fields, supersymmetry implies extremality). For $a^{2}+b^{2}=1$, the general Freudenthal transformation leaves invariant the entropy of the black hole. In this context a GFT is nothing else as an instance of EM duality. Automorphism algebra and group element as $\Upsilon$ and $\exp (\theta \Upsilon)$ can be explicitly and easily computed. $\Upsilon_{x}(a x+b \tilde{x}) \propto \widehat{a x+b \tilde{x}}$.

$\mathfrak{F}_{\mathcal{N}=2}$ "pure" provides the simplest case of degenerate FTS, in which $2 \Delta$ is the square of a quadratic polynomial $I_{2}$ :

$$
\Delta=I_{2}^{2},
$$

and thus it is always positive. In fact, "pure" $\mathcal{N}=2, D=4$ supergravity is the $n_{V}=0$ limit of the sequence of $\mathcal{N}=2, D=4$ supergravity "minimally coupled" to $n_{V}$ vector multiplets $^{12}$ [61] (see also [62, 63]), in which the related FTS is degenerate $\forall n_{V} \in \mathbb{N} \cup\{0\}$; the corresponding scalar manifold is $\overline{\mathbb{C P}}^{n_{V}}$.

\footnotetext{
${ }^{12}$ Actually, such a sequence is the unique, at least among theories with homogeneous scalar manifolds, to admit the "pure" theory as the limit of $n_{V}=0$ vector multiplets.
} 
In the formalism discussed in section 3 , in $\mathfrak{F}_{\mathcal{N}=2}$ "pure" $=\mathfrak{F}_{x}$ it holds that $(I, J=1,2)$

$$
I_{2}=\frac{1}{2}\|\mathbf{x}\|_{\delta}^{2}=\frac{1}{2} \delta_{I J} x^{I} x^{J},
$$

with $x^{1}=x, x^{2}=\widetilde{x}$. The Euclidean nature of the metric structure defined on $\mathfrak{F}_{\mathcal{N}=2}$ "pure" $=$ $\mathfrak{F}_{x}$ corresponds to a spherical foliation of $\mathfrak{F}_{x}$ for $\Delta>0$.

Degenerate FTS's never satisfy the reducibility condition [14], namely they are globally non-reduced; they have been treated e.g. in [64], and their application in supergravity has been discussed in [27] (see also and [63]). Other (infinite) examples of degenerate FTS's are provided by the ones related to the $n$-parameterised sequence of $\mathcal{N}=3, D=4$ supergravity coupled to $n$ matter (vector) multiplets $[27,62,65]$. On the other hand, $\mathcal{N}=4[66]$ and $\mathcal{N}=5,{ }^{13} D=4$ "pure" supergravities have FTS's which do not satisfy the degeneracy condition eq. (6.17) in all symplectic frames, but rather eq. (6.17) is satisfied at least in the so-called "scalar-dressed" symplectic frame [62].

This FTS cannot be associated to any Jordan Algebra. Consistently, "pure" $\mathcal{N}=2$, $D=4$ supergravity does not admit an uplift to $D=5$, or conversely it cannot be obtained by dimensionally reducing any $D=5$ theory down to $D=4$. In general, degenerate FTS's are not built starting from rank-3 Jordan algebras, and therefore the corresponding Maxwell-Einstein (super)gravity models do not admit an uplift to $D=5$; rather, degenerate FTS's are based on Hermitian (Jordan) triple systems (cfr. e.g. [43, 64], and refs. therein).

As discussed in section 10 of [27], at least for the degenerate FTS's relevant to $D=4$ supergravities with symmetric scalar manifold (i.e., $\mathcal{N}=2$ "minimally coupled" and $\mathcal{N}=$ 3 theories ${ }^{14}$ ), Freudenthal duality is nothing but an anti-involutive U-duality mapping. This can be realized immediately in the aforementioned case of $\mathcal{N}=2, D=4$ "pure" supergravity; let us consider $(a=b=1)$

$$
\mathfrak{F}_{\mathcal{N}=2} \text { "pure" } \ni y=(p, q)^{T} \Rightarrow \Delta(x+\widetilde{x})=\frac{1}{2}\left(p^{2}+q^{2}\right)^{2} .
$$

The Freudenthal dual $\widetilde{y}$ of $y$ can be computed (by recalling eq. (6.7) an using eq. (6.3)) to read

$$
\widetilde{y}=(-q, p)^{T}=\Omega_{0} y,
$$

where $\Omega_{0}$ is nothing but the canonical symplectic $2 \times 2$ metric $\Omega_{2 \times 2}$ :

$$
\Omega_{0} \equiv\left(\begin{array}{cc}
0 & -1 \\
1 & 0
\end{array}\right) \equiv \Omega_{2 \times 2} .
$$

Thus, Freudenthal duality in $\mathfrak{F}_{\mathcal{N}=2}$ "pure" is given by the application of the symplectic metric $\Omega \equiv \Omega_{0}$, and it is thus an anti-involutive $U$-duality transformation. The relation

\footnotetext{
${ }^{13}$ A particularly interesting case is provided by $\mathcal{N}=5, D=4$ supergravity [67], which is seemingly related to a non-reduced FTS which is non-degenerate, but also to a triple system denoted by $M_{2,1}(\mathbb{O}) \sim$ $M_{1,2}(\mathbb{O})[42,68,69]$ which deserves a particular study.

${ }^{14}$ These cases pertain to simple, degenerate FTS's [27]. No examples of semi-simple or non-semi-simple degenerate FTS's relevant to (super)gravity $(D=4)$ models are known to us.
} 
eq. (6.20) defines a $\mathbb{Z}_{4}$ symmetry in the 2-dim. FTS $\mathfrak{F}_{\mathcal{N}=2}$ "pure" $=\mathfrak{F}_{x}$, spanned by $x$ eq. (6.6) and its Freudenthal dual $\widetilde{x}$ eq. (6.11): in fact, the iteration of Freudenthal duality yields

$$
(p, q)^{T} \stackrel{\sim}{\rightarrow}(-q, p)^{T} \stackrel{\sim}{\rightarrow}-(p, q)^{T} \stackrel{\sim}{\rightarrow}(q,-p)^{T} \stackrel{\sim}{\rightarrow}(p, q)^{T} .
$$

This provides the realisation of the $\mathbb{Z}_{4}$ in the FTS $\mathfrak{F}_{\mathcal{N}=2}$ "pure" $=\mathfrak{F}_{x}$, as a consequence of the anti-involutivity of Freudenthal duality itself. The same symmetry will be also explicitly observed, for example, for the Freudenthal plane defined by the $D 0-D 6$ brane charge configuration in reduced FTS's, to be studied in latter sections.

\section{The axion-dilaton $\mathcal{N}=2, D=4$ supergravity}

Let us consider now $\mathcal{N}=2, D=4$ supergravity "minimally coupled" to one vector multiplet, in the so-called axion-dilaton (denoted by the subscript "ad") symplectic frame. Ultimately, this is nothing but the $n_{V}=1$ element of the sequence of $\overline{\mathbb{C P}}^{n_{V}}$ "minimally coupled" models [61], but in a particular symplectic frame, which can be obtained as a consistent truncation of "pure" $\mathcal{N}=4$ supergravity, in which only two of the six graviphoton survive (in this frame, the holomorphic prepotential reads $F(X)=-i X^{0} X^{1}$; cfr. e.g. the discussion in [70], and refs. therein).

The purely bosonic sector of such an $\mathcal{N}=2$ theory may be regarded as the simplest instance of Maxwell-Einstein gravity coupled to one complex scalar field. In the axiondilaton symplectic frame, in the particular charge configuration obtained by setting to zero two charges out of four and thus having only two non-vanishing charges, ${ }^{15}$ namely one magnetic and one electric charge $p$ resp. $q$, the asymptotically flat, spherically symmetric, dyonic extremal BH solution has Bekenstein-Hawking entropy

$$
\frac{S_{a d}}{\pi}=|p q|
$$

and it is non-supersymmetric ${ }^{16}$ (non-BPS). The expression eq. (7.1) is very reminiscent of the Bekenstein-Hawking entropy of a $\mathrm{BH}$ in a reduced FTS in the $D 0-D 6$ charge configuration (to be treated later on, we refer to eqs. (8.3)-(8.4), $I_{4} \equiv \Delta(x)$ ):

$$
\frac{S_{B H}}{\pi}=\sqrt{|\Delta|}=\left|p^{0} q_{0}\right|
$$

However, the $\mathcal{N}=2$ axion-dilaton supergravity model, as the "pure" $\mathcal{N}=4, D=4$ supergravity from which it derives, cannot be uplifted to $D=5$ (as instead all models related to reduced FTS's can), consistently with its "minimally coupled" nature: in fact, the charges $P$ and $Q$ do not have the interpretation of the magnetic resp. electric charge of the KK vector in the $D=5 \rightarrow 4$ dimensional reduction.

\footnotetext{
${ }^{15}$ In this case, the effective FTS $\mathfrak{F}_{\mathcal{N}=2}$ ad given by the truncation has dimension 2 .

${ }^{16}$ Indeed, in presence of scalar fields (in this context stabilized at the event horizon of the BH by virtue of the attractor mechanism), extremality does not imply BPS nature, and extremal non-BPS solutions may exist.
} 
This truncated system can be described by a two dimensional FTS characterized by a quartic form $\left(x \equiv(p, q)^{T}\right)$

$$
\Delta(x)=-\frac{1}{2}(p q)^{2} .
$$

One can recast this expression by defining

$$
p \equiv \frac{1}{\sqrt{2}}(P+Q), \quad q \equiv \frac{1}{\sqrt{2}}(P-Q),
$$

in the following form

$$
\frac{S_{a d}}{\pi}=\frac{1}{2}\left|P^{2}-Q^{2}\right|
$$

Let us start by choosing, without any loss of generality, a charge configuration given by

$$
x=(P, 0)^{T} .
$$

The corresponding entropy is given by

$$
\frac{S_{a d}(x)}{\pi}=\frac{1}{2} P^{2} .
$$

By virtue of eq. (6.7), one can compute the Freudenthal dual $\widetilde{x}$ of $x$ to $\operatorname{read}(\epsilon \equiv$ $\operatorname{sgn}\left(P^{2}-Q^{2}\right)$

$$
\widetilde{x}=(\tilde{P}, \tilde{Q})=(0, \epsilon P)^{T}
$$

Thus, one can define a GFT transformations and the 2-dim. Freudenthal plane $\mathfrak{F}_{x}$ associated to $x$ inside the whole 4 -dim. FTS $\mathfrak{F}_{\mathcal{N}=2 \text { ad }}$ :

$$
\mathfrak{F}_{\mathcal{N}=2 \text { ad }} \supset \mathfrak{F}_{x}=\left\{x_{F} \equiv a x+b \widetilde{x}, a, b \in \mathbb{R}\right\},
$$

with

$$
\frac{S_{a d}\left(x_{F}\right)}{\pi}=\frac{1}{2}\left|a^{2} P^{2}-b^{2} Q^{2}\right| .
$$

The corresponding extremal $\mathrm{BH}$ is non-supersymmetric (non-BPS). In particular for the eq. (7.6) configuration

$$
\frac{S_{a d}\left(x_{F}\right)}{\pi}=\frac{1}{2}\left|a^{2}-Q^{2}\right| \frac{S_{a d}(x)}{\pi} .
$$

The entropy is invariant for $a^{2}-b^{2}= \pm 1$.

Within the formalism discussed in section 3 , in $\mathfrak{F}_{x} \subset \mathfrak{F}_{\mathcal{N}=2 \text { ad }}$, it holds that

$$
I_{2}=\frac{1}{2}\|\mathbf{x}\|_{\eta}^{2}=\frac{1}{2} \eta_{I J} x^{I} x^{J}
$$

with $x^{1}=x, x^{2}=\widetilde{x}$, and $\eta_{I J}=\operatorname{diag}(1,-1)$. The Kleinian nature of the metric structure defined on $\mathfrak{F}_{x} \subset \mathfrak{F}_{\mathcal{N}=2 \text { ad }}$ corresponds to an hyperbolic (i.e., hyperboloid-like) foliation of $\mathfrak{F}_{x}$ for $\Delta<0$. Therefore, notwithstanding the fact that $\mathcal{N}=2, D=4$ axion-dilaton supergravity is nothing but the $\overline{\mathrm{CP}}^{1}$ "minimally coupled" model in a particular (nonFubini-Study) symplectic frame and thus with eq. (6.17) holding true, in the peculiar 
$(P, Q)$ charge configuration eq. (7.4), the corresponding $\mathfrak{F}_{x} \subset \mathfrak{F}_{\mathcal{N}=2 \text { ad }}$ can be considered as a "degenerate" limit of the $\Delta<0$ prototype of Freudenthal plane for reduced FTS's.

It is instructive to consider the explicit action of the Freudenthal duality in the Freudenthal plane $\mathfrak{F}_{x} \subset \mathfrak{F}_{\mathcal{N}=2 \text { ad }}$. Let us start and consider $(a=b=1$; we disregard the coordinates in $\mathfrak{F}_{\mathcal{N}=2 \text { ad }}$ pertaining to $\mathfrak{F}_{x}^{\perp}=\mathfrak{F}_{\mathcal{N}=2 \text { ad }} / \mathfrak{F}_{x}$ )

$$
\mathfrak{F}_{\mathcal{N}=2 \text { ad }} \supset \mathfrak{F}_{x} \ni y=(P, Q)^{T} \Rightarrow \frac{S_{a d}(y)}{\pi}=\frac{1}{2}\left|P^{2}-Q^{2}\right| .
$$

The Freudenthal dual $\widetilde{y}$ of $y$ can be computed (by recalling eq. (6.7)) to $\operatorname{read}^{17}$

$$
\widetilde{y}=\epsilon(Q, P)^{T}=\epsilon \hat{\mathbf{O}} y,
$$

with

$$
\hat{\mathbf{O}}:=\left(\begin{array}{ll}
0 & 1 \\
1 & 0
\end{array}\right) \text {. }
$$

Note that $\mathbf{O}$ eq. (7.15) is involutive:

$$
\hat{\mathbf{O}}^{2}=I d
$$

but since the Freudenthal duality on $\mathfrak{F}_{x}$ exchanges $P$ and $Q$ and thus flips $\epsilon(=$ $\left.\operatorname{sgn}\left(P^{2}-Q^{2}\right)\right)$, it follows that the correct iteration of the Freudenthal duality on $\mathfrak{F}_{x} \subset$ $\mathfrak{F}_{\mathcal{N}=2 \text { ad }}$ is provided by the application of $\epsilon \hat{\mathbf{O}}$ and then necessarily of $-\epsilon \hat{\mathbf{O}}$, thus corresponding to $-\hat{\mathbf{O}}^{2}=-I d$ acting on $x$, and thus correctly yielding

$$
\widetilde{\widetilde{x}}=-x \text {. }
$$

As at the end of previous subsection for "pure" $\mathcal{N}=2, D=4$ supergravity, in this case due to the relations eqs. (7.14)-(7.15), we can define a $\mathbb{Z}_{4}$ symmetry in the 2$\operatorname{dim}$. Freudenthal plane $\mathfrak{F}_{x} \subset \mathfrak{F}_{\mathcal{N}=2 \text { ad }}$, spanned by $x$ eq. (7.7) and its Freudenthal dual $\widetilde{x}$ eq. (7.8): e.g., starting from $\epsilon=1$, the iteration of Freudenthal duality yields

$$
(P, Q)^{T} \stackrel{\sim}{\rightarrow}(Q, P)^{T} \stackrel{\sim}{\rightarrow}-(P, Q)^{T} \stackrel{\sim}{\rightarrow}-(Q, P)^{T} \stackrel{\sim}{\rightarrow}(P, Q)^{T} .
$$

This provides the realisation of the $\mathbb{Z}_{4}$ in the Freudenthal plane $\mathfrak{F}_{x} \subset \mathfrak{F}_{\mathcal{N}=2 \text { ad }}$, as a consequence of the anti-involutivity of Freudenthal duality itself.

\section{$8 \mathcal{N}=2, D=4$ supergravities from $D=5$ : the reduced $\mathfrak{F}$ case}

We will now proceed to present an analysis of the (non-degenerate) reduced FTS's, of the properties of Freudenthal duality defined in them, and of the corresponding Freudenthal planes.

\footnotetext{
${ }^{17} \mathrm{By}$ virtue of the discussion made at the end of th previous subsection (also cfr. section 10 of [27]), $\mathbf{O}$ eq. (7.15) can be completed to a $4 \times 4$ (consistently anti-involutive; cfr. discussion further below) transformation of the U-duality group $\mathrm{U}(1,1)$.
} 
Unless otherwise noted, we will essentially confine ourselves at least to (non-degenerate) reduced FTS's $\mathfrak{F}=\mathfrak{F}\left(J_{3}\right)$, for which a $4 D / 5 D$ special coordinates' symplectic frame can be defined.

A generic element $x$ of the reduced FTS $\mathfrak{F}$ splits as

$$
x=\left(x^{0}, x^{i}, x_{0}, x_{i}\right)^{T} \equiv\left(p^{0}, p^{i}, q_{0}, q_{i}\right)^{T},
$$

where the second renaming pertains to the identification of $x$ with a dyonic charge configuration in $D=4$ (super)gravity, where $p$ 's and $q$ 's are magnetic and electric charges, respectively; within the standard convention in supergravity, $p^{0}, p^{i}, q_{0}$ and $q_{i}$ will usually be called $D 6, D 4, D 2, D 0$ (brane) charges, respectively.

In the canonical basis the symplectic product of two generic elements $x$ and $y$ in $\mathfrak{F}$ reads

$$
\{x, y\} \equiv x^{T} \Omega y=-x^{0} y_{0}-x^{i} y_{i}+x_{0} y^{0}+x_{i} y^{i}
$$

where $\Omega$ is a symplectic matrix.

At least within (non-degenerate) reduced FTS's, the quartic polynomial invariant $I_{4}=$ $2 \Delta(x)$ of $\operatorname{Aut}\left(\mathfrak{F}\left(J_{3}\right)\right) \approx \operatorname{Conf}\left(J_{3}\right)$ can be written $^{18}$ as follows ${ }^{19}$ (cfr. e.g. [44, 72, 73]; $(i=1, \ldots, N-1, \operatorname{dim} \mathfrak{F}=2 N))$ :

$$
\begin{aligned}
\Delta(x) \equiv I_{4}(x) & =-\left(p^{0} q_{0}+p^{i} q_{i}\right)^{2}+4 q_{0} I_{3}(p)-4 p^{0} I_{3}(q)+4\left\{I_{3}(p), I_{3}(q)\right\} \\
& =-\left(p^{0} q_{0}+p^{i} q_{i}\right)^{2}+\frac{2}{3} q_{0} d_{i j k} p^{i} p^{j} p^{k}-\frac{2}{3} p^{0} d^{i j k} q_{i} q_{j} q_{k}+d_{i j k} d^{i l m} p^{j} p^{k} q_{l} q_{m}
\end{aligned}
$$

where

$$
\begin{aligned}
I_{3}(p) & \equiv \frac{1}{3 !} d_{i j k} p^{i} p^{j} p^{k}, \\
I_{3}(q) & \equiv \frac{1}{3 !} d^{i j k} q_{i} q_{j} q_{k}, \\
\left\{I_{3}(p), I_{3}(q)\right\} & \equiv \frac{\partial I_{3}(p)}{\partial p^{i}} \frac{\partial I_{3}(q)}{\partial q_{i}} .
\end{aligned}
$$

The symmetric quantities $d^{i j k}, d_{i j k}$ follow the so-called adjoint identity of the Jordan algebra $J_{3}$ underlying the reduced FTS $\mathfrak{F}$ (cfr. e.g. [46, 73, 74] and refs. therein), which reads

$$
d_{(i j \mid k} d_{l \mid m n)} d^{k l p}=\frac{4}{3} \delta_{(i}^{p} d_{j m n)} .
$$

The triple product map $T(x, y, z)$ reads (up to contributions $\in \mathfrak{F}_{w}^{\perp}=\mathfrak{F} / \mathfrak{F}_{w}$ )

$$
T(x, y, z)_{M}=\frac{\partial I_{4}(x, y, z, w)}{\partial w^{M}}=K_{M N P Q} x^{N} y^{P} z^{Q},
$$

where the capital Latin indices span the entire FTS $\mathfrak{F}$, and $K_{M N P Q}=K_{(M N P Q)}$ is the rank-4 completely symmetric tensor characterizing $\mathfrak{F}[14,27,68]$. Note that, from its very definition eq. (8.9), $T(x, y, z)$ is completely symmetric in all its arguments [14].

\footnotetext{
${ }^{18}$ Recall that $d_{i j k}=d_{(i j k)}$ and $d^{i j k}=d^{(i j k)}$ throughout.

${ }^{19}$ At least in all reduced FTS's, Aut $\left(\mathfrak{F}\left(J_{3}\right)\right)$ is "of type $E_{7}$ " [14], and the ring of invariant polynomials is one-dimensional, and finitely generated (i.e., with no syzygies) by $I_{4}[71]$.
} 
Then, by using $\Omega$ to raise the symplectic indices, one can compute

$$
T(x, y, z)^{M}=\Omega^{M N} T(x, y, z)_{N} .
$$

By direct computation, one gets

$$
\begin{aligned}
& \frac{\partial I_{4}(x)}{\partial p^{0}}=-2\left(p^{0} q_{0}+p^{i} q_{i}\right) q_{0}-\frac{2}{3} d^{i j k} q_{i} q_{j} q_{k} ; \\
& \frac{\partial I_{4}(x)}{\partial p^{i}}=-2\left(p^{0} q_{0}+p^{j} q_{j}\right) q_{i}+2 q_{0} d_{i j k} p^{j} p^{k}+2 d_{i j k} d^{k l m} p^{j} q_{l} q_{m} ; \\
& \frac{\partial I_{4}(x)}{\partial q_{0}}=-2\left(p^{0} q_{0}+p^{i} q_{i}\right) p^{0}+\frac{2}{3} d_{i j k} p^{i} p^{j} p^{k} ; \\
& \frac{\partial I_{4}(x)}{\partial q_{i}}=-2\left(p^{0} q_{0}+p^{j} q_{j}\right) p^{i}-2 p^{0} d^{i j k} q_{j} q_{k}+d_{j k l} d^{l i n} p^{j} p^{k} q_{n} .
\end{aligned}
$$

In appendix D we present some explicit expressions for the triple product and other maps.

\subsection{The $T^{3}$ supergravity model and $\mathfrak{F}(\mathbb{R})$}

The so-called $T^{3}$ model of $\mathcal{N}=2, D=4$ supergravity is the smallest model in which the plane $\mathfrak{F}_{y \perp x}$ can be defined; such a model is comprised within all models based on (nondegenerate) reduced FTS's (cfr. e.g. (table 1$)$ ). In this model, it holds that $(i=1$, and $\left.p^{1} \equiv T\right)$

$$
\frac{1}{3 !} d_{i j k} p^{i} p^{j} p^{k}=T^{3} \Leftrightarrow d_{111}=6 .
$$

In the usual normalization of $d$-tensors used in supergravity literature ${ }^{20}$ it holds that (cfr. e.g. [73])

$$
d^{111}=\frac{2}{9} .
$$

In this case we have $(N=2, i=1, \operatorname{dim} \mathfrak{F}=4))$ :

$$
\begin{aligned}
x & =\left(p^{0}, p^{1}, q_{0}, q_{1}\right)^{T}, \\
I_{4}(x) & =\Delta(x)=-\left(p^{0} q_{0}+p^{1} q_{1}\right)^{2}+4 q_{0}\left(p^{1}\right)^{3}-\frac{4}{27} p^{0}\left(q_{1}\right)^{3}+\frac{4}{3}\left(p^{1} q_{1}\right)^{2} .
\end{aligned}
$$

By direct computation, one gets

$$
\begin{aligned}
& \frac{\partial I_{4}(x)}{\partial p^{0}}=-2\left(p^{0} q_{0}+p^{1} q_{1}\right) q_{0}-\frac{4}{27} q_{1}^{3} ; \\
& \frac{\partial I_{4}(x)}{\partial p^{1}}=-2\left(p^{0} q_{0}+p^{1} q_{1}\right) q_{1}+\frac{4}{9} q_{0}\left(p^{1}\right)^{2}+\frac{24}{9} p^{1} q_{1}^{2} ; \\
& \frac{\partial I_{4}(x)}{\partial q_{0}}=-2\left(p^{0} q_{0}+p^{1} q_{1}\right) p^{0}+4\left(p^{1}\right)^{3} ; \\
& \frac{\partial I_{4}(x)}{\partial q_{1}}=-2\left(p^{0} q_{0}+p^{1} q_{1}\right) p^{1}-\frac{4}{9} p^{0} q_{1}^{2}+\frac{12}{9}\left(p^{1}\right)^{2} q_{1} .
\end{aligned}
$$

which allows to compute the dual components by eq. (6.7).

\footnotetext{
${ }^{20}$ Which, however, is not the one used e.g. in [68].
} 
Since the $T^{3}$ model pertains to the unique reduced FTS for which $N=2$ (cfr. table 1 ), for this model $\operatorname{dim} \mathfrak{F}=2 N=4$ and the plane $\mathfrak{F}_{y \perp x}$ coincides with the whole space $\{$,$\} -$ orthogonal to the Freudenthal plane $\mathfrak{F}_{x}$ :

$$
N=2 \Rightarrow \mathfrak{F}_{y \perp x}=\mathfrak{F}_{x}^{\perp} .
$$

Thus, the FTS $\mathfrak{F}_{T^{3}} \equiv \mathfrak{F}(\mathbb{R})$, sitting in the spin- $\frac{3}{2}$ irrepr. 4 of $\operatorname{Aut}\left(\mathfrak{F}_{T^{3}}\right) \approx \operatorname{Conf}\left(J_{3}=\mathbb{R}\right)=$ $\mathrm{SL}(2, \mathbb{R})$, gets decomposed as follows:

$$
\mathfrak{F}_{T^{3}} \equiv \mathfrak{F}(\mathbb{R}) \equiv \begin{gathered}
\mathbf{4}=\mathbf{1}_{-3}+\mathbf{1}_{-1}+\mathbf{1}_{1}+\mathbf{1}_{3} \\
\operatorname{Conf}\left(J_{3}=\mathbb{R}\right)=\operatorname{SL}(2, \mathbb{R}) \longrightarrow \operatorname{SO}(1,1)_{K K}
\end{gathered},
$$

where $\mathrm{SO}(1,1)_{K K}$ is related to the radius of the $S^{1}$ in the dimensional reduction from minimal $(\mathcal{N}=2) D=5$ "pure" supergravity down to $D=4$ (giving rise to the $T^{3}$ model).

Let us start first with a particular configuration with $\Delta(x)<0$. Specifying (8.52) and (6.7)-(8.59) for the $T^{3}$ model, one has $\left(\epsilon=\operatorname{sgn}\left(x^{0} x_{0}\right)\right)$

$$
\begin{aligned}
& x=\left(x^{0}, 0, x_{0}, 0\right)^{T} ; \\
& \widetilde{x}=\epsilon\left(-x^{0}, 0, x_{0}, 0\right)^{T},
\end{aligned}
$$

with

$$
\Delta_{0} \equiv \Delta(x)=\Delta(\widetilde{x})=-\left(x^{0} x_{0}\right)^{2}<0 .
$$

Then, for a generic GFT transformation on $x$

$$
x_{F}=a x+b \widetilde{x} \in \mathfrak{F}_{x},
$$

it holds that

$$
\Delta\left(x_{F}\right)=-\left(a^{2}-b^{2}\right)^{2}\left(x^{0} x_{0}\right)^{2}=\left(a^{2}-b^{2}\right)^{2} \Delta(x)<0,
$$

and therefore $\mathfrak{F}_{x}$ lies completely in the rank- $4 \Delta<0$ orbit of Aut $\left(\mathfrak{F}\left(J_{3}=\mathbb{R}\right)\right)$.

Analogously, specifying eq. (8.63) and eq. (8.64) for the $T^{3}$ model, one obtains

$$
y=\left(0, y^{1}, 0, y_{1}\right)^{T}
$$

and, according to eq. (8.18),

$$
\Delta(y)=\frac{1}{3}\left(y^{1} y_{1}\right)^{2}>0,
$$

where the strict inequality holds, because we assume $y$ to be of maximal $(=4) \operatorname{rank}$ in $\mathfrak{F}_{T^{3}}$. Note that, while $x$ and $\widetilde{x}$ lie in the $\Delta<0$ orbit of $\operatorname{Aut}(\mathfrak{F}(\mathbb{R})), y$ belongs to the other rank- 4 orbit. $^{21}$

Starting from the decomposition eq. (8.24), the Freudenthal plane $\mathfrak{F}_{x}$ related to $x$ eq. (8.25) and the $\{$,$\} -orthogonal plane \mathfrak{F}_{y \perp x}=\mathfrak{F}_{x}^{\perp}$ can respectively be identified as follows:

$$
\begin{aligned}
\mathfrak{F}_{x} & =\underset{x^{0}}{D 6} \oplus \underset{x_{0}}{D 0}=\mathbf{1}_{-3}+\mathbf{1}_{3} ; \\
\mathfrak{F}_{y \perp x} & =\mathfrak{F}_{x}^{\perp}=\underset{y^{1}}{D 4} \oplus \underset{y_{1}}{D 2}=\mathbf{1}_{-1}+\mathbf{1}_{1} .
\end{aligned}
$$

\footnotetext{
${ }^{21}$ As pointed out above, there is a unique $\Delta>0$ orbit in the $T^{3}$ model.
} 
Nicely, within the interpretation of $\mathrm{SO}(1,1)_{K K}$ as a non-compact analogue of $D=4$ helicity of a would-be spin- $\frac{3}{2}$ (Rarita-Schwinger) particle, the Freudenthal plane $\mathfrak{F}_{x}$ pertains to the two massless helicity modes.

Let us recall that, while $\mathfrak{F}_{x}$ eq. (8.31) is a quadratic sub-FTS of $\mathfrak{F}_{T^{3}}$ (as discussed in section 8.1), $\mathfrak{F}_{y \perp x}=\mathfrak{F}_{x}^{\perp}$ eq. (8.32) is not a sub-FTS of $\mathfrak{F}$ (as discussed in section 5). This can be explicitly checked by relying on the treatment of subsection 8.2 ; in fact, for the $T^{3}$ model, $\mathfrak{F}_{y \perp x}=\mathfrak{F}_{x}^{\perp}$ (8.32) is not closed under $T$. Out of the four cases 1-4 listed at the end of subsection 8.2, only the last one (4) is to be considered: in this case, the condition of closure of $\mathfrak{F}_{y \perp x}=\mathfrak{F}_{x}^{\perp}$ eq. (8.32) under $T$ is that $y^{1}$ is $\operatorname{rank}<3$ in $J_{3}=\mathbb{R}$ and $y_{1}$ is rank $<3$ in $\bar{J}_{3}=\mathbb{R}$, namely

$$
y_{1}=0=y^{1} \Leftrightarrow y=0 \in \mathfrak{F}
$$

Thus, the condition of closure of $\mathfrak{F}_{y \perp x}=\mathfrak{F}_{x}^{\perp}$ eq. (8.32) under $T$ implies, in the case of the $T^{3}$ model, an absurdum, namely that the rank-4 element $y \in \mathfrak{F}_{x}^{\perp}$ be the null element of the FTS $\mathfrak{F}_{T^{3}} \equiv \mathfrak{F}(\mathbb{R})$. Therefore, $\mathfrak{F}_{y \perp x}=\mathfrak{F}_{x}^{\perp}$ eq. (8.32) is not closed under $T$.

In other words, as also pointed out above, in order for $y=\left(0, y^{1}, 0, y_{1}\right)^{T} \in \mathfrak{F}_{x}^{\perp}$ to be rank-4 (as assumed throughout), it must have both components non-vanishing; from eq. (8.30) one can observe that in the $T^{3}$ model $y$ belongs to the rank-4, $\Delta>0, \operatorname{Aut}\left(\mathfrak{F}_{T^{3}}\right)=$ $\mathrm{SL}(2, \mathbb{R})$ orbit, unless $y^{1}=0$ and/or $y_{1}=0$, in which case it has $\operatorname{rank}<4$. Therefore an element $y$ of the form

$$
y=\left(0, y^{1}, 0, y_{1}\right)^{T} \in \mathfrak{F}_{x}^{\perp}
$$

is rank-4 (and necessarily in the unique $\Delta>0$ orbit) iff $y^{1} \neq 0$ and $y_{1} \neq 0$.

Furthermore, we are interested in the behaviour of the quartic invariant $\Delta$ on the $D 4 \oplus D 2 \mathfrak{F}_{y \perp x}$ plane. General results are presented in section B, in particular in eq. (4.23) and eq. (4.24) which can be used here. According to these results ${ }^{22}$

$$
\Delta\left(\Upsilon_{x}(y)\right)=\Delta(x)^{2} \Delta(y)
$$

The sign of $\Delta\left(\Upsilon_{x}(y)\right)$ depends only on the sign of $\Delta(y)$ implying that $\Upsilon_{x}(y)$ belongs to the same rank-4 $(\Delta>0) \operatorname{Aut}(\mathfrak{F}(\mathbb{R}))$-orbit as $y$. Explicitly in this case

$$
\Delta\left(\Upsilon_{x}(y)\right)=\Delta(x)^{2} \Delta(y)=\frac{1}{3}\left(x^{0} x_{0}\right)^{4}\left(y^{1} y_{1}\right)^{2}>0
$$

For a generic element $r$

$$
r=a y+b \Upsilon_{x}(y) \in \mathfrak{F}_{y \perp x}=\mathfrak{F}_{x}^{\perp} \quad(a, b \in \mathbb{R}),
$$

one gets eq. (4.23) and eq. (4.24)

$$
\Delta(r)=\left(a^{2}-|\Delta(x)| b^{2}\right)^{2} \Delta(y) \geqslant 0 .
$$

${ }^{22}$ We can explicitly write the $\Upsilon$ map (see eq. (2.6))

$$
\left(\Upsilon_{x}(y)\right)^{0}=0 ;\left(\Upsilon_{x}(y)\right)^{1}=\frac{1}{2} x^{0} x_{0} y^{1} ;\left(\Upsilon_{x}(y)\right)_{0}=0 ;\left(\Upsilon_{x}(y)\right)_{1}=-\frac{1}{2} x^{0} x_{0} y_{1}
$$


Thus, following the general behaviour explained in appendix $\mathrm{B}, r \in \mathfrak{F}_{y \perp x}$ is not of the same $($ maximal $=4)$ rank orbit as $y\left(\right.$ and $\left.\Upsilon_{x}(y)\right)$ only when

$$
\Delta(r)=0 \Leftrightarrow a^{2}-\left(x^{0} x_{0}\right)^{2} b^{2}=0 \Leftrightarrow a^{2}=|\Delta(x)| b^{2} .
$$

The conditions for $r \in \mathfrak{F}_{y \perp x} \subset \mathfrak{F}_{x}^{\perp}$ to lie in the rank-3, rank-2 or even rank-1 orbits might be easily studied using expressions eq. (4.23) and eq. (4.24)).

Let us study now the family of configurations with $D 4-D 0$ charges. This family includes configurations with both $\Delta(x)>0$ and $\Delta(x)<0$ possibilities. Let us take, ${ }^{23}$

$$
x=\left(0, x^{1}, x_{0}, 0\right)^{T},
$$

then one obtains

$$
\Delta(x)=\Delta(\widetilde{x})=4 x_{0}\left(x^{1}\right)^{3} .
$$

Thus the sign of $\Delta(x)$ equals the sign of $x_{0} x^{1}$ :

$$
\operatorname{sgn} \Delta(x)=\operatorname{sgn}\left(x_{0} x^{1}\right) .
$$

For a positive sign, $\operatorname{sgn}\left(x_{0} x^{1}\right)>0$, the dual is a $D 6-D 2$ configuration, it reads

$$
\widetilde{x}=\frac{1}{\sqrt{\Delta_{0}}}\left(-\left(x^{1}\right)^{3}, 0,0,3 x_{0}\left(x^{1}\right)^{2}\right)
$$

$x$ and $\widetilde{x}$ belong to the same (rank- $4, \Delta>0$ ) orbit of $\operatorname{Aut}\left(\left(\mathfrak{F}=J_{3}\right)\right.$ ), which is unique in this model (cfr. [75], and refs. therein). For a generic element $x_{F}=a x+b \widetilde{x} \in \mathfrak{F}_{x}$, it holds that

$$
\Delta\left(x_{F}\right)=\left(a^{2}+b^{2}\right)^{2} \Delta(x)>0,
$$

implying that $\mathfrak{F}_{x}$ lies completely in the unique rank- $4 \Delta>0$ orbit of $\operatorname{Aut}(\mathfrak{F}(\mathbb{R}))$.

Then, let us pick a rank-4 element $y \in \mathfrak{F}_{x}^{\perp}$, that means which is $\{$,$\} -orthogonal to x$ and $\widetilde{x}$, one can show that the most general element of this kind is given by the charge configuration:

$$
y=\left(\frac{x^{1}}{x_{0}} y_{1}, y^{1},-3 \frac{x_{0}}{x^{1}} y^{1}, y_{1}\right)
$$

whose quartic invariant is given by

$$
\begin{aligned}
\Delta(y) & =-\frac{8}{3}\left(y^{1}\right)^{2} y_{1}^{2}-12 \frac{x_{0}}{x^{1}}\left(y^{1}\right)^{4}-\frac{4}{27} \frac{x^{1}}{x_{0}} y_{1}^{4}, \\
& =-\frac{4}{27\left(x_{0} x^{1}\right)}\left(9 x_{0}\left(y^{1}\right)^{2}+x^{1}\left(y_{1}\right)^{2}\right)^{2}
\end{aligned}
$$

thus the signs of $\Delta(x)$ and $\Delta(y)$ are opposite

$$
\operatorname{sgn} \Delta(y)=-\operatorname{sgn}\left(x_{0} x^{1}\right)=-\operatorname{sgn} \Delta(x) .
$$

\footnotetext{
${ }^{23}$ This can be seen as an special case of eq. (8.81) and eqs. (8.83)-(8.85) for the $T^{3}$ model.
} 
In the case of $\left(x_{0} x^{1}\right)=0$ then $\mathfrak{F}_{x}^{\perp} \sim D 6 \oplus D 4$ (for $\left.x_{0}=0\right)$ and $\mathfrak{F}_{x}^{\perp} \sim D 2 \oplus D 4$ (for $x^{1}=0$ ) Moreover, according to eq. (4.23) and eq. (4.24)

$$
\Delta\left(\Upsilon_{x}(y)\right)=\Delta(x)^{2} \Delta(y) .
$$

The sign of $\Delta\left(\Upsilon_{x}(y)\right)$ depends only on the sign of $\Delta(y)$. Both of them are negative in our current case. For a generic element $r$

$$
r=a y+b \Upsilon_{x}(y) \in \mathfrak{F}_{y \perp x}=\mathfrak{F}_{x}^{\perp}
$$

$(a, b \in \mathbb{R})$, one gets eq. (4.23) and eq. (4.24)

$$
\Delta(r)=\left(a^{2}+\Delta(x) b^{2}\right)^{2} \Delta(y) .
$$

implying that $\Upsilon_{x}(y)$ and for the case any $\Upsilon_{x}(r)$ lies in the same (maximal rank) Aut $(\mathfrak{F}(\mathbb{R})$ )orbit as $y .{ }^{24}$

\subsection{General $D 0-D 6 / D 2-D 4$ sectors}

Let us consider a particular configuration with only $D 0-D 6$ charges with an arbitrary number of them. We start by identifying $x$ with the rank-4, strictly regular element of the FTS $\mathfrak{F}$ given by the $D 0-D 6$ brane charge configuration

$$
x_{D 0 D 6} \equiv\left(p^{0}, 0^{i}, q_{0}, 0_{i}\right)^{T} \in \mathfrak{F},
$$

for any element of this configuration we have ${ }^{25}$

$$
\Delta\left(x_{D 0 D 6}\right)=-\left(p^{0} q_{0}\right)^{2}<0 .
$$

One can compute the Freudenthal dual $\widetilde{x}$. Using the expressions

$$
\begin{aligned}
& \frac{\partial I_{4}(x)}{\partial p^{0}}=-2\left(p^{0} q_{0}\right) q_{0}, \\
& \frac{\partial I_{4}(x)}{\partial p^{1}}=0, \\
& \frac{\partial I_{4}(x)}{\partial q_{0}}=-2\left(p^{0} q_{0}\right) p^{0}, \\
& \frac{\partial I_{4}(x)}{\partial q_{1}}=0,
\end{aligned}
$$

\footnotetext{
${ }^{24}$ Explicitly, from eq. (8.98), one obtains

$$
\left(\Upsilon_{x}(y)\right)^{0}=-6\left(x^{1}\right)^{2} y^{1} ;\left(\Upsilon_{x}(y)\right)^{1}=\frac{2}{3}\left(x^{1}\right)^{2} y_{1} ;\left(\Upsilon_{x}(y)\right)_{0}=-2 x_{0} x^{1} y_{1} ;\left(\Upsilon_{x}(y)\right)_{1}=-6 x_{0} x^{1} y^{1},
$$
}

yielding

$$
\Delta\left(\Upsilon_{x}(y)\right)=-\frac{4}{3} x_{0}^{2}\left(x^{1}\right)^{6}\left(y^{1}\right)^{2} y_{1}^{2}-6 x_{0}^{3}\left(x^{1}\right)^{5}\left(y^{1}\right)^{4}-\frac{2}{27} x_{0}\left(x^{1}\right)^{7} y_{1}^{4} .
$$

\footnotetext{
${ }^{25}$ This characterizes $\mathfrak{F}$ as a reduced [14] FTS.
} 
which allows to compute the dual components using eq. (6.7). We arrive to $\left(\epsilon=\operatorname{sgn}\left(p^{0} q_{0}\right)\right)$

$$
\begin{aligned}
\widetilde{x_{D 0 D 6}} & \equiv\left(\widetilde{p}^{0}, 0^{i}, \widetilde{q}_{0}, 0_{i}\right)^{T} \in \mathfrak{F}, \\
& =\epsilon\left(-p^{0}, 0^{i}, q_{0}, 0_{i}\right) .
\end{aligned}
$$

Thus, depending on the $\operatorname{sign}^{26}$ of $p^{0} q_{0}$. Note that

$$
\Delta(x)=\Delta(\widetilde{x})<0,
$$

and thus $\widetilde{x}$ belongs to the same (unique) rank- $4 \Delta<0$ orbit of $\operatorname{Aut}\left(\mathfrak{F}\left(J_{3}\right)\right)$ as $x$. Namely, when $p^{0} q_{0}>0$, the action of Freudenthal duality on $x_{D 0 D 6}$ amounts to flipping $p^{0}$ only, whereas when $p^{0} q_{0}<0$, the action of Freudenthal duality on $x_{D 0 D 6}$ amounts to flipping $q_{0}$ only.

Associated to a GFT transformation on $x$, one defines the Freudenthal plane $\mathfrak{F}_{x} \subset \mathfrak{F}$ $\left(\operatorname{dim} \mathfrak{F}_{x}=2\right)$, spanned by $x$ and $\widetilde{x}$, whose generic element is

$$
x_{F}=a x+b \widetilde{x} \in \mathfrak{F}_{x} \quad(a, b \in \mathbb{R}) .
$$

Within the choice above, $\mathfrak{F}_{x}$ is coordinatized by the charges of $D 0$ and $D 6$ branes, respectively being the electric and magnetic charges $x_{0}$ and $x^{0}$ of the KK Abelian vector in the reduction $D=5 \rightarrow 4$. In other words, within the position (which does not imply any loss of generality for reduced FTS's), the Freudenthal plane $\mathfrak{F}_{x}$ is spanned (in a canonical Darboux symplectic frame - see below -) by the electric and magnetic charges $x_{0}$ and $x^{0}$ of the $D=5 \rightarrow 4$ Kaluza-Klein Abelian vector (which is the $D=4$ graviphoton in the $\mathcal{N}=2$ supersymmetric interpretation).

Note that $\mathfrak{F}_{x}$ lies completely in the (unique) rank- $4 \Delta<0$ orbit of $\operatorname{Aut}\left(\mathfrak{F}\left(J_{3}\right)\right)$, because eq. (3.9)

$$
\begin{aligned}
\Delta\left(X_{F}\right) & =-\left(a x^{0}+b \widetilde{x}^{0}\right)^{2}\left(a x_{0}+b \widetilde{x}_{0}\right)^{2} \\
& =-\left(a^{2}-b^{2}\right)^{2}\left(x^{0} x_{0}\right)^{2}=\left(a^{2}-b^{2}\right)^{2} \Delta(x)<0 .
\end{aligned}
$$

This implies that $s$ belongs to the same maximal $(=4) \operatorname{rank}, \Delta<0 \operatorname{Aut}\left(\mathfrak{F}\left(J_{3}\right)\right)$-orbit as $x$ and $\widetilde{x}$, unless $a^{2}=b^{2}$. This observation actually yields interesting consequences for multi-centered black hole physics, as briefly discussed in section 10 .

The $D 2-D 4$ orthogonal space $\mathfrak{F}_{x}^{\perp}$, and the plane $\mathfrak{F}_{y \perp x} \subset \mathfrak{F}_{x}^{\perp}$. One can choose a rank-4 element $y \in \mathfrak{F}$ which is $\{$,$\} -orthogonal to the generic D 0-D 6$ element $x$ defined

\footnotetext{
${ }^{26}$ The result eq. (8.59) defines a $\mathbb{Z}_{4}$ symmetry in the 2 -dim. Freudenthal plane $\mathfrak{F}_{x} \subset \mathfrak{F}$, spanned by $x_{D 0 D 6}$ eq. (8.52) and its Freudenthal dual $\widehat{x_{D 0 D 6}}$ (8.59) (or, equivalently, in the Darboux canonical basis, by the magnetic and electric charges $p^{0}$ and $q_{0}$ of the $5 D \rightarrow 4 D$ KK Abelian vector — see below - ): e.g., starting from $p^{0}$ and $q_{0}$ both positive (denoted by " $(+,+)$ "), the iteration of Freudenthal duality yields

$$
(+,+) \stackrel{\sim}{\rightarrow}(-,+) \stackrel{\sim}{\rightarrow}(-,-) \stackrel{\sim}{\rightarrow}(+,-) \stackrel{\sim}{\rightarrow}(+,+) .
$$

This provides a simple realisation of the $\mathbb{Z}_{4}$ symmetry characterizing every Freudenthal plane, as a consequence of the anti-involutivity of Freudenthal duality itself.
} 
before and its dual and $\widetilde{x}$. A possible, particular, choice is provided by a $D 2-D 4$ brane charge configuration:

$$
\begin{aligned}
y & \equiv\left(0, y^{i}, 0, y_{i}\right)^{T} \in \mathfrak{F}, \\
\Delta(y) & =-\left(y^{i} y_{i}\right)^{2}+d_{i j k} d^{i l m} y^{j} y^{k} y_{l} y_{m} \gtrless 0 .
\end{aligned}
$$

In eq. (8.64), the case of vanishing $\Delta$ has been excluded because $y$ is chosen to be of maximal $(=4)$ rank in $\mathfrak{F}$. By recalling $(8.2)$, one can immediately check that $\left(\operatorname{dim} \mathfrak{F}_{x}^{\perp}=\right.$ $2 N-2)$

$$
\{x, y\}=0=\{\widetilde{x}, y\} \Leftrightarrow y \in \mathfrak{F}_{x}^{\perp} \equiv \mathfrak{F} / \mathfrak{F}_{x} .
$$

One can compute the components of $\Upsilon_{x}(y)^{M}$ eq. (2.6) as given by

$$
\begin{aligned}
& \left(\Upsilon_{x}(y)\right)^{0}=0 ; \\
& \left(\Upsilon_{x}(y)\right)^{i}=x^{0} x_{0} y^{i} ; \\
& \left(\Upsilon_{x}(y)\right)_{0}=0 ; \\
& \left(\Upsilon_{x}(y)\right)_{i}=-x^{0} x_{0} y_{i} ;
\end{aligned}
$$

thus, $\Upsilon_{x}(y)$ is still given by a rank- $4 D 2-D 4$ brane charge configuration, and it holds that

$$
\left\{x, \Upsilon_{x}(y)\right\}=0=\left\{\widetilde{x}, \Upsilon_{x}(y)\right\} \Leftrightarrow \Upsilon_{x}(y) \in \mathfrak{F}_{x}^{\perp} \equiv \mathfrak{F} / \mathfrak{F}_{x} .
$$

Consequently, one can define the 2-dim. plane $\mathfrak{F}_{y \perp x} \subset \mathfrak{F}_{x}^{\perp}$, spanned by $y$ and $\Upsilon_{x}(y)$, whose generic element is $r=a y+b \Upsilon_{x}(y) \in \mathfrak{F}_{y \perp x}(a, b \in \mathbb{R}$, in our classical/supergravity treatment. In particular, note that $\Upsilon_{x}(y)$ belongs to the same $\operatorname{Aut}\left(\mathfrak{F}\left(J_{3}\right)\right)$-orbit as $y$, because (consistent with the general eq. (4.23) and eq. (4.24)), it holds that

$$
\Delta\left(\Upsilon_{x}(y)\right)=\left(x^{0} x_{0}\right)^{4} \Delta(y)=(\Delta(x))^{2} \Delta(y) \gtrless 0 .
$$

whose sign depends only on the sign of $\Delta(y)$.

It is worth remarking that $\Upsilon_{x}(y)$ automatically satisfies eq. (8.70) for every pair $y^{i}$ and $y_{i}$, with $i=1, \ldots, N-1$. In fact, regardless of $d_{i j k}$ and $d^{i j k}$, when only a pair $y^{i}$ and $y_{i}$ for a fixed $i$ is non-vanishing (among all $y^{i}$ 's and $y_{i}^{\prime}$ 's), then $y$ is non-trivially of rank-4 in $\mathfrak{F}$, because generally $\Delta(y) \neq 0$, since at least the term $-\left(y^{i} y_{i}\right)^{2}$ is present (cfr. eqs. (8.3)-(8.4)). Therefore, one can define $N-1$ distinct planes $\left(\mathfrak{F}_{y \perp x}^{\perp}\right)_{i}$ 's, orthogonal to the Freudenthal plane $\mathfrak{F}_{x}$, cfr. subsection 8.3.

Moreover, note that $\mathfrak{F}_{y \perp x}$ intersects at least three orbits of Aut(F). Indeed, it holds that, using eq. (4.23) and eq. (4.24), $\left(c \equiv x^{0} x_{0} b\right)$

$$
\begin{aligned}
\Delta(r) & =(a+c)^{2}(a-c)^{2} \Delta(y)=\left(a^{2}-c^{2}\right)^{2} \Delta(y) \\
& =\left(a^{2}-|\Delta(x)| b^{2}\right)^{2} \Delta(y) \gtreqless 0,
\end{aligned}
$$

which implies $r \in \mathfrak{F}_{y \perp x} \subset \mathfrak{F}_{x}^{\perp}$ to be not of the same (maximal $=4$ ) rank as $y$ (and $\Upsilon_{x}(y)$ ) only when (recall eq. (8.64))

$$
\Delta(r)=0 \Leftrightarrow a^{2}-c^{2}=0 \rightarrow a^{2}-\left(x^{0} x_{0}\right)^{2} b^{2}=0 .
$$

The conditions for $r \in \mathfrak{F}_{y \perp x} \subset \mathfrak{F}_{x}^{\perp}$ to lie in the rank-3, rank-2 or even rank-1 orbits may be easily inferred. 
Closure of the $\mathbf{D 2}-\boldsymbol{D} \mathbf{4} \mathfrak{F}_{\boldsymbol{y} \perp \boldsymbol{x}}$ under $\boldsymbol{T}$. The plane $\mathfrak{F}_{y \perp x}$ is not generally closed under the triple map $T$ (or, equivalently, under Freudenthal duality $\sim$ ), see section 3 .

Within the framework under consideration, namely within the $4 D / 5 D$ special coordinates' symplectic frame of reduced FTS's and within the choice given by eq. (8.63) of the rank-4 element $x \in \mathfrak{F}$ (with $\Delta(x)<0$ ) and of the rank-4 element $y \in \mathfrak{F}_{x}^{\perp}=\mathfrak{F} / \mathfrak{F}_{x}$ (with $\Delta(y) \gtrless 0$ ), we study now more in detail the condition of closure of the plane $\mathfrak{F}_{y \perp x}$ under $T$.

In order to determine the condition of closure of $\mathfrak{F}_{y \perp x}$ under $T$, we have to explicitly compute $T(r) \equiv T(r, r, r)$ for a generic element $r=a y+b \Upsilon_{x}(y) \in \mathfrak{F}_{y \perp x}$, and for any $D 2-D 4$ configuration $y$. This is given by, (see appendix D) $\left(c \equiv x^{0} x_{0} b, T(y)=T(y, y, y)\right)$.

$$
T(r)_{M}:\left\{\begin{array}{l}
T(r)_{0}=(a-c)^{3} T(y)_{0} \\
T(r)_{i}=\left(a^{2}-c^{2}\right)(a-c) T(y)_{i} \\
T(r)^{0}=(a+c)^{3} T(y)^{0} \\
T(r)^{i}=\left(a^{2}-c^{2}\right)(a+c) T(y)^{i}
\end{array}\right.
$$

Then $T(r)^{M}=\Omega^{M N} T(r)_{N}$. Thus, the plane $\mathfrak{F}_{y \perp x}$ is closed under $T$ iff

$$
T(r)^{0}=0=T(r)_{0} \Leftrightarrow\left\{\begin{array}{l}
(a-c)^{3} d^{i j k} y_{i} y_{j} y_{k}=0 ; \\
(a+c)^{3} d_{i j k} y^{i} y^{j} y^{k}=0 .
\end{array}\right.
$$

There are various cases, as follows:

1. $y^{i}$ is rank-3 in $J_{3}$ and $y_{i}$ is rank-3 in $\bar{J}_{3}$, namely

$$
d^{i j k} y_{i} y_{j} y_{k} \neq 0, d_{i j k} y^{i} y^{j} y^{k} \neq 0
$$

In this case, no solutions exist to the system eq. (8.76), and $\mathfrak{F}_{y \perp x}$ is not closed under $T$.

2. $y^{i}$ is rank $<3$ in $J_{3}$ and $y_{i}$ is rank-3 in $\bar{J}_{3}$, namely

$$
d^{i j k} y_{i} y_{j} y_{k} \neq 0, d_{i j k} y^{i} y^{j} y^{k}=0
$$

In this case, $T(r)_{0}=0$ is automatically satisfied, while $T(r)^{0}=0$ has solution $a=x^{0} x_{0} b$. However, for a fixed $x$, this solution is a line in $\mathbb{F}^{2}=\mathbb{R}^{2}$ spanned by $(a, b)$, and thus is codimension- 1 in $\mathfrak{F}_{y \perp x}$. Therefore, only the $x$-dependent 1 -dimensional locus $a=x^{0} x_{0} b$ in $\mathfrak{F}_{y \perp x} \subset \mathfrak{F}_{x}^{\perp}$, and not $\mathfrak{F}_{y \perp x}$ itself, is closed under $T$.

3. $y^{i}$ is rank-3 in $J_{3}$ and $y_{i}$ is $\operatorname{rank}<3$ in $\bar{J}_{3}$, namely

$$
d^{i j k} y_{i} y_{j} y_{k}=0, d_{i j k} y^{i} y^{j} y^{k} \neq 0 .
$$

In this case, $T(r)^{0}=0$ is automatically satisfied, while $T(r)_{0}=0$ has solution $a=$ $-x^{0} x_{0} b$. However, for a fixed $x$, this solution is a line in $\mathbb{F}^{2}=\mathbb{R}^{2}$ spanned by $(a, b)$, and thus is codimension- 1 in $F_{y \perp x}$. Therefore, only the $x$-dependent 1 -dimensional locus $a=-x^{0} x_{0} b$ in $F_{y \perp x} \subset F_{x}^{\perp}$, and not $F_{y \perp x}$ itself, is closed under $T$. 
4. $y^{i}$ is rank $<3$ in $J_{3}$ and $y_{i}$ is rank $<3$ in $\bar{J}_{3}$, namely

$$
d^{i j k} y_{i} y_{j} y_{k}=0=d_{i j k} y^{i} y^{j} y^{k} .
$$

In this case, the system eq. (8.76) is automatically satisfied $\forall a, b \in \mathbb{R}$, and $\mathfrak{F}_{y \perp x}$ is therefore $\operatorname{closed}^{27}$ under $T$. Note that the condition eq. (8.80) is not inconsistent with the assumption of $y$ (8.63) to be a rank- 4 element of $\mathfrak{F}$. In fact, if both $y^{i}$ and $y_{i}$ are rank-2 elements in $J_{3}$ resp. $\bar{J}_{3}$, then $\Delta(y)$ eq. (8.64) is still generally non-vanishing, with the second term vanishing iff $d_{i j k} d^{i l m} y^{j} y^{k} y_{l} y_{m}=0$ (in this latter case, when non-vanishing, $\Delta(y)<0$, and $y$ - and $\Upsilon_{x}(y)$ as well - would lie in the same $\Delta<0$ $\operatorname{Aut}\left(\mathfrak{F}\left(J_{3}\right)\right)$-orbit as $x$ and $\left.\widetilde{x}\right)$. On the other hand, if $y^{i}$ and/or $y_{i}$ are rank- 1 elements in $J_{3}$ resp. $\bar{J}_{3}$, still $y$ can be a rank-4 element of $\mathfrak{F}\left(J_{3}\right)$, because $\Delta(y)=-\left(y^{i} y_{i}\right)^{2} \leqslant 0$ in this case, and thus (when the inequality strictly holds), $y$ - and $\Upsilon_{x}(y)$ as well would lie, as above, in the same $\Delta<0 \operatorname{Aut}\left(\mathfrak{F}\left(J_{3}\right)\right)$-orbit as $x$ and $\widetilde{x}$.

\subsection{The general $D 0-D 4$ sector}

The Freudenthal plane $\mathfrak{F}_{x}$. We start by identifying $x$ with the rank-4 element of the FTS $\mathfrak{F}$ given by the ${ }^{28} D 0-D 4$ brane charge configuration:

$$
x_{D 0 D 4} \equiv\left(0, x^{i}, x_{0}, 0_{i}\right)^{T} \in \mathfrak{F},
$$

and we further impose that $x_{D 0 D 4}$ belongs to (one of) the $\Delta>0 \operatorname{Aut}(\mathfrak{F})$-orbit(s) (see section 8.4).

$$
\Delta\left(x_{D 0 D 4}\right)=\frac{2}{3} x_{0} d_{i j k} x^{i} x^{j} x^{k}>0 .
$$

From the definition eq. (6.7) (note that $\epsilon=1$ in this case), one can compute that the Freudenthal-dual $\widetilde{x_{D 0 D 4}}$ of the $D 0-D 4$ configuration eq. (8.81) is a $D 2-D 6$ configuration, namely ${ }^{29}$ The dual is

$$
\widetilde{x} \equiv\left(\widetilde{x}^{0}, 0^{i}, 0, \widetilde{x}_{i}\right)^{T} \in \mathfrak{F}
$$

with eq. (6.7)

$$
\begin{aligned}
& \widetilde{x}^{0}=-\frac{1}{3 \sqrt{\Delta(x)}} d_{j k l} x^{j} x^{k} x^{l} ; \\
& \widetilde{x}_{i}=\frac{1}{\sqrt{\Delta(x)}} x_{0} d_{i j k} x^{j} x^{k} .
\end{aligned}
$$

By exploiting the adjoint identity (see [46, 73, 74] and refs. therein) of the Jordan algebra $J_{3}$ underlying the reduced FTS $\mathfrak{F}$ eq. (8.8) one can also check that $\Delta$ is invariant under Freudenthal duality:

$$
\Delta\left(x_{D 0 D 4}\right)=\Delta\left(\widetilde{x_{D 0 D 4}}\right)>0,
$$

and thus that $\widetilde{x_{D 0 D 4}}$ would lies in the same $\operatorname{Aut}\left(\mathfrak{F}\left(J_{3}\right)\right)$-orbit as $x_{D 0 D 4}$.

\footnotetext{
${ }^{27}$ It could have also characterized as a 2 -dimensional sub-FTS of $\mathfrak{F}$.

${ }^{28}$ We might have as well started with a $D 2-D 6$ configuration, and perform an equivalent treatment (obtaining, as evident from the treatment given below and from the anti-involutivity of Freudenthal duality, a $D 0-D 4$ configuration as Freudenthal-dual of the starting $D 2-D 6$ configuration).

${ }^{29}$ This has been computed for the $S T U$ model in Example 1 of section V.A of [17] (cfr. (198)-(200) therein); see also the treatment of section 8.4.
} 
Thus, one can define the Freudenthal plane $\mathfrak{F}_{x}\left(\operatorname{dim} \mathfrak{F}_{x}=2\right)$, spanned by $x$ eq. (8.81) and $\widetilde{x}$ (8.83), whose generic element is $x_{F}=a x+b \widetilde{x} \in \mathfrak{F}_{x}, a, b \in \mathbb{R}$. By using eq. (8.8) again, one can also compute that

$$
\Delta\left(x_{F}\right)=\left(a^{2}+b^{2}\right)^{2} \Delta(x)>0,
$$

implying that $\mathfrak{F}_{x}$ lies completely in the rank- $4 \Delta>0 \operatorname{orbit}^{30}$ of $\operatorname{Aut}\left(\mathfrak{F}\left(J_{3}\right)\right)$.

The orthogonal space $\mathfrak{F}_{x}^{\perp}$, and the plane $\mathfrak{F}_{\boldsymbol{y} \perp \boldsymbol{x}} \subset \mathfrak{F}_{x}^{\perp}$. Then, one can pick another rank-4 element $y \in \mathfrak{F}$ which is $\{$,$\} -orthogonal to x$ eq. (8.81) and $\widetilde{x}$ (8.83); the most general element of this kind is given by the charge configuration:

$$
y \equiv\left(y^{0}, y^{i}, y_{0}, y_{i}\right)^{T} \in \mathfrak{F},
$$

constrained

$$
\{x, y\}=0=\{\widetilde{x}, y\} \Leftrightarrow\left\{\begin{array}{l}
y^{0}=\frac{1}{x_{0}} x^{i} y_{i} \\
y_{0}=-3 x_{0} \frac{d_{i j k} y^{i} x^{j} x^{k}}{d_{l m n} x^{l} x^{m} x^{n}} .
\end{array}\right.
$$

One can also compute that

$$
\begin{aligned}
\Delta(y)= & -\left(-3 \frac{d_{k l m} y^{k} x^{l} x^{m}}{d_{q r s} x^{q} x^{r} x^{s}} x^{j} y_{j}+y^{j} y_{j}\right)^{2}-2 x_{0} \frac{d_{k l m} y^{k} x^{l} x^{m}}{d_{q r s} x^{q} x^{r} x^{s}} d_{i j t} y^{j} y^{j} y^{t} \\
& -\frac{2}{3} \frac{1}{x_{0}} x^{j} y_{j} d^{k l m} y_{k} y_{l} y_{m}+d_{i j k} d^{i l m} y^{j} y^{k} y_{l} y_{m} \gtrless 0 .
\end{aligned}
$$

In eq. (8.90), the case of vanishing $\Delta$ has been excluded because $y$ is chosen to be of rank- 4 in $\mathfrak{F}$.

One can compute the components of $\Upsilon_{x}(y)^{M}$ eq. (2.6) as given by

$$
\begin{aligned}
& \left(\Upsilon_{x}(y)\right)^{0}=-d_{i j k} x^{i} x^{j} y^{k} \\
& \left(\Upsilon_{x}(y)\right)^{i}=-d_{k l m} d^{m i j} x^{k} x^{l} y_{j}+2 x^{j} y_{j} x^{i} \\
& \left(\Upsilon_{x}(y)\right)_{0}=-2 x^{j} y_{j} x_{0} ; \\
& \left(\Upsilon_{x}(y)\right)_{i}=2 x_{0} d_{i j k} x^{j} y^{k}-3 x_{0} \frac{d_{k l m} y^{k} x^{l} x^{m}}{d_{\text {qrs }} x^{q} x^{r} x^{s}} d_{i j t} x^{j} x^{t},
\end{aligned}
$$

which for this case can be written as

$$
\begin{aligned}
& \left(\Upsilon_{x}(y)\right)^{0}=-d_{i j k} x^{i} x^{j} y^{k} ; \\
& \left(\Upsilon_{x}(y)\right)^{i}=-d_{k l m} d^{m i j} x^{k} x^{l} y_{j}+y^{0} x_{0} x^{i} ; \\
& \left(\Upsilon_{x}(y)\right)_{0}=-2 y^{0} x_{0}^{2} ; \\
& \left(\Upsilon_{x}(y)\right)_{i}=2 x_{0} d_{i j k} x^{j} y^{k}+y_{0} d_{i j t} x^{j} x^{t} .
\end{aligned}
$$

By exploiting (8.8), one can then check that $\Upsilon_{x}(y)$ eq. (8.98) automatically satisfies

$$
\left\{x, \Upsilon_{x}(y)\right\}=0=\left\{\widetilde{x}, \Upsilon_{x}(y)\right\} \Leftrightarrow \Upsilon_{x}(y) \in \mathfrak{F}_{x}^{\perp} \equiv \mathfrak{F} / \mathfrak{F}_{x},
$$

\footnotetext{
${ }^{30}$ Again, as pointed out above, some subtleties may arise, related to the splitting of the $\Delta>0$ locus of $\mathfrak{F}$ (cfr. section 8.4, and e.g. [50, 75, 76], and refs. therein).
} 
for every pair $y^{i}$ and $y_{i}$, with $i=1, \ldots, N-1$. In fact, regardless of $d_{i j k}$ and $d^{i j k}$, when only a pair $y^{i}$ and $y_{i}$ for a fixed $i$ is non-vanishing (among all $y^{i}$ 's and $y_{i}$ 's), then $y$ is non-trivially of rank- 4 in $\mathfrak{F}$, because generally $\Delta(y) \neq 0$, since at least the term $-\left(y^{i} y_{i}\right)^{2}$ is present (cfr. eqs. (8.3)-(8.4)). Therefore, one can define $N-1$ distinct planes $\left(\mathfrak{F}_{y \perp x}^{\perp}\right)_{i}$ 's, orthogonal to the Freudenthal plane $\mathfrak{F}_{x}$, cfr. subsection 8.3.

Moreover, (consistently with eq. (4.23) and eq. (4.24)) one can compute that

$$
\begin{aligned}
\Delta\left(\Upsilon_{x}(y)\right) & =-\frac{8}{9} x_{0}^{2}\left(d_{a b c} x^{a} x^{b} x^{c}\right)^{2}\left[\begin{array}{l}
\frac{1}{2}\left(-3 \frac{d_{k l m} y^{k} x^{l} x^{m}}{d_{q r s} x^{q} x^{x} x^{s}} x^{j} y_{j}+y^{j} y_{j}\right)^{2}+x_{0} \frac{d_{k l m} y^{k} x^{l} x^{m}}{d_{q r s} x^{q} x^{r} x^{s}} d_{i j t} y^{j} y^{j} y^{t} \\
+\frac{1}{3} \frac{1}{x_{0}} x^{j} y_{j} d^{k l m} y_{k} y_{l} y_{m}+d_{i j k} d^{i l m} y^{j} y^{k} y_{l} y_{m} \gtrless 0 .
\end{array}\right] \\
& =(\Delta(x))^{2} \Delta(y) \gtrless 0,
\end{aligned}
$$

implying that $\Upsilon_{x}(y)$ lies in the same $\operatorname{Aut}\left(\mathfrak{F}\left(J_{3}\right)\right)$-orbit as $y$.

The same holds for a generic element $r=a y+b \Upsilon_{x}(y) \in \mathfrak{F}_{y \perp x}(a, b \in \mathbb{R})$, which belongs to the same $\operatorname{Aut}(\mathfrak{F})$-orbit as $y$ : indeed it can be checked that (consistently with eq. (4.23) and eq. (4.24))

$$
\begin{aligned}
\Delta(r) & =-2\left(a^{2}+\frac{2 b^{2}}{3} x_{0} d_{a b c} x^{a} x^{b} x^{c}\right)^{2}\left[\begin{array}{l}
\frac{1}{2}\left(-3 \frac{d_{k l m} y^{k} x^{l} x^{m}}{d_{q r s} x^{q} x^{r} x^{s}} x^{j} y_{j}+y^{j} y_{j}\right)^{2}+x_{0} \frac{d_{k l m} y^{k} x^{l} x^{m}}{d_{q r s} x^{q} x^{r} x^{s}} d_{i j t} y^{j} y^{j} y^{t} \\
+\frac{1}{3} \frac{1}{x_{0}} x^{j} y_{j} d^{k l m} y_{k} y_{l} y_{m}+d_{i j k} d^{i l m} y^{j} y^{k} y_{l} y_{m} \gtrless 0 .
\end{array}\right] \\
& =\left(a^{2}+\Delta(x) b^{2}\right)^{2} \Delta(y) \gtrless 0 .
\end{aligned}
$$

The canonical Darboux symplectic frame. We recall that in the $4 D / 5 D$ special coordinates' symplectic frame a generic element $Q$ of the reduced FTS $\mathfrak{F}\left(J_{3}\right)$ splits as given by eq. (8.1), while the $2 N \times 2 N$ symplectic metric is given by (6.9). By a simple re-ordering of rows and columns (amounting to a relabelling of indices, one can switch to a canonical Darboux symplectic frame ${ }^{31}$ (in which the $4 D / 5 D$ covariance is still manifest), in which $x$ eq. (8.1) splits as follows:

$$
x=-\left(p^{0}, q_{0}, p^{1}, q_{1}, \ldots, p^{N-1}, q_{N-1}\right)^{T},
$$

and in which the symplectic metric eq. (6.9) acquires the following form

$$
\Omega=-\mathbf{1} \otimes \varepsilon
$$

where $\varepsilon$ is the $2 \times 2$ symplectic metric of the defining irrepr. 2 of $\operatorname{Sp}(2) \approx \operatorname{SL}(2)$ defined by eq. (6.21).

At a glance, in a physical (Maxwell-Einstein) framework eq. (8.102) suggests that the choice of the (canonical) Darboux symplectic frame defined by (8.102) (or, equivalently, by eq. (8.103 and eq. (6.21)), amounts to making manifest the splitting of the electricmagnetic fluxes of the Abelian 2-form field strengths, grouped, within the symplectic vector $Q$ eq. (8.102), into the KK vector's fluxes (magnetic $p^{0}$ and electric $q_{0}$ ), and into the fluxes (magnetic $p^{i}$ and electric $q_{i}, i=1, \ldots, N-1$ ) of each of the $N-1$ Abelian vectors with a

\footnotetext{
${ }^{31}$ For some applications of the canonical Darboux frame to supergravity, see e.g. [77-79], and refs. therein.
} 
$D=5$ origin. When specifying such a generic (supersymmetry-independent) interpretation for minimal $D=5$ supergravity dimensionally reduced down to $\mathcal{N}=2, D=4$ supergravity, $p^{0}$ and $q_{0}$ are the magnetic resp. electric charges of the $D=4$ graviphoton (the Abelian vector in the $\mathcal{N}=2$ gravity multiplet), whereas each of the $N-1$ pairs $\left(p^{i}, q_{i}\right)$ denote the magnetic resp. electric charges of the Abelian vector belonging to each of the $N-1$ vector supermultiplets coupled to the gravity one (these all have a $D=5$ origin, thereby comprising the $D=5$ graviphoton, as well).

Thus, the $(2 N-2)$-dim. space $\mathfrak{F}_{x}^{\perp},\{$,$\} -orthogonal to the 2-dim. Freudenthal plane$ $\mathfrak{F}_{x}$, gets decomposed into $N-12$-dim. subspaces, all mutually orthogonal with respect to the symplectic product $\{$,$\} defined by (8.103): each of them corresponds to the electric-$ magnetic flux degrees of freedom of a vector supermultiplet in the corresponding $\mathcal{N}=2$, $D=4$ supergravity, or, more generally, to the electric-magnetic fluxes of a $D=4$ Abelian vector fields with a five-dimensional origin.

\subsection{F-duality preserves the $\Delta>0$ Aut( $\mathfrak{F})$-orbits: the $S T U$ model}

At least for (non-degenerate) reduced FTS's, Aut(F) has a transitive action on elements with a given $\Delta<0$. Thus, the $\Delta<0$ locus corresponds to a one-parameter family of $\operatorname{Aut}(\mathfrak{F})$-orbits; ${ }^{32}$ consequently, Freudenthal duality trivially preserves the orbit structure for $\Delta<0$.

The story is more complicated for the $\Delta>0$ locus, which, again at least for (nondegenerate) reduced FTS's, generically (with the unique exception of the $T^{3}$ model) has two or more Aut $(\mathfrak{F})$-orbits. However, the existence of the Freudenthal rotations presented in section 5.1 ensures that $x$ and $\tilde{x}$ always lie in the same orbit. Here we explicitly present the non-trivial orbit structure of the $\Delta>0$ locus and its properties under Freudenthal duality for the $S T U$ model using only the (discrete) U-duality invariants characterising the orbits.

The $S T U$ model, introduced independently in [81, 82], provides an interesting subsector of string compactification to four dimensions. This model has a low energy limit which is described by $\mathcal{N}=2$ supergravity coupled to three vector multiplets interacting through the special Kähler manifold $[\mathrm{SL}(2, \mathbb{R}) / \mathrm{SO}(2)]^{3}$. (In the version of $[81]$, the discrete $\mathrm{SL}(2, \mathbb{Z})$ are replaced by a subgroup denoted $\left.\Gamma_{0}(2)\right)$. The three complex scalars are denoted by the letters $S, T$ and $U$, hence the name of the model $[82,83]$. The remarkable feature that distinguishes it from generic $\mathcal{N}=2$ supergravities coupled to vectors [84] is its $S-$ $T-U$ triality [82]. There are three different versions with two of the $\operatorname{SL}(2)$ s perturbative symmetries of the Lagrangian and the third a non-perturbative symmetry of the equations of motion. In a fourth version all three are non-perturbative $[82,83]$. All four are onshell equivalent. If there are in addition four hypermultiplets, the $S T U$ model is selfmirror. Even though the simplest reduced FTS exhibiting more than one $\Delta>0 \operatorname{Aut}(\mathfrak{F})$ orbit is given by $\mathfrak{F}(\mathbb{R} \oplus \mathbb{R})$, which corresponds to the $S T^{2}$ model of $\mathcal{N}=2, D=4$ supergravity [80], we will explicitly treat $\mathfrak{F}(\mathbb{R} \oplus \mathbb{R} \oplus \mathbb{R})$ corresponding to the slightly larger

\footnotetext{
${ }^{32}$ We will equivalently use Aut $(\mathfrak{F})$, electric-magnetic duality, or U-duality, even if the first terminology is mathematical, the second more pertaining to Maxwell gravity, and the third one deriving from string theory.
} 
STU model, because it can be considered as a genuine truncation of all (non-degenerate) reduced FTS (with the exception of the $T^{3}$ and $S T^{2}$ models, which are however particular "degenerations" of the $S T U$ model itself), thus covering all such cases.

As determined in [80] (see also the treatment in section F.1 of [75]), in the $S T U$ model there are two orbits with $\Delta>0$, one supersymmetric and one non-supersymmetric (the one with vanishing central charge at the horizon: $Z_{H}=0$ ), and their coset expressions are isomorphic (even if they are $\mathrm{SL}(2, \mathbb{R})^{\times 3}$-disjoint orbits):

$$
\mathcal{O}_{\Delta>0, B P S} \cong \frac{\mathrm{SL}(2, \mathbb{R})^{\times 3}}{\mathrm{U}(1)^{\times 2}} \cong \mathcal{O}_{\Delta>0, n o n-B P S, Z_{H}=0}
$$

Following the treatment of [73], one can consider a $D 0-D 4$ representative (also in the FTS representation [17]) of the orbits $\mathcal{O}_{\triangle>0, B P S}$ and $\mathcal{O}_{\Delta>0, n o n-B P S, Z_{H}=0}$ :

$$
\begin{aligned}
x_{D 0-D 4, \Delta>0} & =\left(0, p^{1}, p^{2}, p^{3}, q_{0}, 0,0,0\right)^{T}=\left(\begin{array}{cc}
-q_{0} & \left(p^{1}, p^{2}, p^{3}\right) \\
(0,0,0) & 0
\end{array}\right), \\
\Delta\left(x_{D 0-D 4, \Delta>0}\right) & =4 q_{0} p^{1} p^{2} p^{3}>0,
\end{aligned}
$$

and determine the further $\operatorname{SL}(2, \mathbb{R})^{\times 3}$-invariant constraints on the sign of $q_{0}^{0}, p^{1}, p^{2}$ and $p^{3}$ defining the orbits $\mathcal{O}_{\triangle>0, B P S}$ and $\mathcal{O}_{\triangle>0, n o n-B P S, Z_{H}=0}$ within the $D 0-D 4$ configuration (8.105)-(8.110) with $\Delta>0$ (all other sign combinations pertain to the unique non-BPS $Z_{H} \neq 0$ orbit $\left.\mathcal{O}_{\Delta<0}\right)$ :

- $\frac{1}{2}$-BPS conditions (triality invariant):

$$
\left(q_{0}, p^{1}, p^{2}, p^{3}\right)=(+,+,+,+) \text { or }(-,-,-,-) .
$$

- non-BPS $Z_{H}=0$ conditions (up to triality) :

$$
\left(q_{0}, p^{1}, p^{2}, p^{3}\right)=(+,+,-,-) \text { or }(-,-,+,+) .
$$

Analogously, we can consider (also in the FTS representation [17]) a $D 2-D 6$ representative of the orbits $\mathcal{O}_{\triangle>0, B P S}$ and $\mathcal{O}_{\triangle>0, \text { non }-B P S, Z_{H}=0}$ :

$$
\begin{aligned}
x_{D 2-D 6, \Delta>0} & =\left(p^{0}, 0,0,0,0, q_{1}, q_{2}, q_{3}\right)^{T}=\left(\begin{array}{cc}
0 & (0,0,0) \\
\left(q_{1}, q_{2}, q_{3}\right) & p^{0}
\end{array}\right), \\
\Delta\left(x_{D 2-D 6, \Delta>0}\right) & =-4 p^{0} q_{1} q_{2} q_{3}>0 .
\end{aligned}
$$

From the treatment of [80], we can write down the further $\operatorname{SL}(2, \mathbb{R})^{\times 3}$-invariant constraints on the sign of $p^{0}, q_{1}, q_{2}$ and $q_{3}$ defining the orbits $\mathcal{O}_{\Delta>0, B P S}$ and $\mathcal{O}_{\Delta>0, n o n-B P S, Z_{H}=0}$ within the $D 2-D 6$ configuration eqs. (8.109)-(8.110) with $\Delta>0$ (all other sign combinations pertain to the unique non-BPS $Z_{H} \neq 0$ orbit $\left.\mathcal{O}_{\Delta<0}\right)$ :

- $\frac{1}{2}$-BPS conditions (triality invariant):

$$
\left(q_{0}, p^{1}, p^{2}, p^{3}\right)=(+,-,-,-) \text { or }(-,+,+,+) .
$$


- non-BPS $Z_{H}=0$ conditions (up to triality):

$$
\left(q_{0}, p^{1}, p^{2}, p^{3}\right)=(+,-,+,+) \text { or }(-,+,-,-) .
$$

As computed in Example 1 of section V.A of [17] (cfr. (198)-(200) therein), the Freudenthal dual of the $D 0-D 4$ element eqs. (8.105)-(8.106) yields a particular element of the type $D 2-D 6$ type eqs. (8.109)-(8.110) (up to triality; $c(m, n)>0$ ):

$$
\begin{aligned}
x_{D 0-D 4, \Delta>0} & =\left(\begin{array}{cc}
-n & (n,-m,-m) \\
(0,0,0) & 0
\end{array}\right) \\
\stackrel{\sim}{\longrightarrow} x_{D 0-D 4, \Delta>0} & =c(m, n)\left(\begin{array}{cc}
0 & (0,0,0) \\
(m,-n,-n) & -m
\end{array}\right) .
\end{aligned}
$$

By using eq. (8.113), one can then compute that the action of Freudenthal duality on the $D 0-D 4$ element (8.105)-(8.106) maps the conditions eq. (8.107) and eq. (8.108) respectively into the conditions eq. (8.111) and eq. (8.112): by using the commutativity of $\operatorname{Aut}(\mathfrak{F}(\mathbb{R} \oplus \mathbb{R} \oplus \mathbb{R}))=\mathrm{SL}(2, \mathbb{R})^{\times 3}$ and Freudenthal duality $\sim$ [17], one can consequently conclude that the orbit stratification of the $\Delta>0$ locus of $\mathfrak{F}(\mathbb{R} \oplus \mathbb{R} \oplus \mathbb{R})$ is preserved under Freudenthal duality $\sim$.

\section{Linear realisations of general Freudenthal transformations}

As consequence of its definition, Freudenthal duality $\sim$ can only be consistently defined in the locus $\Delta \neq 0$ of the FTS $\mathfrak{F}$ itself. In general, the group Aut $(\mathfrak{F})$ has a non-transitive action over such a locus, which undergoes a, (at least) twofold stratification, into a (always unique) $\Delta<0$ orbit and into a $\Delta>0$ sub-locus, which may in turn further stratify into $\operatorname{Aut}(\mathfrak{F})$-orbits.

While F-duality is a non-linear operation, as discussed in [17] its action can be realised by finite "local/gauged" U-duality transformations $U: \mathfrak{F} \rightarrow \operatorname{Aut}(\mathfrak{F})$, namely, as we will understand throughout the following treatment, that depend on the element of $\mathfrak{F}$ they are applied onto.

More generally, Freudenthal duality, and so GFT, can be mimicked by finite transformations of at least three different kinds, the first two of which are not contained in the U-duality group:

1. Gauged anti-symplectic transformations, as we will discuss, within non-degenerate, reduced FTS's, for the $\Delta<0$ orbit in subsection 9.1 ;

2. Gauged $\operatorname{Sp}(2 N, \mathbb{R})$ transformations, where $\operatorname{dim}_{\mathbb{R}} \mathfrak{F}=2 N$. We will discuss these, within the $T^{3}$ model $(N=2$; cfr. (table 1$)$ ), for the $\Delta<0$ orbit in subsection 9.2 ;

3. Gauged Aut $(\mathfrak{F})$ transformations, as we will discuss, within the $T^{3}$ model, for the $\Delta<0$ and $\Delta>0$ orbit in subsections 9.3 and 9.4 , respectively. 


\subsection{Anti-symplectic realisation: $\Delta<0$}

At least within (non-degenerate) reduced FTS's, the quartic polynomial invariant $\Delta$ of $\operatorname{Aut}\left(\mathfrak{F}\left(J_{3}\right)\right) \approx \operatorname{Conf}\left(J_{3}\right)$ can be written as in eq. (8.4) (see section 8 for this and other expressions) (cfr. e.g. [44, 72, 73]; $(i=1, \ldots, N-1, \operatorname{dim} \mathfrak{F}=2 N))$. Let us consider a $D 0-D 6$ configuration, studied in subsection 8.2. As we can seen there (we refer to eqs. (8.52), (8.53), (8.58) and (8.59)), the action of the Freudenthal duality $\sim$ on $x_{D 0 D 6}$ eq. (8.52) can be represented by a (maximal-rank) $2 N \times 2 N$ matrix

$$
\begin{aligned}
& \widetilde{x}=-\epsilon \mathbf{O} x, \\
& \mathbf{O}=\left(\begin{array}{llll}
1 & & & \\
& \mathbf{A} & & \\
& & -1 & \\
& & & \mathbf{B}
\end{array}\right),
\end{aligned}
$$

where $\mathbf{A}, \mathbf{B} \in \mathrm{GL}(N-1, \mathbb{R})$. The action of a general Freudenthal transformations is then

$$
x_{F}=a x+b \tilde{x}=(\mathbf{1}-\epsilon \mathbf{O}) x .
$$

The transformation $\mathbf{O} \in \mathrm{GL}(2 N, \mathbb{R})$ is inherently not unique. Also, apart from the "干" branching in (9.1), the whole realisation of Freudenthal duality does not depend on $p^{0}$ nor on $q_{0}$, and so it can be (loosely) considered an "ungauged" transformation in $\mathfrak{F}$. In particular, $\mathbf{O}$ is anti-symplectic, namely

$$
\mathbf{O}^{T} \Omega \mathbf{O}=-\Omega \Leftrightarrow \mathbf{A}^{T} \mathbf{B}=-\mathbf{1}
$$

where $\mathbf{1}$ is the identity matrix in $N-1$ dimensions. Note, however, that $\mathbf{O}$ is never symplectic (i.e., it always holds that $\mathbf{O}^{T} \Omega \mathbf{O} \neq \Omega \Leftrightarrow \mathbf{O} \notin S p(2 N, \mathbb{R})$ ).

Let us consider a particularly simple anti-symplectic case, for which $\mathbf{A}=-\mathbf{1} \Rightarrow \mathbf{B}=\mathbf{1}$ :

$$
\mathbf{O}^{\prime}=\left(\begin{array}{cccc}
1 & & & \\
& -\mathbf{1} & & \\
& & -1 & \\
& & & \mathbf{1}
\end{array}\right) \text {. }
$$

At least in all reduced FTS's $\mathfrak{F}$ 's based on simple and semi-simple rank-3 Jordan algebras (see section 2 and table 1 therein), it can be proved (cfr. appendix D of [85]) that $\mathbf{O}^{\prime}$ eq. (9.5) realizes an outer automorphism of $\operatorname{Aut}\left(\mathfrak{F}\left(J_{3}\right)\right)$, namely that

$$
\begin{aligned}
\mathbf{O}^{-1} \widehat{\mathbf{R}}_{\mathfrak{F}}(g) \mathbf{O} & \subset \widehat{\mathbf{R}}_{\mathfrak{F}}(g), \\
\mathbf{O} & \in \frac{\operatorname{Aut}\left(\operatorname{Aut}\left(\mathfrak{F}\left(J_{3}\right)\right)\right)}{\operatorname{Inn}\left(\operatorname{Aut}\left(\mathfrak{F}\left(J_{3}\right)\right)\right)} \equiv \operatorname{Out}\left(\operatorname{Aut}\left(\mathfrak{F}\left(J_{3}\right)\right)\right),
\end{aligned}
$$

where $\widehat{\mathbf{R}}_{\mathfrak{F}}(g)$ denotes the $2 N \times 2 N$ matrix representation of the element $g$ of $\operatorname{Aut}\left(\mathfrak{F}\left(J_{3}\right)\right)$ acting on $\mathfrak{F}$ itself, and Aut, Inn and Out respectively denote the automorphism group, and its inner resp. outer components. Note that $\mathbf{O}^{\prime}$ is an involution:

$$
\mathrm{O}^{\prime 2}=1
$$


where here 1 denotes the the identity matrix in $2 N$ dimensions. However, from eqs. (6.7)-(8.59) it follows that the correct iteration of the Freudenthal duality on the $D 0-D 6$ configuration $x$ eq. (8.52) is provided by the application of $\mp \mathbf{O}^{\prime}$ and then necessarily of $\pm \mathbf{O}^{\prime}$, thus yielding to $-\mathbf{O}^{\prime 2}=-\mathbf{1}$ acting on $x$, and correctly implying

$$
\widetilde{\widetilde{x}}=-x \text {. }
$$

The anti-simplecticity of $\mathbf{O}^{\prime}$ eq. (9.5) implies that it does not preserve the symplectic structure of $\mathfrak{F}$ (as neither Freudenthal duality does, as well [17]). This is consistent with the fact that $\mathbf{O}^{\prime}$ realizes an outer automorphism of the electric-magnetic U-duality group Aut $\left(\mathfrak{F}\left(J_{3}\right)\right)$, which in turn is generally realized in a symplectic way $[23,86,87]$ :

$$
\widehat{\mathbf{R}}_{\mathfrak{F}}(g)^{T} \Omega \widehat{\mathbf{R}}_{\mathfrak{F}}(g)=\Omega, \forall g \in \operatorname{Aut}\left(\mathfrak{F}\left(J_{3}\right)\right) .
$$

\subsubsection{Anti-symplectic symmetries and parity transformations}

As observed in [85], at least for all automorphism groups of reduced FTS's over simple or semi-simple rank-3 Jordan algebras it holds that (see e.g. [88])

$$
\operatorname{Out}\left(\operatorname{Aut}\left(\mathfrak{F}\left(J_{3}\right)\right)\right) \subseteq \mathbb{Z}_{2} \text {. }
$$

Thus, all non-trivial elements of $\operatorname{Out}\left(\operatorname{Aut}\left(\mathfrak{F}\left(J_{3}\right)\right)\right)$ are implemented by anti-symplectic transformations. In [89] (also cfr. [90]), it was discussed that the global symmetry of the resulting Maxwell-Einstein (super)gravity contains the factor $\mathbb{Z}_{2}$, which can be offset by a spatial parity $\mathbf{P}$ transformation. In particular, from eq. (2.118) of [89], it follows that the global symmetry group $G$ of the resulting Maxwell-Einstein (super)gravity theory is given by

$$
G=G_{0} \times \mathbb{Z}_{2}=\left\{G_{0}, G_{0} \cdot \mathbf{p}\right\},
$$

where $G_{0}$ is the identity-connected, proper electric-magnetic $\left(U\right.$-)duality, Aut $\left(\mathfrak{F}\left(J_{3}\right)\right)$-part of $G$, whereas $\mathbf{p}$ corresponds to an element of $G$ implemented by an anti-symplectic transformation.

Interestingly, the above results relate a realisation (not the unique one, though! — see subsections 9.2 and 9.3) of the Freudenthal duality on the well-defined representative $D 0-$ $D 6$ of the unique, non-BPS (non-supersymmetric) rank- $4 \Delta<0$ orbit in $\mathfrak{F}$, to spatial parity transformations in the corresponding theory; in fact, anti-symplectic transformations, such as $\mathbf{O}^{\prime}$ eq. (9.5) are symmetries of the theory, provided that they are combined with spatial $(3 D)$ parity $\mathbf{P}$.

\subsection{Gauged symplectic realisation: $\Delta<0$}

It is also possible to find a (non-unique) symplectic transformation realizing the Freudenthal duality transformation eq. (6.7) on the $D 0-D 6$ representative of the $\Delta<0$ Aut (F)-orbit of $\mathfrak{F}$. However, this will necessarily be "gauged" in $\mathfrak{F}$, namely it will depend on the element of $F$ it acts upon (i.e., in this case, on the $D 0-D 6$ element eq. (8.52)). 
Let us exemplify this in the $T^{3}$ model $(N=2$, as from table 1$)$; we look for a finite $\operatorname{Sp}(4, \mathbb{R})$ transformation (realized as a $4 \times 4$ matrix $\mathcal{C}$ ) such that

$$
\mathcal{C}\left(\begin{array}{c}
p^{0} \\
0 \\
q_{0} \\
0
\end{array}\right)=\operatorname{sgn}\left(p^{0} q_{0}\right)\left(\begin{array}{c}
-p^{0} \\
0 \\
q_{0} \\
0
\end{array}\right)
$$

Defining $z \equiv q_{0} / p^{0}$, long but straightforward algebra yields to the following expression (for $\left.\operatorname{sgn}\left(p^{0} q_{0}\right)=\operatorname{sgn}(z)= \pm 1\right)$ :

$$
\mathcal{C}_{ \pm}(z ; a, b, c, d, e, f, g)=\left(\begin{array}{cccc} 
\pm\left(1+\frac{a}{z}\right) & \mp\left(b c \mp \frac{d e}{z}\right) & \frac{2}{z}+\frac{a}{z^{2}} & \mp\left(b f \mp \frac{d g}{z}\right) \\
\pm b z & e & b & g \\
a & -(b c z \mp d e) & \pm\left(1+\frac{a}{z}\right) & -(b f z \mp d g) \\
d & c & \pm \frac{d}{z} & f
\end{array}\right)_{e f-g c=1}
$$

$\mathcal{C}_{ \pm}$eq. (9.14) depends on $z$, and it is thus "gauged" in $\mathfrak{F}$. Moreover, it depends on 7 real parameters, with a constraint $(e f-g c=1)$ : therefore, it realizes a particular, $(D 0-D 6)$ dependent finite transformation of $\operatorname{Sp}(4, \mathbb{R})$, which mimicks the action of Freudenthal duality over the $D 0-D 6$ element eq. (8.52).

\subsection{Gauged Aut $(\mathfrak{F})$ realisation: $\Delta<0$}

In the $\Delta<0$ locus of $\mathfrak{F}$ (on which the action of $\operatorname{Aut}(\mathfrak{F}$ ) is always transitive, thus defining a unique orbit $\mathcal{O}_{\Delta<0}$ of $\mathfrak{F}$ ), we deal with the issue of mimicking the action of Freudenthal duality by an Aut $(\mathfrak{F})$ transformation (which will generally be local in $\mathfrak{F}$ ), and consider the following (commutative) diagram:

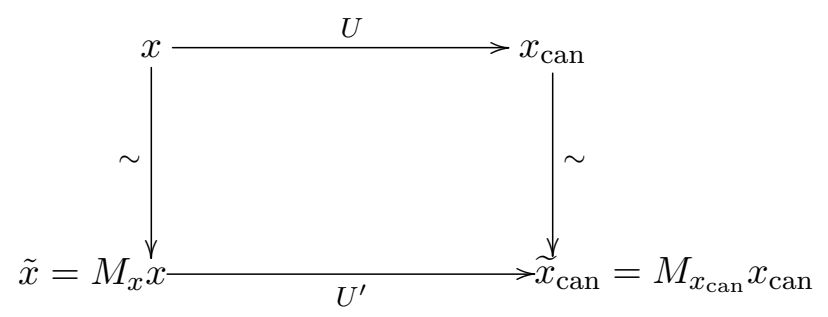

where $x, x_{\text {can }} \in \mathcal{O}_{\Delta<0}$. Here, $x_{\text {can }}$ denotes a convenient "canonical" representative that can be defined in a uniform manner for all relevant FTS as in [45]. The corresponding $\operatorname{Aut}(\mathfrak{F})$ transformations taking $x$ and $\tilde{x}$ to $x_{\text {can }}$ and $\tilde{x}_{\text {can }}$, respectively, are denoted $U$ and $U^{\prime}$. Similarly, $M_{x}$ and $M_{x_{\text {can }}}$ are the gauged Aut (F) transformations that send $x$ and $x_{\text {can }}$ to $\tilde{x}$ and $\tilde{x}_{\text {can }}$, respectively. Since the square commutes we free to pick a convenient canonical representative.

Generally, at least for (non-degenerate) reduced FTS's, the homogeneous space $\mathcal{O}_{\Delta<0}$ can be written as

$$
\mathcal{O}_{\Delta<0}=\frac{\operatorname{Conf}\left(J_{3}\right)}{\operatorname{Str}_{0}\left(J_{3}\right)}
$$


where we recall that $\operatorname{Aut}\left(\mathfrak{F}\left(J_{3}\right)\right) \simeq \operatorname{Conf}\left(J_{3}\right)$, Conf $\left(J_{3}\right)$ and $\operatorname{Str}_{0}\left(J_{3}\right)$ respectively denote the conformal and reduced structure groups of the cubic Jordan algebra $J_{3}$.

For simplicity's sake, let us assume $\operatorname{Str}_{0}\left(J_{3}\right)=I d$ (namely, there is no continuous nor discrete stabiliser for $\mathcal{O}_{\Delta<0}$, which thus is a group manifold: $\mathcal{O}_{\Delta<0} \cong$ Aut $(\mathfrak{F})$ ). Actually, this only holds for the $T^{3}$ model of $\mathcal{N}=2, D=4$ supergravity, associated to the simplest example of (non-degenerate) reduced FTS [75, 80]. Let us also choose a convenient representative of $\mathcal{O}_{\Delta<0}$. An obvious choice is given by the $D 0--D 6$ configuration eq. (8.52), $x_{\text {can }}=x_{D 0 D 6}$, which makes the $\operatorname{Str}_{0}\left(J_{3}\right)$ stabiliser of $\mathcal{O}_{\Delta<0}$ manifest for all reduced FTS.

The assumption $\operatorname{Str}_{0}\left(J_{3}\right)=I d$ implies $U^{\prime}=U$. From eq. (9.15) we have

$$
\begin{aligned}
\widetilde{x}_{D 0 D 6} & =\widetilde{U x} \\
& =U^{\prime} M_{x} x=U^{\prime} \widetilde{x}=\widetilde{U^{\prime} x} ;
\end{aligned}
$$

where the last step of the second line follows from the fact that Aut $(\mathfrak{F})$ and Freudenthal duality commute [17]. Thus, applying Freudenthal duality to (9.17) one obtains

$$
\left.U x=U^{\prime} x \Rightarrow x=U^{\prime-1} U x \Leftrightarrow \begin{array}{c}
U^{\prime-1} U \\
U^{-1} U^{\prime}
\end{array}\right\} \in \operatorname{Stab}(x)=I d \Leftrightarrow U=U^{\prime}
$$

Consequently, any reasoning involving the diagram eq. (9.15) is independent of the choice of $x_{\text {can }}$; indeed, the Aut $\left(\mathfrak{F}\left(J_{3}\right)\right)$ transformation connecting any two elements of $\mathcal{O}_{\Delta<0}=$ $\operatorname{Aut}\left(\mathfrak{F}\left(J_{3}\right)\right)$ (say $x_{\text {can }}$ and $x_{\text {can }}^{\prime}$ ) will be unique, since Aut $\left(\mathfrak{F}\left(J_{3}\right)\right)$ is free on the orbit $\mathcal{O}_{\Delta<0}$ by assumption.

In order to determine the gauged Aut $\left(\mathfrak{F}\left(J_{3}\right)\right)$ transformation $M_{x_{D 0 D 6}}$ mimicking Freudenthal duality acting on $x_{\text {can }}=x_{D 0 D 6}$, i.e. such that $\widetilde{x}_{D 0 D 6}=M_{x_{D 0 D 6} x_{D 0 D 6}}$ (cfr. eq. (9.15)), we will use the " $T^{3}$ degeneration" of the quantum information symplectic frame of the $S T U$ model (see e.g. [36, 75, 80, 91, 92]). By recalling that the usual parametrization of an element $x \in \mathfrak{F}\left(J_{3}\right)$ as a formal $2 \times 2$ matrix reads [17]

$$
x=\left(\begin{array}{cc}
\alpha & A \\
B & \beta
\end{array}\right), \alpha, \beta \in \mathbb{R}, A \in J_{3}, B \in \overline{J_{3}},
$$

the relation between the quantum information symplectic frame $x=a$, with $a_{a b c}=a_{(a b c)}$ $(a, b, c=0,1)$, the $4 D / 5 D$ special coordinates' symplectic frame $x=\left(p^{0}, p^{1}, q_{0}, q_{1}\right)^{T}$ and the FTS parametrization eq. (9.19) for the $T^{3}$ model $\left(J_{3}=\mathbb{R}\right)$ reads as follows:

$$
\begin{array}{rllll}
\text { QIT frame: } & a_{000} & a_{111} & a_{001} & a_{110}, \\
4 D / 5 D \text { frame: } & p^{0} & q_{0} & -p^{1} & q_{1} / 3, \\
\text { FTS frame: } & \beta & -\alpha & -A & B / 3,
\end{array}
$$

which is consistent with the " $T^{3}$ degeneration" of (175) and (183), table VII of [17].

Thus, we are searching for a $2 \times 2$ matrix $\mathbf{M}$ such that

$$
a_{(a b c) D 0 D 6}=\mathbf{M}_{a}^{a^{\prime}} \mathbf{M}_{b}^{b^{\prime}} \mathbf{M}_{c}^{c^{\prime}} a_{\left(a^{\prime} b^{\prime} c^{\prime}\right) D 0 D 6} \equiv M_{x_{D 0 D 6}}\left(\begin{array}{cc}
-q_{0} & 0 \\
0 & p^{0}
\end{array}\right) .
$$


Straightforward algebra yields the unique solution:

$$
\mathbf{M}(z)=\operatorname{sgn}(z)\left(\begin{array}{cc}
0 & -z^{-1 / 3} \\
z^{1 / 3} & 0
\end{array}\right), \quad z=q_{0} / p^{0} .
$$

Note that if and only if $z= \pm 1 \Leftrightarrow p^{0}= \pm q_{0}$, the matrix $\mathbf{M}(z= \pm 1)$ eq. (9.22) (and thus, through (9.21), $\left.M_{x_{D 0 D 6}}\right)$ belongs to $\mathrm{SL}(2, \mathbb{Z})$ :

$$
\mathbf{M}(z= \pm 1)= \pm\left(\begin{array}{cc}
0 & -1 \\
1 & 0
\end{array}\right)= \pm \varepsilon \in \operatorname{SL}(2, \mathbb{Z})
$$

where $\varepsilon$ is nothing but the symplectic $2 \times 2$ metric $\Omega_{2 \times 2}$ :

$$
\varepsilon \equiv\left(\begin{array}{cc}
0 & -1 \\
1 & 0
\end{array}\right) \equiv \Omega_{2 \times 2}
$$

Note that for $p^{0}, q_{0}= \pm 1$ the black hole is projective, cfr. the treatment in the quantized charge regime presented in [17]). The integral automorphism group $\operatorname{Aut}\left(\mathfrak{F}_{\mathbb{Z}}\right)$ acts transitively on projective charge configurations.

With the generalisation to arbitrary reduced FTS in mind, it is useful to reexpress $M_{x_{D 0 D 6}}(z)$, as defined by eq. (9.21), through the elementary Aut $(\mathfrak{F})$ transformations defined in $[14,93]$ for generic reduced FTS

$$
M_{x_{D 0 D 6}}(z)=\psi\left(-|z|^{-1 / 3}\right) \circ \varphi\left(|z|^{1 / 3}\right) \circ \psi\left(-|z|^{-1 / 3}\right),
$$

where

$$
\begin{aligned}
& \varphi(C):\left(\begin{array}{ll}
\alpha & A \\
B & \beta
\end{array}\right) \mapsto\left(\begin{array}{c}
\alpha+(B, C)+\left(A, C^{\sharp}\right)+\beta N(C) A+\beta C \\
B+A \times C+\beta C^{\sharp} \\
\beta
\end{array}\right) ; \\
& \psi(D):\left(\begin{array}{ll}
\alpha & A \\
B & \beta
\end{array}\right) \mapsto\left(\begin{array}{cc}
\alpha & A+B \times D+\alpha D^{\sharp} \\
B+\alpha D & \beta+(A, D)+\left(B, D^{\sharp}\right)+\alpha N(D)
\end{array}\right) ; \\
& T(\tau):\left(\begin{array}{ll}
\alpha & A \\
B & \beta
\end{array}\right) \mapsto\left(\begin{array}{cc}
\lambda^{-1} \alpha & \tau A \\
{ }^{t} \tau^{-1} B & \lambda \beta
\end{array}\right) ;
\end{aligned}
$$

where $C, D \in J_{3}$ and $\tau \in \operatorname{Str}\left(J_{3}\right)$ s.t. $N(\tau A)=\lambda N(A)$. In this form it is straightforward to generalise to arbitrary cubic Jordan algebra as follows. Consider the F-dual pair given by

$$
w=\left(\begin{array}{cc}
\alpha & 0 \\
0 & \beta
\end{array}\right), \quad \tilde{w}=\operatorname{sgn}(\alpha \beta)\left(\begin{array}{cc}
\alpha & 0 \\
0 & -\beta
\end{array}\right) .
$$

For base point $E$ and $c, d \in \mathbb{R}$ we find

$$
w^{\prime}=\psi(d E) \circ \varphi(c E) \circ \psi(d E)(w)
$$

is given by

$$
w^{\prime}=\left(\begin{array}{cc}
(c d+1)^{3} \alpha+c^{3} \beta & (c d+1)\left(\alpha d^{2}(c d+2)^{2}+\beta c(c d+1)\right) E \\
(c d+1)\left(\beta c^{2}+\alpha d\left(c^{2} d^{2}+3 c d+2\right)\right) E & d^{3}(c d+2)^{3} \alpha+(c d+1)^{3} \beta
\end{array}\right) .
$$


Hence, on setting

$$
d=-\operatorname{sgn}(\alpha \beta)(\beta / \alpha)^{1 / 3}=-|\beta / \alpha|^{1 / 3}
$$

and

$$
c=\operatorname{sgn}(\alpha \beta)(\alpha / \beta)^{1 / 3}=|\beta / \alpha|^{1 / 3}
$$

we obtain

$$
w^{\prime}=\operatorname{sgn}(\alpha \beta)\left(\begin{array}{cc}
\alpha & 0 \\
0 & -\beta
\end{array}\right)=\tilde{w}
$$

as required. Using eq. (9.15) this gives an explicit realisation of a U-duality relating any F-dual pair $x, \tilde{x}$ with $\Delta(x)<0$. Explicitly, setting $x_{\text {can }}=w$ using $U$ and then applying $M_{w}=\psi(d E) \circ \varphi(c E) \circ \psi(d E)$ we have

$$
\tilde{x}=U^{\prime-1} M_{w} U x .
$$

Note, since any $x$ in a $\Delta(x)<0$ orbit has stabiliser $\operatorname{Str}_{0}\left(J_{3}\right)$ the U-duality transformations are generically non-unique,

$$
\tilde{x}=S_{\tilde{x}} U^{\prime-1} S_{\tilde{w}} M_{w} S_{w} U S_{x} x
$$

for arbitrary $S_{y} \in \operatorname{Stab}(y) \subset \operatorname{Aut}(\mathfrak{F})$.

It is also straightforward to define a $\hat{w} \in \mathfrak{F}$ such that

$$
\exp \left[\frac{\pi}{2} \Upsilon_{\hat{w}}\right] w=\tilde{w}
$$

where $\hat{w}$ is determined by $w$. Recall, for $x=(\alpha, \beta, A, B), y=(\gamma, \delta, C, D)$, the Freudenthal product

$$
\wedge: \mathfrak{F} \otimes \mathfrak{F} \rightarrow \operatorname{Hom}(\mathfrak{F})
$$

is defined by,

$$
x \wedge y \equiv \Phi_{(\phi, X, Y, \nu)}, \quad \text { where }\left\{\begin{array}{l}
\phi=-\frac{1}{2}(A \vee D+C \vee B), \\
X=-\frac{1}{4}(B \times D-\alpha C-\gamma A), \\
Y=\frac{1}{4}(A \times C-\beta D-\delta B), \\
\nu=\frac{1}{8}(\operatorname{Tr}(A, D)+\operatorname{Tr}(C, B)-3(\alpha \delta+\gamma \beta)),
\end{array}\right.
$$

and $A \vee B \in \mathfrak{S t r} J$ is defined by

$$
(A \vee B) C \equiv \frac{1}{2} \operatorname{Tr}(B, C) A+\frac{1}{6} \operatorname{Tr}(A, B) C-\frac{1}{2} B \times(A \times C) .
$$

The action of $\Phi: \mathfrak{F} \rightarrow \mathfrak{F}$ is given by

$$
\Phi_{(\phi, X, Y, \nu)}\left(\begin{array}{ll}
\alpha & A \\
B & \beta
\end{array}\right)=\left(\begin{array}{cc}
\alpha \nu+(X, B) & \phi A-\frac{1}{3} \nu A+Y \times B+\beta X \\
-{ }^{t} \phi B+\frac{1}{3} \nu B+X \times A+\alpha Y & -\beta \nu+(Y, A)
\end{array}\right) .
$$

The set of all such homomorphisms yields the automorphism Lie algebra,

$$
\mathfrak{A} \mathfrak{u t}(\mathfrak{F})=\left\{\Phi(\phi, X, Y, \nu) \in \operatorname{Hom}_{\mathbb{R}}(\mathfrak{F}) \mid \phi \in \mathfrak{S t r}_{0}(\mathfrak{J}), X, Y \in \mathfrak{J}, \nu \in \mathbb{R}\right\},
$$


where the Lie bracket

$$
\left[\Phi\left(\phi_{1}, X_{1}, Y_{1}, \nu_{1}\right), \Phi\left(\phi_{2}, X_{2}, Y_{2}, \nu_{2}\right)\right]=\Phi(\phi, X, Y, \nu)
$$

is given by

$$
\begin{aligned}
\phi & =\left[\phi_{1}, \phi_{2}\right]+2\left(X_{1} \vee Y_{2}-X_{2} \vee Y_{1}\right), \\
X & =\left(\phi_{1}+\frac{2}{3} \nu_{1}\right) X_{2}-\left(\phi_{2}+\frac{2}{3} \nu_{2}\right) X_{1}, \\
Y & =\left({ }^{t} \phi_{2}+\frac{2}{3} \nu_{2}\right) Y_{1}-\left({ }^{t} \phi_{1}+\frac{2}{3} \nu_{1}\right) Y_{2}, \\
\nu & =\operatorname{Tr}\left(X_{1}, Y_{2}\right)-\operatorname{Tr}\left(Y_{1}, X_{2}\right) .
\end{aligned}
$$

Noting that

$$
\varphi(C)=\exp [\Phi(0, C, 0,0)], \quad \psi(D)=\exp [\Phi(0,0, D, 0)]
$$

and, from eq. (9.38), that

$$
e \equiv \Phi(0, E, 0,0), \quad f \equiv \Phi(0,0, E, 0), \quad h \equiv \Phi(0,0,0,3),
$$

generates an $\mathfrak{s l}(2, \mathbb{R})$ subalgebra,

$$
[e, f]=h, \quad[h, e]=2 e, \quad[h, f]=-2 f,
$$

we find

$$
\psi(d E) \circ \varphi(-E / d) \circ \psi(d E)=\exp \left[\frac{\pi}{2} \Phi(0,-E / d, d E, 0)\right],
$$

which on setting $d=-|\beta / \alpha|^{1 / 3}$ gives the F-dual transformation eq. (9.30).

Using

$$
4 x \wedge x(y)=3 T(x, x, y)+\{x, y\} x
$$

and for $\hat{w}=2(0,0, \sqrt{d} E, E / \sqrt{d})$

$$
\hat{w} \wedge w=\Phi(0,-1 / d E, d E, 0)
$$

we have

$$
\exp \left[\frac{\pi}{2} \Upsilon_{\hat{w}}\right] w=\tilde{w}
$$

\subsection{Gauged Aut $(\mathfrak{F})$ realisation: $\Delta>0$}

As for the $\Delta(x)<0$ case treated above, when $\Delta(x)>0$ the action of Freudenthal duality on $x$ can generally be realised by linear gauged $\operatorname{Sp}(2 N, \mathbb{R})$ (recalling that $\operatorname{dim}_{\mathbb{R}} \mathfrak{F}=2 N$ ) or Aut $(\mathfrak{F})$ transformations. For simplicity's sake, we confine ourselves here to the study of the gauged $\operatorname{Aut}(\mathfrak{F})$ transformations. ${ }^{33}$

\footnotetext{
${ }^{33}$ At least in (non-degenerate) reduced FTS's, a similar treatment along the non-BPS $Z_{H}=0$ orbit $(\Delta>0)$ can be given. Within (non-degenerate) reduced FTS's, the smallest model exhibiting a non-BPS $\Delta>0$ orbit is the $S T^{2}$ model [80].
} 
Concerning the $\Delta>0$ locus of $\mathfrak{F}$, it is generally stratified in two or more orbits under the non-transitive action of $\operatorname{Aut}(\mathfrak{F})$. In the following treatment, we will consider the particularly simple case of the $T^{3}$ model, in which such a stratification does not take place, and thus the $\Delta>0$ locus of $\mathfrak{F}(\mathbb{R})$ corresponds to a unique $\operatorname{Aut}(\mathfrak{F})=\operatorname{SL}(2, \mathbb{R})$ supersymmetric $(1 / 2$-BPS) orbit $[75,80]$ :

$$
\mathfrak{F}(\mathbb{R})_{\Delta>0}=\mathcal{O}_{\Delta>0} \cong \mathrm{SL}(2, \mathbb{R}) / \mathbb{Z}_{3} .
$$

The non-trivial, discrete, stabiliser of the $\mathcal{O}_{\Delta>0}$ orbits for the $T^{3}$ model is up to conjugation given by the $\mathbb{Z}_{3} \subset \mathrm{SO}(2) \subset \mathrm{SL}(2, \mathbb{R})$, generated by [75]

$$
\hat{M} \equiv \frac{1}{2}\left(\begin{array}{cc}
-1 & \sqrt{3} \\
-\sqrt{3} & -1
\end{array}\right), \quad \operatorname{det} \hat{M}=1, \quad \hat{M}^{-1}=\hat{M}^{T} .
$$

As computed below, the Freudenthal dual of the $D 0-D 4$ representatives of the orbits $\mathcal{O}_{\Delta>0}$ are given by $D 2-D 6$ elements

$$
\widetilde{x}_{D 0 D 4}=\frac{1}{\sqrt{q_{0} p^{3}}}\left(-p^{3}, 0,0,3 q_{0} p^{2}\right) .
$$

In order to determine the gauged Aut $\left(\mathfrak{F}\left(J_{3}\right)\right)$ transformation $M_{x_{D 0 D}}$ mimicking, along $\mathcal{O}_{\Delta>0}$, Freudenthal duality acting on $x_{\text {can }}=x_{D 0 D 4}$, i.e. such that $\widetilde{x}_{D 0 D 4}=M_{x_{D 0 D 4}} x_{D 0 D 6}$ (cfr. eq. (9.15)), we will again use the " $T^{3}$ degeneration" of the quantum information symplectic frame of the $S T U$ model, namely we search for a $2 \times 2$ matrix IM such that

$$
\widetilde{a}_{(a b c) D 0 D 4}=\mathbb{M}_{a}^{a^{\prime}} \mathbb{M}_{b}^{b^{\prime}} \mathbb{M}_{c}^{c^{\prime}} a_{\left(a^{\prime} b^{\prime} c^{\prime}\right) D 0 D 4} \equiv M_{x_{D 0 D 4}} x_{D 0 D 4}
$$

where $x_{D 0 D 4}$ in the FTS parametrization (following the conventions of [75]) reads

$$
x_{D 0 D 4}=\left(\begin{array}{cc}
-q_{0} & p \\
0 & 0
\end{array}\right) .
$$

Long but straightforward algebra yields the twofold solution $\left(y=q_{0} / p>0\right)$ :

$$
\mathrm{M}_{ \pm}(y)=\left(\begin{array}{cc} 
\pm \frac{\sqrt{3}}{2} & \frac{1}{2} y^{-1 / 2} \\
-\frac{1}{2} y^{1 / 2} & \pm \frac{\sqrt{3}}{2}
\end{array}\right)
$$

$M_{x_{D 0 D 4}}(y)$ (defined by eq. (9.49)) can be realized in terms of the Aut $(\mathfrak{F}(\mathbb{R}))$ transformations eq. (9.26) as follows:

$$
M_{x_{D 0 D 4}}(y)=T\left( \pm \frac{\sqrt{3}}{2}\right) \circ \varphi\left(\mp \frac{\sqrt{3}}{4} y^{1 / 2}\right) \circ \psi\left( \pm \frac{1}{\sqrt{3}} y^{-1 / 2}\right) .
$$

It is interesting to note that for $y=1 \Leftrightarrow q_{0}=p$, the matrix $\mathbb{M}_{ \pm}(y=1)$ eq. (9.51) (and thus, through eq. (9.49), $\left.M_{x_{D 0 D 4}}\right)$ does not belong to $\mathrm{SL}(2, \mathbb{Z})$ :

$$
\mathrm{M}(y=1)=\left(\begin{array}{cc} 
\pm \frac{\sqrt{3}}{2} & \frac{1}{2} \\
-\frac{1}{2} & \pm \frac{\sqrt{3}}{2}
\end{array}\right) \notin \mathrm{SL}(2, \mathbb{Z}) .
$$


However, remarkably, only for $y=1$ another solution to eq. (9.49) can be found (the subscript "add" stands for additional; recall eq. (9.23)):

$$
\mathrm{M}_{\mathrm{add}}^{y=1}=\left(\begin{array}{cc}
0 & -1 \\
1 & 0
\end{array}\right)=\varepsilon=\mathrm{M}(z=1) \in \mathrm{SL}(2, \mathbb{Z}),
$$

with $\varepsilon$ defined by eq. (6.21). Due to the existence of the additional solution eq. (9.54), the integral (projective) case is obtained for $y=1$ (by further setting $p \in \mathbb{Z}$ ) (cfr. the treatment in the quantized charge regime, presented in [17]).

Recall, since we are assuming $\Delta(x)>0$, we can use the Freudenthal rotation in $\operatorname{Aut}(\mathfrak{F})$,

$$
\exp \left[\frac{\pi}{2} \bar{\Upsilon}_{x}\right] x=\tilde{x}
$$

Specialising to a generic ${ }^{34}$ element in the $D 0 D 4$ system

$$
w=\left(\begin{array}{cc}
\alpha & A \\
0 & 0
\end{array}\right), \quad \tilde{w}=\frac{1}{\sqrt{-\alpha N(A)}}\left(\begin{array}{cc}
0 & 0 \\
\alpha A^{\sharp} & N(A)
\end{array}\right),
$$

and using

$$
\bar{\Upsilon}_{x}=\frac{4}{3 \sqrt{\Delta(x)}} x \wedge x
$$

we have

$$
\bar{\Upsilon}_{w}=\frac{1}{3 \sqrt{-\alpha N(A)}} \Phi\left(0, \alpha A, A^{\sharp}, 0\right),
$$

so that

$$
\exp \left[\frac{\pi}{6 \sqrt{-\alpha N(A)}} \Phi\left(0, \alpha A, A^{\sharp}, 0\right)\right] w=\tilde{w} .
$$

Further restricting to the $T^{3}$ model and setting $\alpha=-q_{0}, A=p$ we find,

$$
\frac{\pi}{6 \sqrt{-\alpha N(A)}} \Phi\left(0, \alpha A, A^{\sharp}, 0\right)=\frac{\pi}{6} \Phi\left(0,-y^{1 / 2}, y^{-1 / 2}, 0\right)
$$

and

$$
\exp \left[\frac{\pi}{6} \Phi\left(0,-y^{1 / 2}, y^{-1 / 2}, 0\right)\right]=T\left(\frac{\sqrt{3}}{2}\right) \circ \varphi\left(-\frac{\sqrt{3}}{4} y^{1 / 2}\right) \circ \psi\left(\frac{1}{\sqrt{3}} y^{-1 / 2}\right)
$$

as given in eq. (9.52).

\footnotetext{
${ }^{34}$ Generically, this is actually a larger class of charge configurations, but in the special cases of the $T^{3}, S T^{2}, S T U$ models it is precisely the $D 0 D 4$ subsector. For $\mathcal{N}=8$, imposing $A$ is diagonal restricts to the $D 0 D 4$ subsector.
} 


\subsection{Non-trivial orbit stabilizers}

Here, generalizing the reasoning at the end of subsection 9.3, we want to reconsider the diagram eq. (9.15), and generalize the treatment to the case in which (regardless of the sign of $\Delta(x))$ the Aut $(\mathfrak{F})$-orbit to which $x \in \mathfrak{F}$ belongs is endowed with a non-trivial stabilizer $\mathcal{H}$, such that the corresponding homogeneous (generally non-symmetric) manifold can be written as

$$
x \in \mathcal{O}=\frac{A u t(\mathfrak{F})}{\mathcal{H}} \subset \mathfrak{F} .
$$

Let us deal with the issue of mimicking the action of Freudenthal duality by an Aut $(\mathfrak{F})$ finite transformation (which will generally be "gauged" in $\mathfrak{F}$ ), and consider again the (commutative) diagram eq. (9.15). In general ${ }^{35}\left(U, U^{\prime} \in \frac{A u t(\mathfrak{F})}{\mathcal{H}}\right)$, it holds that

$$
\begin{aligned}
& \widetilde{x}_{\text {can }}=\widetilde{U x} ; \\
& \widetilde{x}_{\text {can }}=U^{\prime} \widetilde{x}=\widetilde{U^{\prime} x},
\end{aligned}
$$

where in the last step of the second line we used the commutativity of Freudenthal duality and $\operatorname{Aut}(\mathfrak{F})$. Thus, eq. (9.63) implies

$$
x=U^{\prime-1} U x \quad \Leftrightarrow \quad U^{\prime-1} U=Z_{x} \in \operatorname{Stab}(x)
$$

On the other hand:

$$
\widetilde{x}=\widetilde{U^{-1} x_{\mathrm{can}}}, \quad \widetilde{x}=U^{\prime-1} \widetilde{x}_{\mathrm{can}}=\widetilde{U^{\prime-1} x_{\mathrm{can}}},
$$

where again in the last step we used the commutativity of Freudenthal duality and Aut ( $\mathfrak{F})$. Thus, (9.65) implies

$$
x_{\mathrm{can}}=U^{\prime} U^{-1} x_{\mathrm{can}} \Leftrightarrow U^{\prime} U^{-1}=Z_{x_{\mathrm{can}}} \in \operatorname{Stab}\left(x_{\mathrm{can}}\right) .
$$

Let us observe that

$$
\operatorname{Stab}(x)=\operatorname{Stab}(\widetilde{x})
$$

since for $Z_{x} \in \operatorname{Stab}(x)$ and $Z_{\widetilde{x}} \in \operatorname{Stab}(\widetilde{x})$ we have

$$
Z_{\widetilde{x}} \widetilde{x}=\widetilde{x}=\widetilde{Z_{x} x}=Z_{x} \widetilde{x}
$$

and vice versa, implying eq. (9.67).

By virtue of results eq. (9.64), eq. (9.66) and eq. (9.67), one can write

$$
U^{\prime} x=Z_{x_{\text {can }}} U Z_{x} x .
$$

Moreover, it is here worth commenting that, in presence of non-trivial $\mathcal{H}$, any reasoning involving the diagram eq. (9.15) is actually dependent from the actual choice of $x_{\text {can }}$; indeed, the Aut $\left(\mathfrak{F}\left(J_{3}\right)\right)$ transformation connecting any two elements of $\mathcal{O}$ (say $x_{\text {can }}$ and $x_{\text {can }}^{\prime}$ ) will not be unique, for the reasons highlighted above.

\footnotetext{
${ }^{35}$ If $U$ and $U^{\prime}$ are more general finite transformations, such as "gauged" symplectic (not belonging to $\operatorname{Aut}(\mathfrak{F}))$ or anti-symplectic transformations, things become more complicated, since, for instance, finite transformations of the pseudo-Riemannian non-compact non-symmetric coset $\operatorname{Sp}(2 n, \mathbb{R}) / \operatorname{Aut}(\mathfrak{F})$ do not

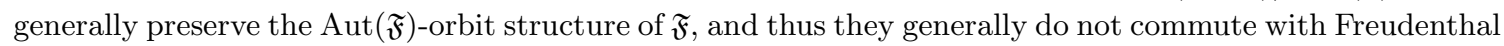
duality.
} 


\section{Summary, concluding and further remarks}

The purpose of this work has been to present, extend and clarify old and new results concerning general Freudenthal transformations, which generalise Freudenthal duality, while filling in necessary details not yet appearing in the mathematics or physics literature.

We begin with a detailed and self-contained treatment of FTS, groups of type $E_{7}$, F-duality and GFT in sections 2-5, laying the groundwork for the subsequent sections that apply the formalism to Einstein-Maxwell-Scalar (super)gravity theories.

In section 6 we study the entropy properties of $\mathcal{N}=2, D=4$ pure supergravity from the point of view of FTS formalism, where is it given by a non-reduced FTS with positive-definite quartic invariant. In this case, general Freudenthal transformations are nothing other than the familiar U(1) electromagnetic duality and Freudenthal duality is an anti-involutive duality transformation.

In section 7 we considered the axion-dilaton model, an $\mathcal{N}=2, d=4$ supergravity minimally coupled to one vector multiplet, which can be considered a consistent truncation of $\mathcal{N}=4$ supergravity. The mathematical structure is in this case a two dimensional FTS with negative-semi-definite quartic invariant. The corresponding extremal $\mathrm{BH}$ is non-BPS. A $S O_{0}(1,1)$ subgroup of the GFTs leave invariant the entropy. Freudenthal duality reduces in this case to a U-duality transformation. This model, as the pure supergravity studied before, cannot be uplifted to $D=5$, which corresponds directly to the fact that the associated FTS is not reduced.

In section 8 we proceeded to the analysis of $\mathcal{N}=2, D=4$ supergravities with a $D=5$ origin. The mathematical structure of these models is that of a FTS derived from a cubic Jordan Algebra. In first place we study the $T^{3}$ model, or in Freudenthal terminology a $\mathfrak{F}\left(J_{3}=\mathbb{R}\right)$ structure. This model is represented by the unique reduced FTS with $\operatorname{dim} \mathfrak{F}=4$ as vector space. The automorphism group is a four dimensional representation of $\operatorname{SL}(2, \mathbb{R})$ which can be decomposed into $\mathrm{SO}(1,1)$ representations, making the $D=5$ origin manifest. The full space is split into two two-dimensional planes orthogonal (with respect to the symplectic bilinear form), which can be identified respectively with purely $D 6-D 0$ or $D 4-D 2$ configurations. Other identifications are, however, possible, for example a $D 4-$ $D 0 / D 6-D 2$ decomposition. General Freudenthal transformations leave invariant the entropy for configurations chosen in each of the planes for every decomposition. Any $D 6-D 0$ configuration has a negative value for the quartic invariant, corresponding to extremal non-BPS BHs. Meanwhile, the D4-D2 configurations correspond to BHs with either positive (BPS or non-BPS) or negative (non-BPS) quartic invariant. Each nonBPS $D 6-D 0 \mathrm{BH}$ can be put in correspondence with a BPS $D 4-D 2$ one, with the same entropy. Mathematically this correspondence is performed by a $\Upsilon_{x}$ map, which exponentiates to an element of $\operatorname{Aut}(\mathfrak{F})$. It is also possible to consider an initial family of only $D 4-D 0$ configurations. This family includes both BPS and non-BPS BHs (respectively configurations with positive and negative quartic invariants). The application of general Freudenthal transformations to any member of this family generates a two-parameter subset of the full $D 6-D 4-D 0-D 2$ configuration space. The configurations with $D 6$ or $D 4$ charges alone are "small" $(\Delta=0) \mathrm{BH}$. They are related among themselves by general 
Freudenthal transformations forming a $D 6-D 4$ plane, which is $\Upsilon$-mapped to a $D 2-D 0$ plane. Similar results are obtained in general theories beyond $T^{3}$ containing an arbitrary number of charges, they are studied in full detail in subsection 8.2 and subsection 8.3.

General Freudenthal transformations, and in particular Freudenthal duality $\sim$, will preserve the orbit structure for $\Delta<0$. This is trivially true since, at least for (nondegenerate) reduced FTS, Aut(F) has a transitive action on elements with a given $\Delta<0$. The situation is more complicated for the locus of $\Delta>0$ configurations, for which, in general (with known exceptions) are stratified in two or more automorphism orbits. In subsection 8.4 we study this question and conclude that the orbit stratification of the $\Delta>0$ locus of $\mathfrak{F}(\mathbb{R} \oplus \mathbb{R} \oplus \mathbb{R})$ is preserved under Freudenthal duality $\sim$, and by extension by General Freudenthal transformations. This result generalises to all cases with (nondegenerate) reduced FTS, as they can either be invectively embedded in, or truncated to, the $S T U$ model.

In section 9 we show, in different examples, how the action of General Freudenthal transformations, and, in particular, Freudenthal duality can be mimicked/undone by finite U-duality $(\operatorname{Aut}(\mathfrak{F}))$, transformations which are gauged in that they depend on the element of $\mathfrak{F}$ they are applied onto. We restrict to two situations. First, for configurations within the $\Delta<0$ locus of $\mathfrak{F}$, the action of Aut $(\mathfrak{F})$ is always transitive on elements of a given $\Delta$, thus defining a unique one-parameter family of orbits $\{\mathcal{O}\}_{\Delta(x)<0}$ of $\mathfrak{F}$. The $\Delta>0$ locus of $\mathfrak{F}$ is generally stratified in two or more orbits for any fixed $\Delta>0$ under the non-transitive action of $\operatorname{Aut}(\mathfrak{F})$. However it is possible to find particular cases where this stratification does not take place. For example, the $T^{3}$ model has a unique orbit for all $\Delta>0$ under the $\mathrm{SL}(2, \mathbb{R})$ automorphism group. Hence, for the $T^{3}$ model all BH with $\Delta>0$ are $(1 / 2)-\mathrm{BPS}$. This example is studied in subsection 9.4.

The entropy of a linear superposition of configurations $x, y$ is given by

$$
\frac{S_{B H}^{2}(x+y)}{4 \pi}=|\Delta(x+y)|,
$$

which is not the sum of the entropies of the individual constituents

$$
\Delta(x+y)=\Delta(x)+\Delta(y)+3\left\{x^{\prime}, y\right\}+3\left\{x, y^{\prime}\right\}+\left\{y, \Upsilon_{x}(y)\right\}
$$

However for linear combinations of the form given by a General Freudenthal transformation, $x_{F}=a x+b \tilde{x}$, the entropy of the composite object is simply related to that one of $x$

$$
\frac{S_{B H}^{2}\left(x_{F}\right)}{4 \pi}=\left|a^{2}+\epsilon b^{2}\right| \frac{S_{B H}^{2}(x)}{4 \pi} .
$$

Thus there is a family of configurations for which the entropy is the same to the entropy of $x$, those with $a^{2}+\epsilon b^{2}= \pm 1$, with $\epsilon=\operatorname{sgn} \Delta(x)$.

We can use these results to show that it is possible to construct asymptotically "small" (zero entropy) interacting black holes from an initial non-trivial configuration. First, when $\Delta(x)>0(\epsilon=1)$, the elements defined by (see also eq. (B.36)) $x_{F \pm}=x \pm \tilde{x}$ are rank-4 element of $\mathfrak{F}_{x} \subset \mathfrak{F}$ (see eq. (B.37) and table 2). However, when $\Delta(x)<0(\epsilon=-1)$ it 
follows that $x_{F \pm}$ are null elements ${ }^{36}$

$$
\Delta\left(x_{F \pm}\right) \equiv \Delta(x \pm \tilde{x})=0 .
$$

with vanishing Bekenstein-Hawking entropy

$$
S_{B H}(x \pm \tilde{x})=0 .
$$

This suggests the existence of a class of "two-centered black hole solutions" where each centre is "large" non-BPS $(\Delta(x)=\Delta( \pm \tilde{x})<0)$, they are interacting since $\{\tilde{x}, x\} \neq 0$, yet asymptotically their Bekenstein-Hawking entropy vanishes, so the total system (before crossing a line of marginal stability) belongs to a small nilpotent ${ }^{37}$ orbit. The physical or geometric significance of such configurations remains unclear.

Alternatively, small BH solutions can constructed by the application of the properties of $\Upsilon_{x} \in \mathfrak{a} \mathfrak{u t}(\mathfrak{F})$ maps. The behaviour of $S_{B H}$ (or $\Delta$ ) on the $\mathfrak{F}$ or $\mathfrak{F}_{y \perp x}$ planes is similar (see subsection 4.1). The null elements of $\mathfrak{F}_{x}$ and any $\mathfrak{F}_{y \perp x}$ are "aligned". The locus of null entropy, is given by the same eq. (4.29) which it is independent of $y$. For $\Delta(x)<0, y \in \mathfrak{F}_{x}^{\perp}$, any element of the form

$$
z_{ \pm}=\sqrt{|\Delta(x)|} y \pm \Upsilon_{x}(y)
$$

is null, $\Delta\left(z_{ \pm}\right)=0$. This describes another class of two-centred black hole configurations, which are interacting since $\left\{y, \Upsilon_{x} y\right\} \neq 0$, yet asymptotically their Bekenstein-Hawking entropy vanishes

$$
S_{B H}\left(y \pm \bar{\Upsilon}_{x}(y)\right)=0 .
$$

Further work on such small BHs will be presented elsewhere.

The extension of these results to systems with quantized charges is challenging. In this case the requirement that the set of charge vectors $x_{f}=a x+b \tilde{x}$ belongs to the charge lattice is extremely restrictive. Let us recall that for the case of Freudenthal duality, demanding that $x, \tilde{x}$ are integers restrict us to a open subset of black holes where the entropy is necessarily an integer multiple of $\pi$. The complete characterisation of discrete U-duality invariants, which may or may not also be F- and GFT invariant, remains an open question and, hence, so does the F-dual invariance of higher order corrections to the entropy.

Let us just present a simple result. In the case of $\mathcal{N}=8, D=4$, the automorphism group is $E_{7(7)}(\mathbb{Z})$ and $\Delta(x)$ is quantised [48], $\Delta(x)=0_{[4]}$ or $\Delta(x)=1_{[4]}$, where $n_{[4]} \equiv n$ mod 4 . The requirement that both $x, \tilde{x}$ are integer restricts us to the subset of black holes for which $\pm \Delta$ is a perfect square among other conditions [48]. Let us explore what happens

${ }^{36}$ The elements $x_{F \pm}$ are actually rank-1 element of $\mathfrak{F}_{x} \subset \mathfrak{F}$. In this case (eq. (B.37), table 2)

$$
\begin{aligned}
\left(x_{F \pm}\right)^{\prime} \equiv(x \pm \tilde{x})^{\prime} & =0, \\
\Upsilon_{x_{F \pm}}(y) & =0, \forall y \in \mathfrak{F} .
\end{aligned}
$$

In particular it does not have a well defined $\tilde{\mathrm{F}}$-dual.

${ }^{37}$ In the $D=3$ language; cfr. e.g. the nilpotent orbits of $\mathfrak{s o}(4,4)$ acting on its adjoint irrep. 28 in [96]. 
for integral GFT. Noting that the F-dual scales linearly $(\tilde{n x})=n \tilde{x}, n \in \mathbb{Z}$, according to eq. (3.13), we find,

$$
\Delta(n x+m \tilde{x})=\left(n^{2}+\epsilon m^{2}\right)^{2} \Delta(x), \quad n, m \in \mathbb{Z} .
$$

From the structure of this expression it is obvious that the requirement the entropy being a perfect square is automatically preserved under a GFT.

Finally, we note that the charges of five dimensional stringy black holes may be described in the context of (cubic) Jordan algebras. The cubic norm defining the relevant algebra determines the BH entropy to lowest order. The Jordan dual (introduced in [48]) is related to the Freudenthal dual of the corresponding 4D model. The generalization of this $4 \mathrm{D} / 5 \mathrm{D}$ correspondence from a general Freudenthal transformation to the corresponding putative "General Jordan Transformations" (GJT), of which the Jordan dual is a particular case, will be treated elsewhere [94].

\section{Acknowledgments}

The work of LB is supported by a Schrödinger Fellowship. The work of MJD was supported in part by the STFC under rolling grant ST/P000762/1. The work of AM has been partially supported by a fellowship from Fundacion Seneca, CARM, Spain. AM would like to thank the hospitality of the FISPAC research group at the Universidad de Murcia, Spain, where part of this work has been conducted. The work of JJFM has been supported by the Spanish Ministry of Education FPU fellowship AP2008-00919 and grants E024-018 and FIS201124924. The work of ETL has been supported in part by the Ministerio de Educación y Ciencia, grants FIS2011-24924, FIS2015-28521, Universidad de Murcia project E024-018 and Fundacion Seneca (21257/PI/19 and 20949/PI/18). ETL would like to thank the hospitality of the CERN TH Division, Harvard Physics Department and Yukawa Institute (Kyoto University) where part of the research has been conducted. ETL would like to thank ESV for unvaluable support. LB is grateful to Sergio Ferrara for hospitality at the CERN TH Division, where part of this project was initiated. LB and MJD are grateful to Philip Candelas for hospitality at the Mathematical Institute, University of Oxford. MJD is grateful to Marlan Scully for his hospitality in the Institute for Quantum Science and Engineering, Texas A\&M University. MD acknowledges the Leverhulme Trust for an Emeritus Fellowship and the Hagler Institute for Advanced Study at Texas A\&M for a Faculty Fellowship.

\section{A Freudenthal triples: assorted properties}

A summary of some FTS definitions, notation and properties used through this work. See ref. [14] for additional ones and proofs.

$$
\begin{aligned}
\{T(x, y, z), w\} & =2 \Delta(x, y, z, w), \\
x^{\prime} & \equiv T(x, x, x) \equiv T(x), \\
\Delta(x) & \equiv \Delta(x, x, x, x),
\end{aligned}
$$




$$
\begin{aligned}
3\left\{T(x, x, y), y^{\prime}\right\} & =2\{x, y\} \Delta(x, y, y, y), \\
6\left\{T(x, w, y), y^{\prime}\right\} & =\{x, y\} 2 \Delta(w, y, y, y)+\{w, y\} 2 \Delta(x, y, y, y), \\
6\left\{T(x, y, y), y^{\prime}\right\} & =\{x, y\} 2 \Delta(y), \\
9 T((T(x, y, y), y, y) & =-2 \Delta(x, y, y, y) y-\Delta(y) x-\{x, y\} y^{\prime}, \\
T\left(x^{\prime}, x, x\right) & =-\frac{1}{3} \Delta(x) x \\
T\left(x^{\prime}, x^{\prime}, x\right) & =\frac{1}{3} \Delta(x) x^{\prime} \\
\left(x^{\prime}\right)^{\prime} \equiv T\left(x^{\prime}, x^{\prime}, x^{\prime}\right) & =-\Delta^{2}(x) x \\
\Delta\left(x^{\prime}, x, x, x\right) & =0 \\
\Delta\left(x^{\prime}, x^{\prime}, x, x\right) & =\frac{1}{3} \Delta^{2}(x) \\
\Delta\left(x^{\prime}, x^{\prime}, x^{\prime}, x\right) & =0 \\
\Delta\left(x^{\prime}, x^{\prime}, x^{\prime}, x^{\prime}\right) & =\Delta^{3}(x) .
\end{aligned}
$$

Some additional properties related to eq. (3.12):

$$
\text { For } \begin{aligned}
\Delta(x)=0: & T\left(a x+b x^{\prime}\right)=a^{3} x^{\prime}, \\
& , \quad T\left(x^{\prime}\right)=0, \\
\Delta(x)<0: & T\left(\sqrt{|\Delta(x)| x} \pm x^{\prime}\right)=0, \\
\Delta(x)>0: & T\left(\sqrt{\Delta(x)} x \pm x^{\prime}\right)=\mp 2 \Delta(x)^{3 / 2}\left(\sqrt{\Delta(x)} x \mp x^{\prime}\right) .
\end{aligned}
$$

Moreover

$$
\begin{array}{ll}
\Delta(x)<0: & T(x \pm \tilde{x})=0, \\
\Delta(x)>0: & T(x \pm \tilde{x})=\mp 2 \sqrt{\Delta(x)}(x \mp \tilde{x}) .
\end{array}
$$

\section{B More $\mathfrak{T}$-plane properties}

\section{B.1 Complexification of real $\mathfrak{F}$ 's and $\mathfrak{T}$-planes}

The square of the $\Upsilon_{x}$ map, for a fixed $x$, is proportional to the identity $\left(\Delta_{x} \equiv \Delta(x), \epsilon=\right.$ $\operatorname{sgn} \Delta(x))$

$$
\Upsilon_{x}^{2}(y)=-2 \Delta_{x} y
$$

for any $y \in \mathfrak{F}_{y \perp x}$ (see eq. (4.17)). For $\epsilon=1$ this map defines then a local (depending on the point) complex structure, ${ }^{38}$ allowing one to endow, the originally real, $\mathfrak{F}$ with the structure of a complex vector space. We may define its complexification by extension to

$$
\mathfrak{F}^{\mathbb{C}}=\mathfrak{F} \otimes_{\mathbb{R}} \mathbb{C} .
$$

since the algebraic closure of $\mathbb{C}$, the map $\Upsilon_{x}$ is guaranteed to have eigenvalues, proportional to $\pm i$ in the $\epsilon=1$ case.. The space $\mathfrak{F}_{y \perp x}$ can be then split into one dimensional eigenspaces of $\Upsilon_{x}$. We write

$$
\mathfrak{F}_{y \perp x}=\left(\mathfrak{F}_{y \perp x}\right)_{-} \oplus\left(\mathfrak{F}_{y \perp x}\right)_{+}
$$

\footnotetext{
${ }^{38}$ It defines a split complex structure in the $\epsilon=-1$ case.
} 
where, for any $y_{ \pm} \in\left(\mathfrak{F}_{y \perp x}\right)_{ \pm}$,

$$
\Upsilon_{x}\left(y_{ \pm}\right)= \pm i \sqrt{\Delta_{x}} y_{ \pm}
$$

Let us choose normalized eigenvectors (the $\mathfrak{F}_{y \perp x}$ "light-cone" or "null" basis) as follows

$$
y_{ \pm}= \pm i \sqrt{\Delta_{x}} y+\Upsilon_{x}(y) .
$$

It is clear that $\left\{y_{-}, y_{+}\right\}=0$. In addition we have the following properties (see [14], eqs. $(18,19))$,

$$
\begin{aligned}
\Delta\left(y_{ \pm}\right) & =0, \\
\Delta\left(y_{-}, y_{+}, y_{+}, y_{+}\right) & =\Delta\left(y_{+}, y_{-}, y_{-}, y_{-}\right)=0, \\
\left\{\left(y_{ \pm}\right)^{\prime}, y_{\mp}\right\} & =0 .
\end{aligned}
$$

Any element $u \in \mathfrak{F}_{y \perp x}$, such that $u=a y+b \Upsilon_{x}(y)$, can be written as $u=\alpha_{+} y_{+}+\alpha_{-} y_{-}$, with the coordinates in both basis related by $2 \alpha_{\mp}=b \pm i a / \sqrt{\Delta(x)}$. This null basis will be useful in what follows.

We will study now the behaviour of the quadrilinear map $\Delta$ and the antisymmetric form in this plane $\mathfrak{F}_{y \perp x}$.

For any two vectors $u_{1}, u_{2} \in \mathfrak{F}_{y \perp x}$ we have the following properties $\left(u_{i}=a_{i} y+\right.$ $\left.b_{i} \Upsilon_{x}(y)=\alpha_{i+} y_{+}+\alpha_{i-} y_{-}, i=1,2\right)\left(2 \alpha_{i \mp}=b_{i} \pm i a_{i} / \sqrt{\Delta(x)}\right)$,

$$
\begin{aligned}
\left\{u_{1}, u_{2}\right\} & =\left(b_{1} a_{2}-a_{1} b_{2}\right)\left\{\Upsilon_{x}(y), y\right\} \\
& =\left(b_{1} a_{2}-a_{1} b_{2}\right) 6 \Delta(x, x, y, y), \\
\left\{\Upsilon_{x}\left(u_{1}\right), \Upsilon_{x}\left(u_{2}\right)\right\} & =\Delta(x)\left\{u_{1}, u_{2}\right\}, \\
\Delta(u) & =\frac{1}{4 \Delta_{x}^{2}}\left(a^{2}+b^{2} \Delta_{x}\right)^{2} \Delta\left(y_{-}, y_{-}, y_{+}, y_{+}\right), \\
\Delta\left(y_{-}, y_{-}, y_{+}, y_{+}\right) & =4 \Delta(x)^{2} \Delta(y)=4 \Delta\left(\Upsilon_{x}(y)\right) .
\end{aligned}
$$

The first two expressions are obtained by direct computation. To get eq. (B.10), we note that

$$
\begin{aligned}
\Delta(u)= & a_{+}^{4} \Delta\left(y_{+}\right)+a_{-}^{4} \Delta\left(y_{-}\right)+3 \alpha_{+}^{3} \alpha_{-} \Delta\left(y_{-}, y_{+}, y_{+}, y_{+}\right)+ \\
& 3 \alpha_{+} \alpha_{-}^{3} \Delta\left(y_{+}, y_{-}, y_{-}, y_{-}\right)++4 \alpha_{+}^{2} \alpha_{-}^{2} \Delta\left(y_{-}, y_{-}, y_{+}, y_{+}\right) \\
= & 4 \alpha_{+}^{2} \alpha_{-}^{2} \Delta\left(y_{-}, y_{-}, y_{+}, y_{+}\right) \\
= & \frac{1}{4 \Delta_{x}^{2}}\left(a^{2}+b^{2} \Delta_{x}\right)^{2} \Delta\left(y_{-}, y_{-}, y_{+}, y_{+}\right)
\end{aligned}
$$

where we have used eqs. (B.4), (B.5). Finally eq. (B.11) is a particular case of eq. (B.10).

Eq. (B.9) can also be written as (for $\Delta(x) \neq 0$ ),

$$
\left\{\frac{\Upsilon_{x}\left(u_{1}\right)}{\sqrt{|\Delta(x)|}}, \frac{\Upsilon_{x}\left(u_{2}\right)}{\sqrt{|\Delta(x)|}}\right\}=\epsilon\left\{u_{1}, u_{2}\right\} .
$$


As consequence, for $\epsilon=1$, the map

$$
\tilde{\Upsilon}_{x} \equiv \frac{1}{\sqrt{ } \Delta_{x}} \Upsilon_{x}
$$

preserves the bilinear antisymmetric form $\{$,$\} in each \mathfrak{F}_{y \perp x}$ plane. This implies necessarily that $\tilde{\Upsilon}_{x}$ is a symplectic transformation on the plane.

\section{B.2 Maximal rank $\mathfrak{T}$-planes are disjoint}

Let us have two non-degenerate (generated by maximal rank elements) planes $\mathfrak{T}_{x_{0}}, \mathfrak{T}_{x_{1}}$ generated by distinct elements $x_{0}, x_{1}\left(x_{0} \neq x_{1}\right)$. We will show that the two planes are, or the same, or disjoint.

Suppose we can find a common element $y \in \mathfrak{T}_{x_{0}} \cap \mathfrak{T}_{x_{1}}$, this implies that a) the signs of $\Delta\left(x_{0}\right), \Delta\left(x_{1}\right)$ are the same and b) also $y^{\prime} \equiv T(y) \in \mathfrak{T}_{x_{0}} \cap \mathfrak{T}_{x_{1}}$. Then, we can find coefficients $a_{i}, b_{i}, \alpha_{i}, \beta_{i}$ such that

$$
\begin{aligned}
y & =a_{0} x_{0}+b_{0} x_{0}^{\prime}=a_{1} x_{1}+b_{1} x_{1}^{\prime}, \\
y^{\prime} & =\alpha_{0} x_{0}+\beta_{0} x_{0}^{\prime}=\alpha_{1} x_{1}+\beta_{1} x_{1}^{\prime} .
\end{aligned}
$$

The coefficients $\alpha_{i}, \beta_{i}$ are given in terms of the $a_{i}, b_{i}$ by eq. (3.4). Inserting the values of these coefficients we can see that these equations are invertible as long as

$$
\Delta(y) / \Delta\left(x_{1}\right)=\Delta(y) / \Delta\left(x_{0}\right)=\left(a_{0}^{2}+b_{0}^{2} \Delta_{0}\right)^{2}=\left(a_{1}^{2}+b_{1}^{2} \Delta_{1}\right)^{2} \neq 0 .
$$

If $\Delta(y) \neq 0$ the equations are invertible: we can write any pair $\left(x_{0}, x_{0}^{\prime}\right)$ or $\left(x_{1}, x_{1}^{\prime}\right)$ as linear combinations of $y, y^{\prime}$. The existence of such linear combinations shows that $\mathfrak{T}_{y}=\mathfrak{T}_{x_{0}}=\mathfrak{T}_{x_{1}}$.

If the common element is not of maximal rank then the situation is different. If $\Delta(y)=0$, the equations are not invertible but then necessarily both $\Delta\left(x_{0}\right)<0, \Delta\left(x_{1}\right)<0$. $y$ is a "null" or "light-cone" element, it can be seen it is then proportional to one of the two vectors

$$
\pm \sqrt{\left|\Delta\left(x_{0}\right)\right|} x_{0}+x_{0}^{\prime}
$$

and one of the two

$$
\pm \sqrt{\left|\Delta\left(x_{1}\right)\right|} x_{1}+x_{1}^{\prime} .
$$

We can use these last expressions to arrive to:

$$
\pm \sqrt{\left|\Delta\left(x_{0}\right)\right|} x_{0}+x_{0}^{\prime} \propto \pm \sqrt{\left|\Delta\left(x_{1}\right)\right|} x_{1}+x_{1}^{\prime} .
$$

"Some" elements of $\mathfrak{T}_{x_{0}}$ (a one dimensional subset of them) can be written as linear combination of those of $\mathfrak{T}_{x_{1}}$.

In the case of degenerate planes (generated by non-maximal rank elements), one can show in the same way that the T-"lines" $\mathfrak{T}_{x_{0}}, \mathfrak{T}_{x_{1}}$, corresponding to elements $x_{0}, x_{1}$ with $\Delta\left(x_{0}\right)=\Delta\left(x_{1}\right)=0$ are either identical (if $x_{0}^{\prime}=x_{1}^{\prime}=0$ or $x_{0}^{\prime} \propto x_{1}^{\prime}$ ) or disjoint (if $x_{0}^{\prime} \not \subset x_{1}^{\prime}$ ). 


\begin{tabular}{|c|c|c|l|c|}
\hline$R k\left(x_{0}\right)$ & & Cases & & $\operatorname{Rank}(\mathrm{x})$ \\
\hline 4 & $\Delta_{0}>0$ & $\Delta(x)>0$ & & 4 \\
4 & $\Delta_{0}<0$ & $\Delta(x)<0$ & & 4 \\
& & $\Delta(x)=0$ & $x^{\prime}=0, x \propto x_{ \pm}, \Upsilon_{x+}\left(x_{-}\right) \neq 0$, & 2 \\
3 & $\Delta_{0}=0, x_{0}^{\prime} \neq 0$ & $x \not \subset x_{0}^{\prime}$ & $x^{\prime} \propto x_{0}^{\prime}$, & 3 \\
& & $x \propto x_{0}^{\prime}$ & $T\left(x^{\prime}\right)=0$, & 1,2 \\
1,2 & $\Delta_{0}=0, x_{0}^{\prime}=0$ & $\Delta(x)=0$ & $x^{\prime}=0, x \propto x_{0}, \Upsilon_{x} \propto \Upsilon_{x 0}$, & $=\operatorname{Rank}\left(x_{0}\right)$ \\
\hline
\end{tabular}

Table 2. Relation of $\operatorname{rank}(x)$, the rank of any element $x$ in the $\mathfrak{T}$-plane $\mathfrak{T}_{0}$ generated by $x_{0}$.

\section{B.3 Rank on the $\mathfrak{T} / \mathfrak{F}$-plane}

Let it be a generic vector $x_{0}$ of a given rank and its associated (possible degenerate) $\mathfrak{T}$-plane $\mathfrak{T}_{x_{0}} \equiv \mathfrak{T}_{0}$ ( we write $\Delta_{0}=\Delta\left(x_{0}\right)$ ). We are interested in studying what it can be said about the rank of any element $x \in \mathfrak{T}_{0}$ on the plane. Let us consider the different cases.

Let us assume then a nonzero rank and consider the following possibilities:

A) If $\Delta_{0}>0$ then, by eq. (3.9), $\Delta(x)>0$, and $\operatorname{Rank}(x)=\operatorname{Rank}\left(x_{0}\right)=4, \forall x \in \mathfrak{T}_{0}$.

B) If $\Delta_{0}<0$ then, by eq. (3.9), we have two possibilities: $\Delta(x)<0$ and then $\operatorname{Rank}(x)=$ 4 or $\Delta(x)=0$.

If $\Delta(x)=0$ then $x$ is a null vector, it belongs to the light-cone generated by $x_{ \pm}=$ $\pm \sqrt{\left|\Delta\left(x_{0}\right)\right|} x_{0}+x_{0}^{\prime}$, then, by eq. (3.4), $x^{\prime}=T(x)=0$. For the sake of concreteness, let us take $x=x_{+}$. We can find at least one element, its associated element $x_{-}$, such that

$$
\Upsilon_{x_{+}}\left(x_{-}\right) \propto-8\left|\Delta_{0}\right| \sqrt{\left|\Delta\left(x_{0}\right)\right|} x_{+} \neq 0
$$

similarly would happen if we start by assuming $x=x_{-}$. We arrive to the conclusion that $\operatorname{Rank}(x)=2$ in this case.

C) If $\Delta_{0}=0\left(\operatorname{Rank}\left(x_{0}\right)=1,2,3\right)$ then, by eq. (3.9), $\Delta(x)=0$. We are confronted with two self-excluding possibilities:

C1)If $x_{0}^{\prime} \neq 0\left(\operatorname{Rank}\left(x_{0}\right)=3\right)$ then $x^{\prime}=a^{3} x_{0}^{\prime} \neq 0(a \neq 0)$ and $\operatorname{Rank}(x)=3$. If $a=0$ then $T\left(x^{\prime}\right)=0$ and the rank of $x$ is $R=1,2$.

C2)If $x_{0}^{\prime}=0\left(\operatorname{Rank}\left(x_{0}\right)=1,2\right)$ then $x^{\prime} \propto x_{0}^{\prime}=0, x \propto x_{0} . \Upsilon_{x}(y) \propto \Upsilon_{x 0}(y)$ and $\operatorname{Rank}(x)=\operatorname{Rank}\left(x_{0}\right)$ in this case.

The results are summarized in table 2 . Similar results are obtained for the $\mathfrak{F}$-plane.

\section{B.4 The $\mathfrak{T}$ - or $\mathfrak{F}$-plane as a (quadratic) sub-FTS system: Euclidean and hy- perbolic planes}

Let us fix a maximal rank element $x_{0}\left(\Delta\left(x_{0}\right) \neq 0\right)$ and its $\mathfrak{T}$-plane $\mathfrak{T}_{x_{0}} \equiv \mathfrak{T}_{0}$. As a consequence of eq. (3.9), the restriction of the quartic map $\Delta$ to $\mathfrak{T}_{0}$ can be written in terms of a symmetric bilinear form $I_{2}$ as follows

$$
\Delta_{\mathfrak{T}_{0}}(x)=\frac{\epsilon_{0}}{4} I_{2}^{2}(x), \quad\left(\forall x \in \mathfrak{T}_{0}\right),
$$


where $\epsilon_{0}=\operatorname{sgn} \Delta\left(x_{0}\right)$ (we call $\epsilon_{0}$ the "signature of $\mathfrak{T}_{0}$ ", it does not depend on the plane maximal rank element chosen as generator).

Choosing a basis on the plane and coordinates with respect it $\left(x=x^{I} e_{I},(I=1,2)\right.$, $\left.e_{1} \equiv x_{0}, e_{2} \equiv x_{0}^{\prime}\right)$, the bilinear form is given by

$$
I_{2}(x, y) \equiv x^{I} g_{I J} y^{J}=2 \sqrt{\left|\Delta_{0}\right|}\left(x^{1} y^{1}+x^{2} y^{2} \Delta_{0}\right) .
$$

Then the quadratic form

$$
I_{2}(x)=2 \sqrt{\left|\Delta_{0}\right|}\left(\left(x^{1}\right)^{2}+\left(x^{2}\right)^{2} \Delta_{0}\right) .
$$

The full quadrilinear map restricted to the plane is then of the form (for generic vectors $\left.x, y, z, w \in \mathfrak{T}_{0}\right)$

$$
\Delta_{T_{0}}(x, y, z, w)=\frac{\epsilon_{0}}{12}\left(I_{2}(x, y) I_{2}(z, w)+I_{2}(x, z) I_{2}(y, w)+I_{2}(x, w) I_{2}(y, z)\right) .
$$

To arrive to this expression we have used the multi-linearity of $\Delta$ and the properties $\Delta\left(x, x, x, x^{\prime}\right)=\Delta\left(x, x^{\prime}, x^{\prime}, x^{\prime}\right)=0$ (see properties in section A). In the case of $\Delta_{0}=0$ the expressions reduce trivially to $I_{2} \equiv 0$.

We can quickly convince us that the $\mathfrak{T}_{0}$ plane is itself a two dimensional FTS of quadratic (or degenerate) type (see [58]) with a characteristic "signature ", $\epsilon_{0}$, and whose symmetric quadrilinear and antisymmetric bilinear forms are those inherited from the original FTS.

Restricted to the T-plane the quadrilinear and bilinear forms acquire simple expressions. The antisymmetric bracket is

$$
\{x, y\}_{\mathfrak{T}_{0}} \equiv x^{I} \omega_{I J} y^{J}=\omega_{12}\left(x^{1} y^{2}-x^{2} y^{1}\right) .
$$

with

$$
\omega_{12}=\left\{x_{0}, x_{0}^{\prime}\right\}=-2 \Delta_{0} .
$$

We define the matrix $S=\left(S_{I J}\right)$ (see [58]), such that

$$
S_{J}^{I}=\left(g^{-1}\right)^{I K} \omega_{K J},
$$

then

$$
S^{2}=-\epsilon_{0} I \text {. }
$$

The explicit form of the matrix $S$ is

$$
\left(S_{J}^{I}\right)=\epsilon_{0}\left(\begin{array}{cc}
0 & -\sqrt{\left|\Delta_{0}\right|} \\
\frac{1}{\sqrt{\left|\Delta_{0}\right|}} & 0
\end{array}\right),
$$

The trilinear $T$ map, the F-dual maps restricted to $\mathfrak{T}_{0}$ are given by, in component form [58]

$$
\begin{aligned}
T(x, y, z) & =\frac{1}{6}\left(I_{2}(x, y) \hat{S}(z)+I_{2}(x, z) \hat{S}(y)+I_{2}(y, z) \hat{S}(x)\right), \\
x^{\prime} & =\frac{1}{2} I_{2}(x) \hat{S}(x) \\
\tilde{x} & =\eta(x) \hat{S}(x) .
\end{aligned}
$$


where it has been used the linear map defined by $\hat{S}(x) \equiv S^{I}{ }_{J} x^{J} e_{I}$ and where

$$
\eta(x) \equiv \operatorname{sgn}\left(I_{2}(x)\right)=\operatorname{sgn}\left(\left(x^{1}\right)^{2}+\epsilon_{0}\left(x^{2}\right)^{2}\left|\Delta_{0}\right|\right) .
$$

If $\epsilon_{0}=1$ then $\eta(x)=1, \forall x \in \mathfrak{T}_{0}$.

In summary any non-degenerate $T$ (or $F$ ) plane can be considered as a two-dimensional quadratic FTS system, the three FTS axioms, see section 2, are trivially satisfied by the $\mathfrak{T}_{0}$-restricted maps $\Delta_{T},\{\}_{T}, T_{T}\left(\Delta_{F},\{\}_{F}, T_{F}\right)$.

Euclidean, hyperbolic $\mathfrak{T}$-planes. The signature of the $I_{2}$ bilinear form coincides with the signature of the $\mathfrak{T}_{0}$ plane, the sign of $\Delta\left(x_{0}\right)$ with $x_{0}$ any maximal rank element in the plane. Thus $I_{2}$ defines an Euclidean or a Minkowskian $R^{1,1}$ (hyperbolic or split-complex) structure on the $\mathfrak{T}$-plane according to it.

Let us focus on the second case. Endowed by the metric $I_{2}, \mathfrak{T}_{0}$ becomes a Minkowski plane. The set of all transformations of the hyperbolic plane which preserve the $I_{2}$ form is the group $O(1,1)$. This group consists of the hyperbolic subgroup $S O^{+}(1,1)$, combined with four discrete reflections given by

$$
x \rightarrow \pm x, x^{\prime} \rightarrow \pm x^{\prime} .
$$

Using standard notation, we say that a non-zero vector $x \in \mathfrak{T}_{0}$ is spacelike if $I_{2}(x)>0$, lightlike, or null, if $I_{2}(x)=0$ and timelike if $I_{2}(x)<0$.

The T operation, $x \rightarrow x^{\prime}$, change the character of the vector. The vector $x^{\prime}$ is " $I_{2^{-}}$ orthogonal" (or "simultaneous events") to $x$. One can check that [58],

$$
\begin{aligned}
I_{2}\left(x, x^{\prime}\right) & =\frac{1}{2} I_{2}(x) I_{2}(x, \hat{S} x) \\
& =\frac{1}{2} I_{2}(x)\{x, x\}=0 .
\end{aligned}
$$

The relation $x^{\prime}= \pm x$ is true if and only if $x$ is lightlike; $x^{\prime}$ is timelike (resp. spacelike) if $x$ is spacelike (resp. timelike):

$$
\begin{aligned}
x \text { :lightlike } & \longleftrightarrow x^{\prime}: \text { lightlike }, \quad x^{\prime}= \pm x, \\
x: \text { timelike }(\text { spacelike }) & \left.\longleftrightarrow x^{\prime}: \text { spacelike (timelike }\right)
\end{aligned}
$$

\section{B.5 Coordinates in the $\mathfrak{T}$-plane. Orbits}

On the "Euclidean" $\mathfrak{F}$-plane, by eq. (5.1), the orbits of the exponential of the $\Upsilon$ map for $\epsilon=1$ are closed circles. Let $e_{i}=(e, \tilde{e})$ be the orthonormal basis given by $e \equiv x /|\Delta(x)|^{1 / 4}$. Then

$$
\Delta(e)=\epsilon, \quad\left\{e_{i}, e_{j}\right\}=-2 \varepsilon_{i j},
$$

For a generic $u \in \mathfrak{F}_{x}$ expressed in this basis, $u=u^{1} e+u^{2} \tilde{e}$, we arrive to, applying eq. (5.6),

$$
\Delta(x)>0: e^{\bar{\Upsilon}_{x}}(u)=\left(\cos (\theta) u^{1}-\sin (\theta) u^{2}\right) e+\left(\sin (\theta) u^{1}+\cos (\theta) u^{2}\right) \tilde{e} .
$$


Whereas, on a "Minkowskian" plane, with $\epsilon=-1$, the same orbits are hyperbolic, they are given by

$$
\Delta(x)<0: e^{\bar{\Upsilon}_{x}}(u)=\left(\cosh (\theta) u^{1}-\sinh (\theta) u^{2}\right) e+\left(-\sinh (\theta) u^{1}+\cosh (\theta) u^{2}\right) \tilde{e} .
$$

The case $\epsilon=-1$ with $u^{1}= \pm u^{2}$ corresponds to null vectors with orbits made by $\pi / 4$ rays:

$$
\Delta(x)<0, u^{1}= \pm u^{2}: e^{\bar{\Upsilon}_{x}}(u)= \pm e^{\mp \theta} u^{2}(e \pm \tilde{e}) .
$$

\section{B.6 "Light-cone" coordinates in the T-plane}

It is of interest to define a basis formed by the vectors

$$
e_{ \pm}=x \pm \tilde{x}
$$

For any $y \in F$ we define coordinates $\alpha_{ \pm}$such that

$$
y=\alpha_{+} e_{+}+\alpha_{-} e_{-} .
$$

In terms of these coordinates the quadrilinear map is given by

$$
\Delta(y)=\left(\left(\alpha_{+}+\alpha_{-}\right)^{2}+\epsilon\left(\alpha_{+}-\alpha_{-}\right)^{2}\right)^{2} \Delta(x) .
$$

For $\Delta(x)<0$ ( $\epsilon=-1$ in the previous expression) the basis thus defined is a null basis $\left(\Delta\left(e_{ \pm}\right)=0\right), \alpha_{ \pm}$are "null" or "light-cone" coordinates, and then

$$
\begin{aligned}
& \Delta(y)=\left(4 \alpha_{+} \alpha_{-}\right)^{2} \Delta(x), \quad(\epsilon=-1), \\
& \Delta(y)=4\left(\alpha_{+}+\alpha_{-}\right)^{2} \Delta(x), \quad(\epsilon=+1) .
\end{aligned}
$$

Similar coordinates will be defined on a $\mathfrak{T}$-plane.

\section{B.7 The general exponential map}

Let us consider now the action on the orthogonal complement of a given element $x \in \mathfrak{F}$. For any $y \in \mathfrak{T}_{x}^{\perp}$ ( that means $\{x, y\}=0,\left\{x^{\prime}, y\right\}=0$, see eq. (4.2)), we have also seen that $\Upsilon_{x}(y) \in \mathfrak{T}_{x}^{\perp}$. In conclusion, for any $y \in \mathfrak{T}_{x}^{\perp}$ the action of successive applications of $\Upsilon_{x}$ is restricted to lie on $\mathfrak{F}_{y \perp x}, \Upsilon_{x}^{n}(y)$. The orbit of any $y \in \mathfrak{T}_{x}^{\perp}$ under $\sigma_{x}(\theta)$ lies completely on $\mathfrak{F}_{y \perp x}$. This can be seen from eq. (4.18) and eq. (4.19). These equations allow to explicitly compute the exponential map by summing an exponential series, for any rank-4 $x$,

$$
\left(\exp \theta \Upsilon_{x}\right)(y)=\sum_{k=0} \frac{\left(\sqrt{-\Delta_{x}} \theta\right)^{2 k}}{(2 k) !} y+\frac{1}{\sqrt{-\Delta_{x}}} \sum_{k=0} \frac{\left(\sqrt{-\Delta_{x}} \theta\right)^{2 k+1}}{(2 k+1) !} \Upsilon_{x}(y) .
$$

A similar series is obtained for $\left(\exp \theta \Upsilon_{x}\right)\left(\Upsilon_{x}(y)\right)$.

Summing the series, the exponential of the "normalized" map in the orthogonal plane is fully determined by the expressions $\left(\bar{\Upsilon}_{x} \equiv \Upsilon_{x} /(\sqrt{|\Delta(x)|}, \epsilon=\operatorname{sgn} \Delta(x))\right.$

$$
\begin{aligned}
\left(\exp \theta \overline{\bar{\Upsilon}}_{x}\right)(y) & =\cos (\sqrt{\epsilon} \theta) y+\epsilon \sqrt{\epsilon} \sin (\sqrt{\epsilon} \theta) \overline{\bar{\Upsilon}}_{x}(y), \\
\left(\exp \theta \overline{\bar{\Upsilon}}_{x}\right)\left(\overline{\bar{\Upsilon}}_{x}(y)\right) & =-\sqrt{\epsilon} \sin (\sqrt{\epsilon} \theta) y+\cos (\sqrt{\epsilon} \theta) \overline{\bar{\Upsilon}}_{x}(y) .
\end{aligned}
$$


The geometrical character of the orbits of the exponential of the $\Upsilon_{x}$ map in the $\mathfrak{F}_{x}^{\perp}$ plane solely depends on $\epsilon$, the sign of $\Delta(x)$, and not, for example, on the signature of $y$. They are closed (circles or ellipses) or hyperbolic, respectively for $\epsilon=1$ or -1 .

It can be explicitly checked that

$$
\left\{e^{\theta \bar{\Upsilon}_{x}}(y), e^{\theta \bar{\Upsilon}_{x}}\left(\bar{\Upsilon}_{x}(y)\right)\right\}=\left\{y, \bar{\Upsilon}_{x}(y)\right\}
$$

We have also, according to eq. (4.27)

$$
\Delta\left(\tilde{\Upsilon}_{x}^{n}(y)\right)=\Delta(y)
$$

Let us compute now, for a fixed element $x$, the exponential map $\exp \theta \bar{\Upsilon}_{x}$ on a generic FTS element $z$, not necessarily on the orthogonal complement $\mathfrak{T}_{x}^{\perp}$. For that purpose, first we decompose the element on its $\mathfrak{F}_{x}$ parallel and orthogonal components

$$
z=z_{\|}+z_{\perp}
$$

Without loss of generality we can assume that $z_{\|}=x$ (if it is not so, we simply realign the $\mathfrak{F}$-plane by choosing $z_{\|}$as the defining element of the plane: $\left.\mathfrak{F}_{x} \equiv \mathfrak{F}_{z \|}\right)$. Then $z=x+z_{\perp}$. The action of any power of $\Upsilon_{x}$ on $z$ is, by linearity,

$$
\Upsilon_{x}^{n}\left(x+z_{\perp}\right)=\Upsilon_{x}^{n}(x)+\Upsilon_{x}^{n}\left(z_{\perp}\right)
$$

with $\Upsilon_{x}^{n}(x) \in \mathfrak{F}_{x}$ and $\Upsilon_{x}^{n}\left(z_{\perp}\right) \in \mathfrak{F}_{x}^{\perp}$.

As a consequence, the exponential of the $\Upsilon_{x}$ (or $\bar{\Upsilon}_{x}$ ) is of the form ( for $z=x+z_{\perp}$ ):

$$
\left(\exp \theta \bar{\Upsilon}_{x}\right)(z)=\left(\exp \theta \bar{\Upsilon}_{x}\right)(x)+\left(\exp \theta \bar{\Upsilon}_{x}\right)\left(z_{\perp}\right)
$$

where any of summands is computed independently using the corresponding relations (eqs. (5.6) and (5.7) for $\mathfrak{F}_{x}$, or, (B.41) and (B.42) for $\mathfrak{F}_{x}^{\perp}$ ). Putting together these relations, one arrives to

$$
\begin{aligned}
e^{\theta \bar{\Upsilon}_{x}}(z)= & \cos \left(\sqrt{\epsilon} \frac{\theta}{3}\right) z_{\perp}+3 \epsilon \sqrt{\epsilon} \sin \left(\sqrt{\epsilon} \frac{\theta}{3}\right) \bar{\Upsilon}_{x}\left(z_{\perp}\right) \\
& +\cos (\sqrt{\epsilon} \theta) x+\epsilon \sqrt{\epsilon} \sin (\sqrt{\epsilon} \theta) \tilde{x} .
\end{aligned}
$$

In particular, for any $z_{\perp}$ in the orthogonal space, the vector

$$
w=z_{\perp} \pm 3 \epsilon \sqrt{-\epsilon} \bar{\Upsilon}_{x}\left(z_{\perp}\right)
$$

of the exponential map (strictly, we have to deal with a complexified FTS for $\epsilon=-1$, see subsection B.1):

$$
\left(\exp \theta \bar{\Upsilon}_{x}\right) w=e^{\mp \sqrt{-\epsilon} \frac{\theta}{3}} w .
$$

The map $\exp \left(\theta \bar{\Upsilon}_{x}\right)$ over a generic element $y$ in the FTS can be obtained from the previous formula performing a suitable rotation in the $\mathfrak{F}_{x}$ plane bringing $x$ to a generic $y_{\|}$, 
and thus $\tilde{x}$ to $\widetilde{y_{x}}$ :

$$
\begin{aligned}
\exp \left(\theta \bar{\Upsilon}_{x}\right)(y)= & \cos \left(\sqrt{\varepsilon} \frac{\theta}{3}\right) y+3 \varepsilon \sqrt{\varepsilon} \sin \left(\sqrt{\varepsilon} \frac{\theta}{3}\right) \bar{\Upsilon}_{x}(y) \\
& +\left[\cos (\sqrt{\varepsilon} \theta)-\cos \left(\sqrt{\varepsilon} \frac{\theta}{3}\right)\right] y_{\|_{x}} \\
& +\sqrt{\varepsilon}\left[\sin (\sqrt{\varepsilon} \theta)-3 \eta \sin \left(\sqrt{\varepsilon} \frac{\theta}{3}\right)\right] \widetilde{y_{\|_{x}}}
\end{aligned}
$$

where $\eta=\operatorname{sgn}\left(\{\tilde{x}, y\}^{2}+\varepsilon\{x, y\}^{2}\right)$. Explicitly, for $\epsilon=1$, one obtains

$$
\begin{aligned}
e^{\theta \bar{\Upsilon}_{x}}(y)= & \cos \left(\frac{\theta}{3}\right) y+3 \sin \left(\frac{\theta}{3}\right) \bar{\Upsilon}_{x}(y) \\
& +\left[\cos (\theta)-\cos \left(\frac{\theta}{3}\right)\right] y_{\| x}+\left[\sin (\theta)-3 \sin \left(\frac{\theta}{3}\right)\right] \widetilde{y_{\| x}}
\end{aligned}
$$

and similarly for $\epsilon=-1$. Here we have introduced the projections of the generic vector $y$ and its F-dual. ${ }^{39}$ By reordering the terms in eq. (B.51), one can also write

$$
\begin{aligned}
e^{\theta \bar{\Upsilon}_{x}}(y)= & \cos \left(\frac{\theta}{3}\right) y_{\perp}+3 \sin \left(\frac{\theta}{3}\right)\left(\bar{\Upsilon}_{x}(y)-\widetilde{y_{\| x}}\right) \\
& +\cos (\theta) y_{\| x}+\sin (\theta) \widetilde{y_{\| x}} .
\end{aligned}
$$

We see that the orbit of any generic element of the FTS under a $\sigma_{x}(\theta)$, for fixed $x$, lies on a 4-dimensional hyperplane spanned by (taking into account that $x^{\prime} \sim \Upsilon_{x}(x)$ )

$$
\left(x, \Upsilon_{x}(x), z_{\perp}, \Upsilon_{x}\left(z_{\perp}\right)\right)
$$

or

$$
\exp \theta \Upsilon_{x}: \mathfrak{F}_{x} \oplus \mathfrak{F}_{\left(z_{\perp}, \Upsilon_{x}\left(z_{\perp}\right)\right)} \rightarrow \mathfrak{F}_{x} \oplus \mathfrak{F}_{\left(z_{\perp}, \Upsilon_{x}\left(z_{\perp}\right)\right)}
$$

or

$$
\exp \theta \Upsilon_{x}:\left\{\begin{array}{l}
\mathfrak{F}_{x} \rightarrow \mathfrak{F}_{x}, \\
\mathfrak{F}_{\left(z_{\perp}, \Upsilon_{x}\left(z_{\perp}\right)\right)} \rightarrow \mathfrak{F}_{\left(z_{\perp}, \Upsilon_{x}\left(z_{\perp}\right)\right)} .
\end{array}\right.
$$

It is easy to show some explicit examples of the application of eq. (B.47). For $y=x+y_{\perp}$, $w=\exp \theta \bar{\Upsilon}_{x}(y)(\epsilon=1)$ where it is obvious the $6 \pi$ periodicity behaviour of the exponential map for this signature:

$$
\begin{array}{ll}
\theta=0, & w=y \\
\theta=3 \pi / 2, & w=-\tilde{x}+3 \bar{\Upsilon}_{x}\left(y_{\perp}\right) \\
\theta=3 \pi, & w=-y \\
\theta=9 \pi / 2, & w=\tilde{x}-3 \bar{\Upsilon}_{x}\left(y_{\perp}\right) \\
\theta=6 \pi, & w=y .
\end{array}
$$

One can explicitly check that $\Delta(w)=\Delta(y)$ in all the cases.

\footnotetext{
${ }^{39}$ Note that, comparing with eqs. (4.6)-(4.11), $P_{z}(y) \equiv y_{\|_{z}}=\frac{1}{2 \sqrt{|\Delta(z)|}}[\{\tilde{z}, y\} z-\{z, y\} \tilde{z}], Q_{z}(y) \equiv \widetilde{y_{\| z}}=$ $\frac{\eta}{2 \sqrt{|\Delta(z)|}}[\{\tilde{z}, y\} \tilde{z}+\varepsilon\{z, y\} z]$.
} 


\section{FTS Darboux canonical form: a foliation on $\mathfrak{F}_{y \perp x}$ planes}

In the previous sections we have seen how it is natural to define structures on the FTS space as $\mathfrak{F}$-planes and $\mathfrak{F}_{x}$, their orthogonal complement $\mathfrak{F}_{x}^{\perp}$. Within any $\mathfrak{F}_{x}^{\perp}$ it results also natural to define planes $\mathfrak{F}_{y \perp x}$ closed under the action of the $\Upsilon_{x}$ map. This decomposition of the $\mathfrak{F}_{x}^{\perp}$ space can be performed in a systematic way providing a natural canonical form for any FTS, similar to the Darboux canonical form of any symplectic space.

The orthogonal space $\mathfrak{F}_{x}^{\perp}$ can be further decomposed in 2-dimensional subpaces orthogonal with respect to the antisymmetric bilinear form $\{$,$\} .$

Given a fixed initial element $x_{0}$ of maximal rank, let us first define for convenience the shorthand notation

$$
\dot{x} \equiv \Upsilon_{x_{0}}(x)
$$

for the fixed element $x_{0}$. In particular $\dot{x}_{0}=3 x_{0}^{\prime}=3 T\left(x_{0}\right)$. We will construct on continuation a series of mutually orthogonal vectors iterating the procedure used before eq. (4.6) in a sort of modified Gram-Schmidt procedure. Let us initially assume a number of pairs, formed by some vectors and their transforms, $\left(x_{0}, \dot{x}_{0}\right),\left(x_{1}, \dot{x}_{1}\right), \ldots\left(x_{n-1}, \dot{x}_{n-1}\right)$, which are already mutually orthogonal, that means (for $i, j=0, n-1$ )

$$
\begin{aligned}
& \left\{x_{i}, x_{j}\right\}=0, \\
& \left\{x_{i}, \dot{x}_{j}\right\}=c_{i} \delta_{i j} .
\end{aligned}
$$

Where $c_{i}$ are nonzero constants.We now extend this set of pairs by iteration. We show that it is possible to find a pair $\left(x_{n}, \dot{x}_{n}\right)$ orthogonal to the previous ones. Let us take an arbitrary vector $z$ and decompose in parallel and orthogonal parts with respect all these vectors, $z=z_{\|}+z_{\perp}$. The parallel part is easily computed, it is the sum of the parallel parts to each of the individual pairs. It is given by eq. (4.6)

$$
z_{\|}=\sum_{i=0, n-1} z_{\| x_{i}}=\sum_{i=0, n-1} \frac{1}{\left\{\dot{x}_{i}, x_{i}\right\}}\left|\begin{array}{cc}
x_{i} & \dot{x}_{i} \\
\left\{x_{i}, z\right\} & \left\{\dot{x}_{i}, z\right\}
\end{array}\right| .
$$

Obviously, the $z_{\|}$defined in this way is on the subspace generated by $x_{i}, \dot{x}_{i},(i=0, n-1)$. The vector $z_{\perp}=z-z_{\|}$is orthogonal by construction to all the subspace,

$$
\begin{aligned}
& \left\{x_{i}, z_{\perp}\right\}=0, \\
& \left\{\dot{x}_{i}, z_{\perp}\right\}=0 .
\end{aligned}
$$

It is also straightforward to show that $\left(z_{\perp}\right)^{*} \equiv \Upsilon_{x_{0}}\left(z_{\perp}\right)=3 T\left(x_{0}, x_{0}, z_{\perp}\right)$ is also orthogonal to the full set:

$$
\begin{aligned}
\left\{\Upsilon_{x_{0}}\left(z_{\perp}\right), x_{i}\right\} & \left.=\left\{3 T\left(x_{0}, x_{0}, z_{\perp}\right), x_{i}\right\}=\left\{3 T\left(x_{0}, x_{0}, x_{i}\right), z_{\perp}\right)\right\} \\
& =\left\{\Upsilon_{x_{0}}\left(x_{i}\right), z_{\perp}\right\}=0, \\
\left\{\Upsilon_{x_{0}}\left(z_{\perp}\right), \dot{x}_{i}\right\} & =9\left\{T\left(x_{0}, x_{0}, z_{\perp}\right), T\left(x_{0}, x_{0}, x_{i}\right)\right\}=-C_{i} \Delta\left(x_{0}\right)\left\{x_{0}, z_{\perp}\right\}=0 .
\end{aligned}
$$


In the last line $C_{0}=3, C_{i}=1,(i=1, n-1)$. So $\left(z_{\perp},\left(z_{\perp}\right)\right)$ is the pair we were looking for, we redefine

$$
\begin{aligned}
& x_{n} \equiv z_{\perp} \\
& \dot{x}_{n} \equiv 3 T\left(x_{0}, x_{0}, z_{\perp}\right)=\left(\dot{z}_{\perp}\right) .
\end{aligned}
$$

The process is iterated as long as we exhaust the dimensionality of the vector space $(n=N)$ or we cannot find vectors with non trivial pairs, $\left(x_{i}, \dot{x}_{i}\right) \neq 0$.

In this way we reduce the symplectic form to a canonical Darboux form. In the basis formed by the vectors $\left(x_{0}, \dot{x}_{0}, x_{1}, \dot{x}_{1}, \ldots\right)$ the the symplectic form is expressed by the matrix

$$
\Omega=\left[\begin{array}{cccccc}
0 & \lambda_{1} & & & \\
-\lambda_{1} & 0 & & & \\
& & \ddots & & \\
& & & 0 & \lambda_{N} \\
0 & & & -\lambda_{N} & 0
\end{array}\right]
$$

where

$$
\lambda_{i}=\left\{x_{i}, \dot{x}_{i}\right\}
$$

\section{The reduced $\mathfrak{F}\left(J_{3}\right)$ case: explicit expressions}

We present here some explicit formulas used in section 8. By exploiting the results in appendix D of [97], one can compute the components of $T(x, y, z)_{M}$ eq. (8.9) in the $4 D / 5 D$ special coordinates' symplectic frame, characterizing every reduced FTS. Using obvious notation (see section 8), these components read:

$$
\begin{aligned}
T(x, y, z)_{0}= & -\frac{1}{3} d^{i j k} x_{i} y_{j} z_{k}-\frac{1}{3}\left(x^{0} y_{0} z_{0}+x_{0} y^{0} z_{0}+x_{0} y_{0} z^{0}\right) \\
& -\frac{1}{6}\left[\left(x^{i} y_{i}+x_{i} y^{i}\right) z_{0}+\left(x^{i} z_{i}+x_{i} z^{i}\right) y_{0}+\left(y^{i} z_{i}+y_{i} z^{i}\right) x_{0}\right] \\
T(x, y, z)_{i}= & \frac{1}{3} d_{i j k}\left(x_{0} y^{j} z^{k}+x^{j} y_{0} z^{k}+x^{j} y^{k} z_{0}\right)+\frac{1}{3} d_{i j m} d^{m k l}\left(x^{j} y_{k} z_{l}+x_{k} y^{j} z_{l}+x_{k} y_{l} z^{j}\right) \\
& -\frac{1}{6}\left[\left(x^{j} y_{j}+x_{j} y^{j}\right) z_{i}+\left(x^{j} z_{j}+x_{j} z^{j}\right) y_{i}+\left(y^{j} z_{j}+y_{j} z^{j}\right) x_{i}\right] \\
& -\frac{1}{6}\left[\left(x^{0} y_{0}+x_{0} y^{0}\right) z_{i}+\left(x^{0} z_{0}+x_{0} z^{0}\right) y_{i}+\left(y^{0} z_{0}+y_{0} z^{0}\right) x_{i}\right] ; \\
T(x, y, z)^{0}= & \frac{1}{3} d_{i j k} x^{i} y^{j} z^{k}-\frac{1}{3}\left(x^{0} y^{0} z_{0}+x^{0} y_{0} z^{0}+x_{0} y^{0} z^{0}\right) \\
& -\frac{1}{6}\left[\left(x^{i} y_{i}+x_{i} y^{i}\right) z^{0}+\left(x^{i} z_{i}+x_{i} z^{i}\right) y^{0}+\left(y^{i} z_{i}+y_{i} z^{i}\right) x^{0}\right] ; \\
T(x, y, z)^{i}= & -\frac{1}{3} d^{i j k}\left(x^{0} y_{j} z_{k}+x_{j} y^{0} z_{k}+x_{j} y_{k} z^{0}\right)+\frac{1}{3} d_{k l m} d^{m i j}\left(x_{j} y^{k} z^{l}+x^{k} y_{j} z^{l}+x^{k} y^{l} z_{j}\right) \\
& -\frac{1}{6}\left[\left(x^{j} y_{j}+x_{j} y^{j}\right) z^{i}+\left(x^{j} z_{j}+x_{j} z^{j}\right) y^{i}+\left(y^{j} z_{j}+y_{j} z^{j}\right) x^{i}\right] \\
& -\frac{1}{6}\left[\left(x^{0} y_{0}+x_{0} y^{0}\right) z^{i}+\left(x^{0} z_{0}+x_{0} z^{0}\right) y^{i}+\left(y^{0} z_{0}+y_{0} z^{0}\right) x^{i}\right] .
\end{aligned}
$$


Let us recall that in this symplectic frame the $2 N \times 2 N$ symplectic metric is given by eq. (6.9). In particular, one can compute the various components of the linear map ${ }^{40}$ $\Upsilon_{x}(y)$ defined in eq. (2.6) to read in components

$$
\begin{aligned}
& \Upsilon_{x}(y)^{0}=-3 T(x, x, y)^{0}+\left(-x^{0} y_{0}-x^{j} y_{j}+x_{0} y^{0}+x_{j} y^{j}\right) x^{0} \\
& \Upsilon_{x}(y)^{i}=-3 T(x, x, y)^{i}+\left(-x^{0} y_{0}-x^{j} y_{j}+x_{0} y^{0}+x_{j} y^{j}\right) x^{i} ; \\
& \Upsilon_{x}(y)_{0}=3 T(x, x, y)_{0}+\left(-x^{0} y_{0}-x^{j} y_{j}+x_{0} y^{0}+x_{j} y^{j}\right) x_{0} ; \\
& \Upsilon_{x}(y)_{i}=3 T(x, x, y)_{i}+\left(-x^{0} y_{0}-x^{j} y_{j}+x_{0} y^{0}+x_{j} y^{j}\right) x_{i},
\end{aligned}
$$

where

$$
\begin{aligned}
T(x, x, y)^{0}= & \frac{1}{3} d_{i j k} x^{i} x^{j} y^{k}-\frac{1}{3}\left[2\left(x^{0}\right)^{2} y_{0}+2 x^{0} x_{0} y^{0}\right]-\frac{1}{3}\left[x^{i} x_{i} y^{0}+\left(x^{i} y_{i}+x_{i} y^{i}\right) x^{0}\right] ; \\
T(x, x, y)^{i}= & -\frac{1}{3} d^{i j k}\left(2 x^{0} x_{j} y_{k}+x_{j} x_{k} y^{0}\right)+\frac{1}{3} d_{k l m} d^{m i j}\left(2 x_{j} x^{k} y^{l}+x^{k} x^{l} y_{j}\right) \\
& -\frac{1}{3}\left[x^{j} x_{j} y^{i}+\left(x^{j} y_{j}+x_{j} y^{j}\right) x^{i}\right]-\frac{1}{3}\left[x^{0} x_{0} y^{i}+\left(x^{0} y_{0}+x_{0} y^{0}\right) x^{i}\right] \\
T(x, x, y)_{0}= & -\frac{1}{3} d^{i j k} x_{i} x_{j} y_{k}-\frac{1}{3}\left[2 x^{0} x_{0} y_{0}+\left(x_{0}\right)^{2} y^{0}\right]-\frac{1}{3}\left[x^{i} x_{i} y_{0}+\left(x^{i} y_{i}+x_{i} y^{i}\right) x_{0}\right] \\
T(x, x, y)_{i}= & \frac{1}{3} d_{i j k}\left(2 x_{0} x^{j} y^{k}+x^{j} x^{k} y_{0}\right)+\frac{1}{3} d_{i j m} d^{m k l}\left(2 x^{j} x_{k} y_{l}+x_{k} x_{l} y^{j}\right) \\
& -\frac{1}{3}\left[x^{j} x_{j} y_{i}+\left(x^{j} y_{j}+x_{j} y^{j}\right) x_{i}\right]-\frac{1}{3}\left[x^{0} x_{0} y_{i}+\left(x^{0} y_{0}+x_{0} y^{0}\right) x_{i}\right] .
\end{aligned}
$$

The following quantity, $x$ - and $y$ - dependent function, plays an important role

$$
\begin{aligned}
\left\{y, \Upsilon_{x}(y)\right\}= & 3\{y, T(x, x, y)\}-\{x, y\}^{2} \\
= & -3\left[-y^{0} T(x, x, y)_{0}-y^{i} T(x, x, y)_{i}+y_{0} T(x, x, y)^{0}+y_{i} T(x, x, y)^{i}\right] \\
& -\left(-x^{0} y_{0}-x^{j} y_{j}+x_{0} y^{0}+x_{j} y^{j}\right)^{2} .
\end{aligned}
$$

where $T(x, x, y)_{0}, T(x, x, y)_{i}, T(x, x, y)^{0}$ and $T(x, x, y)^{i}$ are given above.

Further expressions. Within the $4 D / 5 D$ special coordinates' symplectic frame of reduced FTS's and within the choices eq. (8.52) resp. eq. (8.63) of the rank-4 element $x \in \mathfrak{F}$ (with $\Delta(x)<0$ ) and of the rank-4 element $y \in \mathfrak{F}_{x}^{\perp}=\mathfrak{F} / \mathfrak{F}_{x}$ (with $\Delta(y) \gtrless 0$ ), we present here some further formulas useful for section 8. In order to determine the condition of closure of $\mathfrak{F}_{y \perp x}$ under $T$, we have to explicitly compute $T(r) \equiv T(r, r, r)$ for a generic element $r=a y+b \Upsilon_{x}(y) \in \mathfrak{F}_{y \perp x}$, which is given by

$$
\begin{aligned}
T(r) \equiv & T(r, r, r)=T\left(a y+b \Upsilon_{x}(y), a y+b \Upsilon_{x}(y), a y+b \Upsilon_{x}(y)\right) \\
= & a^{3} T(y, y, y)+3 a^{2} b T\left(y, y, \Upsilon_{x}(y)\right)+3 a^{2} b T\left(\Upsilon_{x}(y), \Upsilon_{x}(y), y\right) \\
& +b^{3} T\left(\Upsilon_{x}(y), \Upsilon_{x}(y), \Upsilon_{x}(y)\right) .
\end{aligned}
$$

\footnotetext{
${ }^{40}$ Note that when $y \in \mathfrak{F}_{x}^{\perp}$, then $\{x, y\}=0$, and the second term in eq. (2.6) is missing [14].
} 
Let us then start and compute the components of $T(r)_{M}$. In first place, the $T(y, y, y)_{M}$ quantities are given by

$$
\begin{aligned}
T(y, y, y)_{0} & =-\frac{1}{3} d^{i j k} y_{i} y_{j} y_{k} ; \\
T(y, y, y)_{i} & =\left(d_{m i j} d^{m k l} y^{j} y_{k} y_{l}-y^{j} y_{j} y_{i}\right) ; \\
T(y, y, y)^{0} & =\frac{1}{3} d_{i j k} y^{i} y^{j} y^{k} ; \\
T(y, y, y)^{i} & =\left(d_{k l m} d^{m i j} y_{j} y^{k} y^{l}-y^{j} y_{j} y^{i}\right) ;
\end{aligned}
$$

similarly

$$
\begin{aligned}
T\left(y, y, \Upsilon_{x}(y)\right)_{0} & =\frac{1}{3} x^{0} x_{0} d^{i j k} y_{i} y_{j} y_{k} \\
T\left(y, y, \Upsilon_{x}(y)\right)_{i} & =-\frac{1}{3} x^{0} x_{0}\left(d_{m i j} d^{m k l} y^{j} y_{k} y_{l}-y^{j} y_{j} y_{i}\right) \\
T\left(y, y, \Upsilon_{x}(y)\right)^{0} & =\frac{1}{3} x^{0} x_{0} d_{i j k} y^{i} y^{j} y^{k} \\
T\left(y, y, \Upsilon_{x}(y)\right)^{i} & =\frac{1}{3} x^{0} x_{0}\left(d_{k l m} d^{m i j} y_{j} y^{k} y^{l}-y^{j} y_{j} y^{i}\right)
\end{aligned}
$$

and

$$
\begin{aligned}
T\left(\Upsilon_{x}(y), \Upsilon_{x}(y), y\right)_{0} & =-\frac{1}{3}\left(x^{0} x_{0}\right)^{2} d^{i j k} y_{i} y_{j} y_{k} ; \\
T\left(\Upsilon_{x}(y), \Upsilon_{x}(y), y\right)_{i} & =-\frac{1}{3}\left(x^{0} x_{0}\right)^{2}\left(d_{m i j} d^{m k l} y^{j} y_{k} y_{l}-y^{j} y_{j} y_{i}\right) \\
T\left(\Upsilon_{x}(y), \Upsilon_{x}(y), y\right)^{0} & =\frac{1}{3}\left(x^{0} x_{0}\right)^{2} d_{i j k} y^{i} y^{j} y^{k} \\
T\left(\Upsilon_{x}(y), \Upsilon_{x}(y), y\right)^{i} & =-\frac{1}{3}\left(x^{0} x_{0}\right)^{2}\left(d_{k l m} d^{m i j} y_{j} y^{k} y^{l}-y^{j} y_{j} y^{i}\right)
\end{aligned}
$$

finally

$$
\begin{aligned}
& T\left(\Upsilon_{x}(y), \Upsilon_{x}(y), \Upsilon_{x}(y)\right)_{0}=\frac{1}{3}\left(x^{0} x_{0}\right)^{3} d^{i j k} y_{i} y_{j} y_{k} \\
& T\left(\Upsilon_{x}(y), \Upsilon_{x}(y), \Upsilon_{x}(y)\right)_{i}=\left(x^{0} x_{0}\right)^{3}\left(d_{m i j} d^{m k l} y^{j} y_{k} y_{l}-y^{j} y_{j} y_{i}\right) \\
& T\left(\Upsilon_{x}(y), \Upsilon_{x}(y), \Upsilon_{x}(y)\right)^{0}=\frac{1}{3}\left(x^{0} x_{0}\right)^{3} d_{i j k} y^{i} y^{j} y^{k} ; \\
& T\left(\Upsilon_{x}(y), \Upsilon_{x}(y), \Upsilon_{x}(y)\right)^{i}=-\left(x^{0} x_{0}\right)^{3}\left(d_{k l m} d^{m i j} y_{j} y^{k} y^{l}-y^{j} y_{j} y^{i}\right) .
\end{aligned}
$$

Therefore, putting together all the computations, the various components of $T(r)_{M}$ reads

$$
\begin{aligned}
T(r)_{0} & =\left(a-x^{0} x_{0} b\right)^{3} T(y, y, y)_{0} ; \\
T(r)_{i} & =\left(a-x^{0} x_{0} b\right)^{2}\left(a+x^{0} x_{0} b\right) T(y, y, y)_{i} ; \\
T(r)^{0} & =\left(a+x^{0} x_{0} b\right)^{3} T(y, y, y)^{0} ; \\
T(r)^{i} & =\left(a-x^{0} x_{0} b\right)\left(a+x^{0} x_{0} b\right)^{2} T(y, y, y)^{i} .
\end{aligned}
$$


Then

$$
T(r)^{M}=\Omega^{M N} T(r)_{N}=\left(-T(r)^{0},-T(r)^{i}, T(r)_{0}, T(r)_{i}\right)^{T},
$$

where $T(r)^{0}, T(r)^{i}, T(r)_{0}$ and $T(r)_{i}$ are given by (8.75). Final expressions are given in the text, see section 8 .

Open Access. This article is distributed under the terms of the Creative Commons Attribution License (CC-BY 4.0), which permits any use, distribution and reproduction in any medium, provided the original author(s) and source are credited.

\section{References}

[1] Event Horizon Telescope collaboration, First M87 Event Horizon Telescope Results. I. The Shadow of the Supermassive Black Hole, Astrophys. J. 875 (2019) L1 [arXiv: 1906.11238] [INSPIRE].

[2] Event Horizon Telescope collaboration, First M87 Event Horizon Telescope Results. VI. The Shadow and Mass of the Central Black Hole, Astrophys. J. 875 (2019) L6 [arXiv: 1906.11243] [INSPIRE].

[3] J.D. Bekenstein, Black holes and the second law, Lett. Nuovo Cim. 379 (1972) 737.

[4] J.D. Bekenstein, Black holes and entropy, Phys. Rev. D 7 (1973) 2333 [InSPIRE].

[5] J.M. Bardeen, B. Carter and S.W. Hawking, The four laws of black hole mechanics, Commun. Math. Phys. 31 (1973) 161 [InSPIRE].

[6] S.W. Hawking, Black hole explosions, Nature 248 (1974) 30 [INSPIRE].

[7] S.W. Hawking, Particle creation by black holes, Commun. Math. Phys. 43 (1975) 199.

[8] A. Strominger and C. Vafa, Microscopic origin of the Bekenstein-Hawking entropy, Phys. Lett. B 379 (1996) 99 [hep-th/9601029] [INSPIRE].

[9] E. Cremmer and B. Julia, The SO(8) Supergravity, Nucl. Phys. B 159 (1979) 141 [inSPIRE].

[10] M.J. Duff and J.X. Lu, Duality Rotations in Membrane Theory, Nucl. Phys. B 347 (1990) 394 [INSPIRE].

[11] C.M. Hull and P.K. Townsend, Unity of superstring dualities, Nucl. Phys. B 438 (1995) 109 [hep-th/9410167] [INSPIRE].

[12] P. Breitenlohner, D. Maison and G.W. Gibbons, Four-Dimensional Black Holes from Kaluza-Klein Theories, Commun. Math. Phys. 120 (1988) 295 [INSPIRE].

[13] S. Ferrara and A. Marrani, Symmetric Spaces in Supergravity, Contemp. Math. 490 (2009) 203 [arXiv:0808.3567] [INSPIRE].

[14] R.B. Brown, Groups of type E7, J. Reine Angew. Math. 236 (1969) 79.

[15] H. Freudenthal, Beziehungen der $E_{7}$ und $E_{8}$ zur oktavenebene I-II, Nederl. Akad. Wetensch. Proc. Ser. 57 (1954) 218.

[16] H. Freudenthal, Beziehungen der $E_{7}$ und $E_{8}$ zur oktavenebene IX, Nederl. Akad. Wetensch. Proc. Ser. A 62 (1959) 466.

[17] L. Borsten, D. Dahanayake, M.J. Duff and W. Rubens, Black holes admitting a Freudenthal dual, Phys. Rev. D 80 (2009) 026003 [arXiv:0903.5517] [InSPIRE]. 
[18] E. Cartan, Eeuvres complètes, editions du Centre National de la Recherche Scientifique, Paris France (1984).

[19] S. Ferrara, A. Marrani and A. Yeranyan, Freudenthal Duality and Generalized Special Geometry, Phys. Lett. B 701 (2011) 640 [arXiv:1102.4857] [InSPIRE].

[20] T. Ortín and C.S. Shahbazi, The Supersymmetric black holes of $N=8$ supergravity, Phys. Rev. D 86 (2012) 061702 [arXiv:1206.3190] [INSPIRE].

[21] P. Galli, P. Meessen and T. Ortín, The Freudenthal gauge symmetry of the black holes of $N=2, d=4$ supergravity, JHEP 05 (2013) 011 [arXiv:1211.7296] [INSPIRE].

[22] L. Borsten, M.J. Duff, S. Ferrara and A. Marrani, Freudenthal Dual Lagrangians, Class. Quant. Grav. 30 (2013) 235003 [arXiv: 1212.3254] [INSPIRE].

[23] M.K. Gaillard and B. Zumino, Duality Rotations for Interacting Fields, Nucl. Phys. B 193 (1981) 221 [INSPIRE].

[24] S. Cecotti, S. Ferrara and L. Girardello, Hidden Noncompact Symmetries in String Theory, Nucl. Phys. B 308 (1988) 436 [inSPIRE].

[25] M.J. Duff, Duality Rotations in String Theory, Nucl. Phys. B 335 (1990) 610 [InSPIRE].

[26] S. Ferrara and R. Kallosh, Creation of Matter in the Universe and Groups of Type E7, JHEP 12 (2011) 096 [arXiv: 1110.4048] [INSPIRE].

[27] S. Ferrara, R. Kallosh and A. Marrani, Degeneration of Groups of Type E7 and Minimal Coupling in Supergravity, JHEP 06 (2012) 074 [arXiv:1202.1290] [INSPIRE].

[28] A. Marrani, C.-X. Qiu, S.-Y.D. Shih, A. Tagliaferro and B. Zumino, Freudenthal Gauge Theory, JHEP 03 (2013) 132 [arXiv:1208.0013] [INSPIRE].

[29] J.J. Fernandez-Melgarejo and E. Torrente-Lujan, $N=2$ SUGRA BPS Multi-center solutions, quadratic prepotentials and Freudenthal transformations, JHEP 05 (2014) 081 [arXiv: 1310.4182] [INSPIRE].

[30] L. Borsten, M.J. Duff and A. Marrani, Freudenthal duality and conformal isometries of extremal black holes, arXiv:1812.10076 [INSPIRE].

[31] L. Borsten, D. Dahanayake, M.J. Duff, W. Rubens and H. Ebrahim, Wrapped branes as qubits, Phys. Rev. Lett. 100 (2008) 251602 [arXiv:0802.0840] [INSPIRE].

[32] P. Levay, M. Saniga and P. Vrana, Three-Qubit Operators, the Split Cayley Hexagon of Order Two and Black Holes, Phys. Rev. D 78 (2008) 124022 [arXiv:0808.3849] [INSPIRE].

[33] L. Borsten, D. Dahanayake, M.J. Duff, W. Rubens and H. Ebrahim, Freudenthal triple classification of three-qubit entanglement, Phys. Rev. A 80 (2009) 032326 [arXiv: 0812.3322] [INSPIRE].

[34] P. Levay and G. Sarosi, Hitchin functionals are related to measures of entanglement, Phys. Rev. D 86 (2012) 105038 [arXiv:1206.5066] [INSPIRE].

[35] A. Ashmore, Marginal deformations of $3 d \mathcal{N}=2$ CFTs from AdS $S_{4}$ backgrounds in generalised geometry, JHEP 12 (2018) 060 [arXiv:1809.03503] [INSPIRE].

[36] L. Borsten, D. Dahanayake, M.J. Duff, H. Ebrahim and W. Rubens, Black Holes, Qubits and Octonions, Phys. Rept. 471 (2009) 113 [arXiv:0809.4685] [INSPIRE].

[37] L. Borsten, D. Dahanayake, M.J. Duff, A. Marrani and W. Rubens, Four-qubit entanglement from string theory, Phys. Rev. Lett. 105 (2010) 100507 [arXiv:1005.4915] [INSPIRE]. 
[38] L. Borsten, M.J. Duff, A. Marrani and W. Rubens, On the Black-Hole/Qubit Correspondence, Eur. Phys. J. Plus 126 (2011) 37 [arXiv:1101.3559] [InSPIRE].

[39] L. Borsten, M.J. Duff and P. Levay, The black-hole/qubit correspondence: an up-to-date review, Class. Quant. Grav. 29 (2012) 224008 [arXiv:1206.3166] [INSPIRE].

[40] P. Jordan, J. von Neumann and E.P. Wigner, On an Algebraic generalization of the quantum mechanical formalism, Annals Math. 35 (1934) 29 [INSPIRE].

[41] K. Meyberg, Eine theorie der freudenthalschen tripelsysteme. i, ii, Nederl. Akad. Wetensch. Proc. Ser. A 71 (1968) 162.

[42] M. Günaydin, G. Sierra and P.K. Townsend, Exceptional Supergravity Theories and the MAGIC Square, Phys. Lett. 133B (1983) 72 [INSPIRE].

[43] M. Günaydin, G. Sierra and P.K. Townsend, The Geometry of $N=2$ Maxwell-Einstein Supergravity and Jordan Algebras, Nucl. Phys. B 242 (1984) 244 [InSPIRE].

[44] S. Ferrara and M. Günaydin, Orbits of exceptional groups, duality and BPS states in string theory, Int. J. Mod. Phys. A 13 (1998) 2075 [hep-th/9708025] [InSPIRE].

[45] L. Borsten, M.J. Duff, S. Ferrara, A. Marrani and W. Rubens, Explicit Orbit Classification of Reducible Jordan Algebras and Freudenthal Triple Systems, Commun. Math. Phys. 325 (2014) 17 [arXiv: 1108.0908] [inSPIRE].

[46] S. Krutelevich, Jordan algebras, exceptional groups, and Bhargava composition, J. Algebra 314 (2007) 924 [math/0411104].

[47] I. Yokota, Exceptional Lie groups, arXiv:0902.0431.

[48] L. Borsten, D. Dahanayake, M.J. Duff and W. Rubens, Black holes admitting a Freudenthal dual, Phys. Rev. D 80 (2009) 026003 [arXiv:0903.5517] [InSPIRE].

[49] C.J. Ferrar, Strictly Regular Elements in Freudenthal Triple Systems, Trans. Am. Math. Soc. 174 (1972) 313.

[50] L. Borsten, M.J. Duff, S. Ferrara, A. Marrani and W. Rubens, Explicit Orbit Classification of Reducible Jordan Algebras and Freudenthal Triple Systems, Commun. Math. Phys. 325 (2014) 17 [arXiv: 1108.0908] [INSPIRE].

[51] E. Cremmer and B. Julia, The $\mathcal{N}=8$ Supergravity Theory. 1. The Lagrangian, Phys. Lett. B 80 (1978) 48.

[52] E. Cremmer and B. Julia, The SO(8) Supergravity, Nucl. Phys. B 159 (1979) 141 [inSPIRE].

[53] C.M. Hull and P.K. Townsend, Unity of superstring dualities, Nucl. Phys. B 438 (1995) 109 [hep-th/9410167] [INSPIRE].

[54] M. Günaydin and O. Pavlyk, Quasiconformal Realizations of $E(6)(6), E(7)(7), E(8)(8)$ and $S O(n+3, m+3), N \geq 4$ Supergravity and Spherical Vectors, Adv. Theor. Math. Phys. 13 (2009) 1895 [INSPIRE].

[55] M. Günaydin, S. McReynolds and M. Zagermann, Unified $N=2$ Maxwell-Einstein and Yang-Mills-Einstein supergravity theories in four dimensions, JHEP 09 (2005) 026 [hep-th/0507227] [INSPIRE].

[56] S.L. Cacciatori, B.L. Cerchiai and A. Marrani, Squaring the Magic, Adv. Theor. Math. Phys. 19 (2015) 923 [arXiv:1208.6153] [inSPIRE]. 
[57] A. Marrani, G. Pradisi, F. Riccioni and L. Romano, Nonsupersymmetric magic theories and Ehlers truncations, Int. J. Mod. Phys. A 32 (2017) 1750120 [arXiv:1701.03031] [InSPIRE].

[58] E. Torrente-Lujan, Black Holes and cuadratic Freudhental triple systems, to appear.

[59] L. Borsten, M.J. Duff, S. Ferrara, A. Marrani and W. Rubens, Small Orbits, Phys. Rev. D 85 (2012) 086002 [arXiv: 1108.0424] [INSPIRE].

[60] A. Ceresole, S. Ferrara, A. Gnecchi and A. Marrani, More on $N=8$ Attractors, Phys. Rev. D 80 (2009) 045020 [arXiv:0904.4506] [InSPIRE].

[61] J.F. Luciani, Coupling of O(2) Supergravity with Several Vector Multiplets, Nucl. Phys. B 132 (1978) 325 [INSPIRE].

[62] S. Ferrara, A. Gnecchi and A. Marrani, $d=4$ Attractors, Effective Horizon Radius and Fake Supergravity, Phys. Rev. D 78 (2008) 065003 [arXiv:0806.3196] [INSPIRE].

[63] S. Ferrara, A. Marrani and E. Orazi, Split Attractor Flow in $N=2$ Minimally Coupled Supergravity, Nucl. Phys. B 846 (2011) 512 [arXiv:1010.2280] [INSPIRE].

[64] R.S. Garibaldi, Groups of type E F $_{7}$ over Arbitrary Fields, Commun. Algebra 29 (2001) 2689 [math/9811056].

[65] L. Castellani, A. Ceresole, S. Ferrara, R. D'Auria, P. Fré and E. Maina, The Complete $N=3$ Matter Coupled Supergravity, Nucl. Phys. B 268 (1986) 317 [InSPIRE].

[66] E. Cremmer, J. Scherk and S. Ferrara, SU(4) Invariant Supergravity Theory, Phys. Lett. B 74 (1978) 61 [INSPIRE].

[67] B. de Wit and H. Nicolai, Extended Supergravity With Local SO(5) Invariance, Nucl. Phys. B 188 (1981) 98 [INSPIRE].

[68] A. Marrani, E. Orazi and F. Riccioni, Exceptional Reductions, J. Phys. A 44 (2011) 155207 [arXiv: 1012.5797] [INSPIRE].

[69] L. Andrianopoli, R. D'Auria, S. Ferrara, A. Marrani and M. Trigiante, Two-Centered Magical Charge Orbits, JHEP 04 (2011) 041 [arXiv:1101.3496] [INSPIRE].

[70] S. Ferrara, K. Hayakawa and A. Marrani, Lectures on Attractors and Black Holes, Fortsch. Phys. 56 (2008) 993 [arXiv:0805.2498] [INSPIRE].

[71] V.G. Kac, Some Remarks on Nilpotent Orbits, J. Algebra 64 (1980) 190.

[72] M. Sato and T. Kimura, A classification of irreducible prehomogeneous vector spaces and their relative invariants, Nagoya Math. J. 65 (1977) 1.

[73] A. Ceresole, S. Ferrara and A. Marrani, 4d/5d Correspondence for the Black Hole Potential and its Critical Points, Class. Quant. Grav. 24 (2007) 5651 [arXiv:0707.0964] [INSPIRE].

[74] K. McCrimmon, A Taste of Jordan Algebras, Springer-Verlag, New York U.S.A. (2004).

[75] L. Borsten, M.J. Duff, S. Ferrara, A. Marrani and W. Rubens, Small Orbits, Phys. Rev. D 85 (2012) 086002 [arXiv: 1108.0424] [INSPIRE].

[76] O. Shukuzawa, Explicit classifications of orbits in Jordan algebra and Freudenthal vector space over the exceptional Lie groups, Commun. Algebra 34 (2006) 197.

[77] S. Ferrara and O. Maciá, Real symplectic formulation of local special geometry, Phys. Lett. B 637 (2006) 102 [hep-th/0603111] [INSPIRE]. 
[78] T. Mohaupt and O. Vaughan, The Hesse potential, the c-map and black hole solutions, JHEP 07 (2012) 163 [arXiv: 1112.2876] [INSPIRE].

[79] D. Klemm and O. Vaughan, Nonextremal black holes in gauged supergravity and the real formulation of special geometry, JHEP 01 (2013) 053 [arXiv:1207.2679] [INSPIRE].

[80] S. Bellucci, S. Ferrara, A. Marrani and A. Yeranyan, stu Black Holes Unveiled, Entropy 10 (2008) 507 [arXiv:0807.3503] [INSPIRE].

[81] A. Sen and C. Vafa, Dual pairs of type-II string compactification, Nucl. Phys. B 455 (1995) 165 [hep-th/9508064] [INSPIRE].

[82] M.J. Duff, J.T. Liu and J. Rahmfeld, Four-dimensional string-string-string triality, Nucl. Phys. B 459 (1996) 125 [hep-th/9508094] [INSPIRE].

[83] K. Behrndt, R. Kallosh, J. Rahmfeld, M. Shmakova and W.K. Wong, STU black holes and string triality, Phys. Rev. D 54 (1996) 6293 [hep-th/9608059] [INSPIRE].

[84] E. Cremmer et al., Vector Multiplets Coupled to $N=2$ Supergravity: SuperHiggs Effect, Flat Potentials and Geometric Structure, Nucl. Phys. B 250 (1985) 385 [inSPIRE].

[85] S. Ferrara, A. Marrani, E. Orazi and M. Trigiante, Dualities Near the Horizon, JHEP 11 (2013) 056 [arXiv: 1305.2057] [INSPIRE].

[86] L. Andrianopoli, R. D'Auria and S. Ferrara, U-duality and central charges in various dimensions revisited, Int. J. Mod. Phys. A 13 (1998) 431 [hep-th/9612105] [INSPIRE].

[87] P. Aschieri, S. Ferrara and B. Zumino, Duality Rotations in Nonlinear Electrodynamics and in Extended Supergravity, Riv. Nuovo Cim. 31 (2008) 625 [arXiv:0807.4039] [INSPIRE].

[88] S. Murakami, On the Automorphisms of a Real Semi-simple Lie Algebra, J. Math. Soc. Jap. 4 (1952) 103.

[89] M. Trigiante, Gauged Supergravities, Phys. Rept. 680 (2017) 1 [arXiv:1609.09745] [INSPIRE].

[90] A. Gallerati and M. Trigiante, Introductory Lectures on Extended Supergravities and Gaugings, Springer Proc. Phys. 176 (2016) 41 [arXiv: 1809.10647] [INSPIRE].

[91] R. Kallosh and A.D. Linde, Strings, black holes and quantum information, Phys. Rev. D 73 (2006) 104033 [hep-th/0602061] [INSPIRE].

[92] E.G. Gimon, F. Larsen and J. Simon, Black holes in Supergravity: The Non-BPS branch, JHEP 01 (2008) 040 [arXiv:0710.4967] [InSPIRE].

[93] G.B. Seligman, On the split exceptional Lie algebra E7, dittoed notes, Yale University, New Haven U.S.A. (1962).

[94] E. Torrente-Lujan, Black Holes in 5D and general Jordan dualities, to appear.

[95] S. Ferrara, A. Marrani and A. Yeranyan, Freudenthal Duality and Generalized Special Geometry, Phys. Lett. B 701 (2011) 640 [arXiv:1102.4857] [InSPIRE].

[96] G. Bossard and C. Ruef, Interacting non-BPS black holes, Gen. Rel. Grav. 44 (2012) 21 [arXiv: 1106.5806] [INSPIRE].

[97] D. Cassani, S. Ferrara, A. Marrani, J.F. Morales and H. Samtleben, A Special road to AdS vacua, JHEP 02 (2010) 027 [arXiv:0911.2708] [INSPIRE].

[98] A. Ceresole, R. D'Auria and S. Ferrara, The Symplectic structure of $N=2$ supergravity and its central extension, Nucl. Phys. Proc. Suppl. 46 (1996) 67 [hep-th/9509160] [InSPIRE]. 
[99] S. Ferrara and R. Kallosh, On $N=8$ attractors, Phys. Rev. D 73 (2006) 125005 [hep-th/0603247] [INSPIRE].

[100] S. Ferrara, R. Kallosh and A. Strominger, $N=2$ extremal black holes, Phys. Rev. D 52 (1995) R5412 [hep-th/9508072] [INSPIRE].

[101] A. Strominger, Macroscopic entropy of $N=2$ extremal black holes, Phys. Lett. B 383 (1996) 39 [hep-th/9602111] [INSPIRE].

[102] S. Ferrara and R. Kallosh, Supersymmetry and attractors, Phys. Rev. D 54 (1996) 1514 [hep-th/9602136] [INSPIRE].

[103] S. Ferrara and R. Kallosh, Universality of supersymmetric attractors, Phys. Rev. D 54 (1996) 1525 [hep-th/9603090] [INSPIRE].

[104] S. Ferrara, G.W. Gibbons and R. Kallosh, Black holes and critical points in moduli space, Nucl. Phys. B 500 (1997) 75 [hep-th/9702103] [inSPIRE]. 